\title{
Investigation of Bartonella quintana Host Immune Evasion
}

\section{Callum Lambert}

A thesis submitted to the Victoria University of Wellington in fulfilment for the degree of Master of Science in Molecular Microbiology

Victoria University of Wellington

2018 



\section{Abstract}

Bartonella is a genus of gram-negative alphaproteobacteria that infect mammals, causing both acute and chronic disease. Bartonella are re-emerging infectious pathogens that cause a variety of clinical syndromes in humans worldwide, including cat scratch disease, trench fever, bacillary angiomatosis, and endocarditis. Bartonella spp. are spread by biting arthropods such as the sand fly, cat flea, and body louse, and have been isolated from almost all mammalian species tested. Bartonella are a re-emerging concern as the number of confirmed Bartonella diagnoses are increasing, primarily in immunocompromised groups, homeless populations, refugee camps, and in veterinary workers. The three primary human disease-causing Bartonella spp. are B. henselae, B. quintana, and B. bacilliformis. Bartonella are known to subvert the host immune system and persist within the host, often causing bacteraemia which is difficult to effectively diagnose and treat. B. quintana infects humans; after introduction to the skin the bacteria implement numerous immune evasion mechanisms to enter the bloodstream and invade erythrocytes. The mechanisms by which $B$. quintana modulates and evades the immune system during early infection are almost entirely unknown. Following exposure to B. quintana, the bacteria encounter host immune cells but survive, evading these cells and disseminating into the lymphatic system and eventually bloodstream. This thesis project aimed to dissect the interactions between B. quintana and the human innate immune system to better understand the early stages of infection. A gentamicin protection assay was developed to investigate the ability of THP-1 macrophages, representing human macrophages present in the skin, to internalise $B$. quintana. These data revealed THP-1 cells were unable to effectively internalise B. quintana, although the mechanism responsible was not determined. Subsequent experiments investigated the role of the B. quintana Type IV secreted effector protein BepA1 in the inhibition of internalisation. Bacterial effector proteins often pathogenically modulate host cell signalling to benefit the bacteria, i.e., altering the actin cytoskeleton to inhibit phagocytosis or supressing immune responses. It was hypothesised BepA1 could play a role in inhibiting phagocytosis; therefore, the host cell target of BepA1 was investigated with a yeast two-hybrid system assay. The human protein Myozap was uncovered as a potential protein that interacts with BepA1. Myozap is expressed in cardiac and lung tissue as well as epithelial and endothelial cells, where it modulates Rho-dependent actin signalling, potentially affecting the actin cytoskeleton and the transcription factor MRTF-A, which influences immune reaction through modulation of NF- $\kappa$ B. To investigate the functional effects of BepA1 activity in host cells, HeLa cells were transfected with BepA1; cell migration and cytokine secretion were assessed, revealing a 
decrease in pro-inflammatory cytokines in BepA1-transfected cells in response to TNF- $\alpha$ stimulation. These data suggest BepA1 may be deployed by B. quintana during infection to suppress the host immune response and avoid clearance from the site of infection. This research addressed a major gap in our understanding of $B$. quintana infections. Improving our understanding of the interactions between Bartonella and the host immune system is an essential first step in the development of improved diagnostic techniques and treatments. 


\section{Acknowledgements}

Firstly, a huge thank you to my supervisor Dr. Joanna MacKichan. It's hard to describe how much I appreciate everything you've done for me. Your undergraduate lectures cultured my love for microbiology and imbued me with passion for the field. Thank you deeply for welcoming me into your lab and finding me a project, the guidance and support during my research has been invaluable and I really appreciate all your help. Your effort in reviewing drafts and giving feedback is amazing and it really does show how much you want your students to succeed. You're the best supervisor out there, thank you so much.

Alvey, these years wouldn't have been the same without you. Our teamwork and efficiency in the lab is second to none and your words of comfort when nothing seemed to be working really did keep me motivated to push on. The famous "everything is fine" quote was often enough, and our nonsensical form of communication really does demonstrate how language evolves. Whether it was taking breaks, troubleshooting when things went wrong, celebrating when things were working, or playing nerd games, I really appreciate your company. Our friendship is one of the most valuable things I've gained during my postgraduate life.

Vimal thank you for all your help in the lab, ELISAs, flow, letting me talk my problems at you. You give a lot of your time helping plebs like me and it's appreciated. Your friendship and entertainment in the lab helped me a lot when faced with writing. Big thank you to Rose, Remy, and Matt for all your encouragement and help. Thank you to everyone else in the AM office for making an awesome and friendly environment. Thank you ChemGen for all the help with my uncooperative yeast, you the real MVP.

Dana, thank you for letting me complain at you all the time, hanging out, being the coolest. Our joint desire to get swol gets me out of bed in the morning (even if it's at a time no human should roam the earth). Our time together reminds me of a quote from the great philosopher, Arnie "let's get serious"; wise words which really drove me to succeed. To my family, particularly Brianna and Danielle, thank you for all your support and help; you lessened the strain during difficult times and that's greatly appreciated. Steven thank you for your friendship, as always, and for giving me plenty of reasons to keep chirpy. To all my other friends and family who supported me during my Masters, thank you for everything! 


\section{Table of Contents}

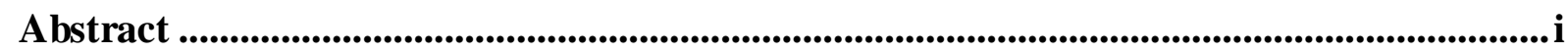

Acknowledgements ........................................................................................................................iii

Table of Contents......................................................................................................................iv

List of Figures ....................................................................................................................................... vi

List of Tables..................................................................................................................................... vi

List of Appendix Figures.......................................................................................................................vii

Abbre viations ......................................................................................................................................... viii

Chapter 1 Introduction .............................................................................................................1

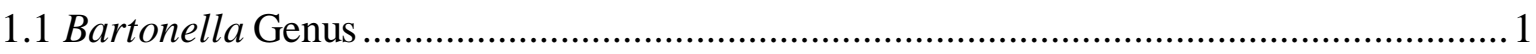

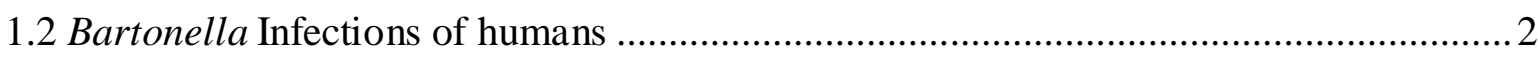

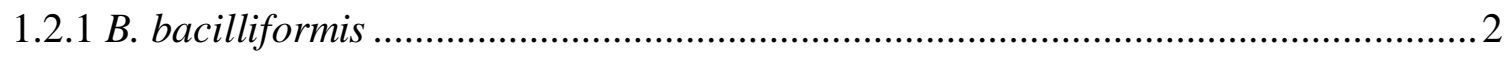

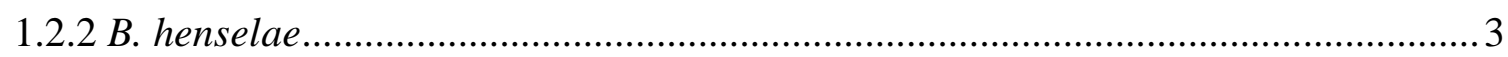

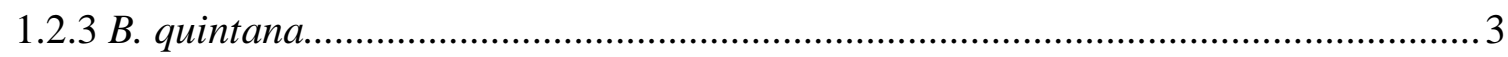

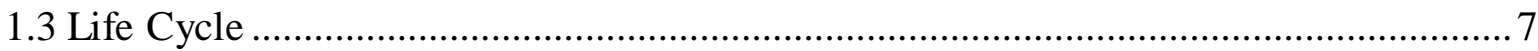

1.4 Immunology \& Immune Evasion by Bartonella ........................................................ 8

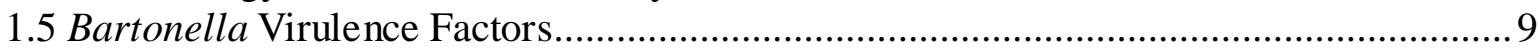

1.6 Role of Bartonella Type IV secretion system in Virulence ........................................ 10

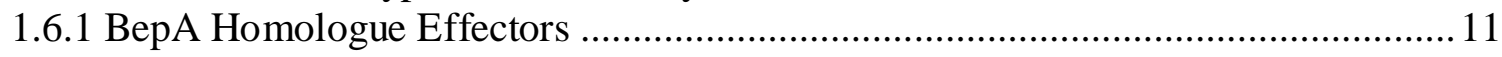

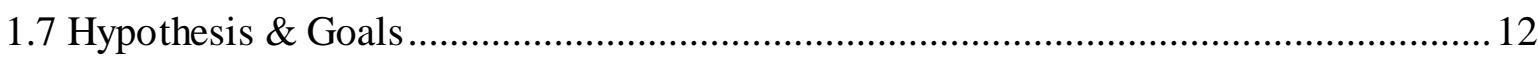

Chapter 2 Methods .....................................................................................................................14

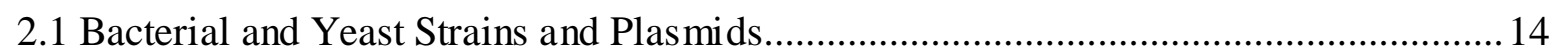

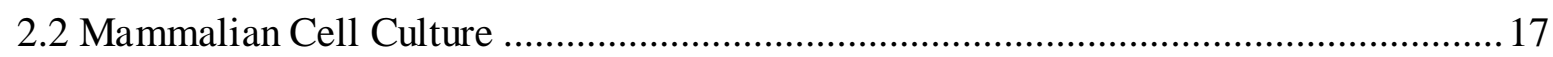

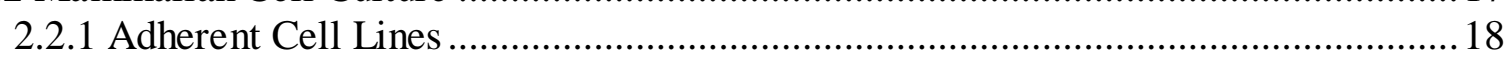

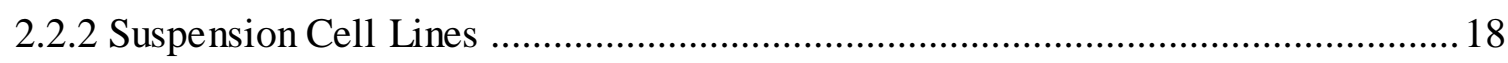

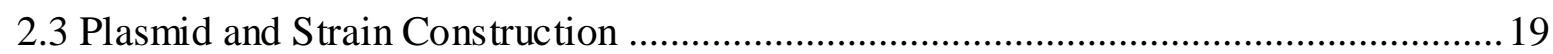

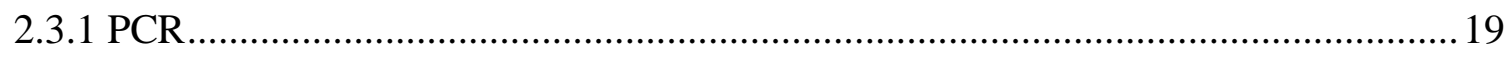

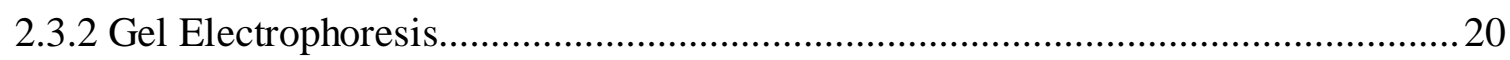

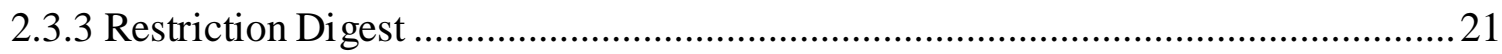

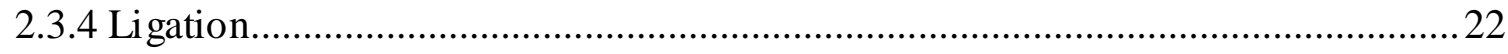

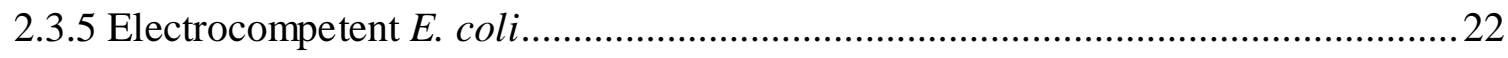

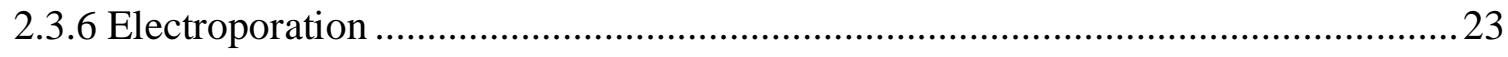

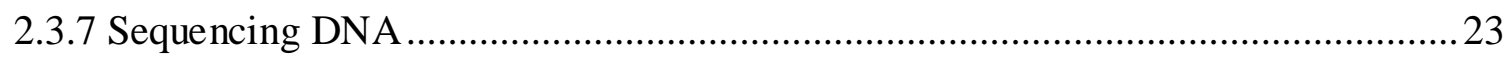

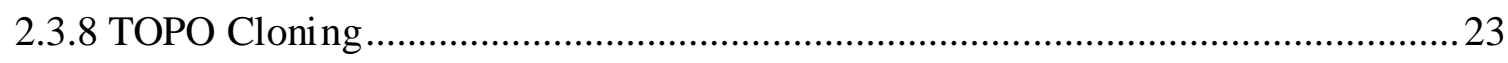

2.4 Bartonella Phagocytosis and Intracellular Survival....................................................24

2.4.1 Gentamicin Protection Assay of Adherent Phagoc ytes ........................................2 24

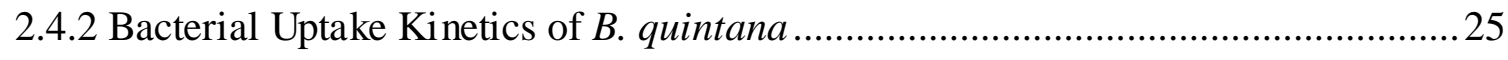

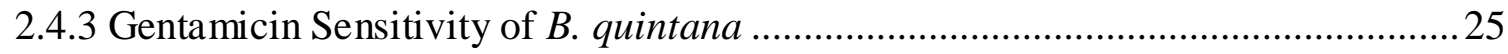

2.5 Yeast Two-Hybrid Screen for Host Cell Targets of BepA1 ........................................25

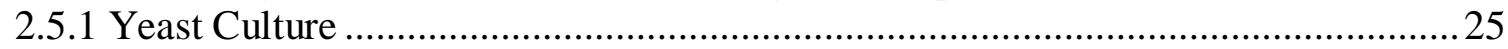




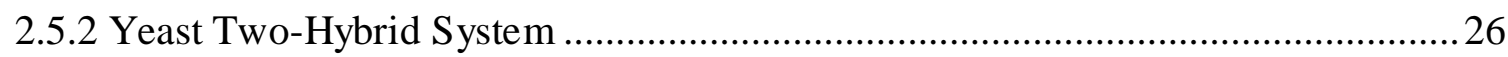

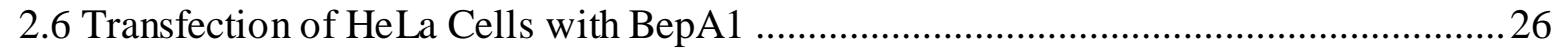

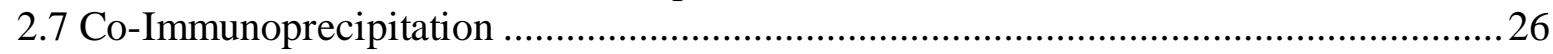

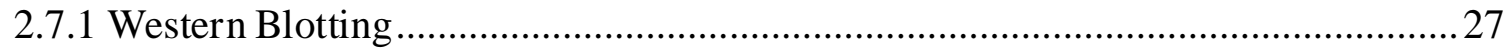

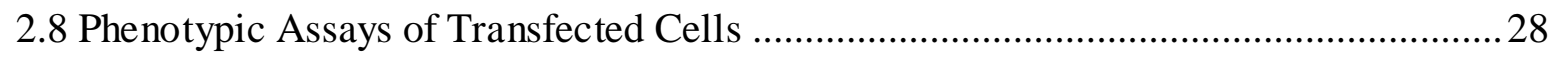

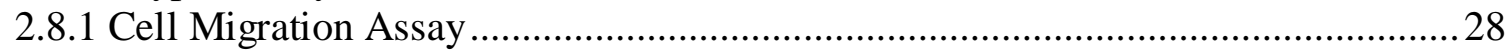

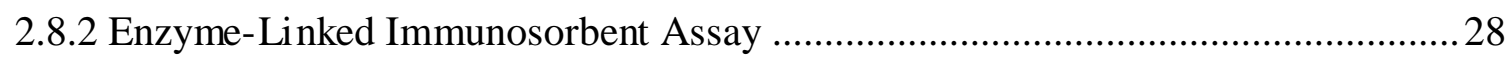

Chapter 3: Interactions Between Bartonella and Macrophages .....................................29

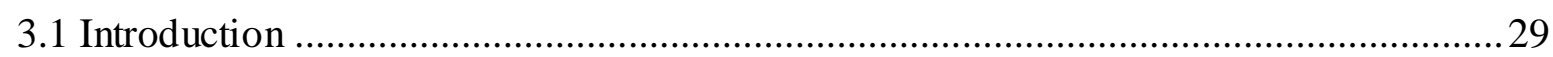

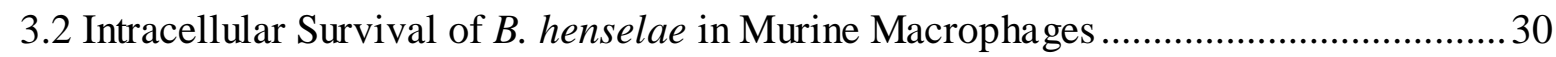

3.3 Intracellular Survival of $B$. henselae in Human Macrophages ..................................... 32

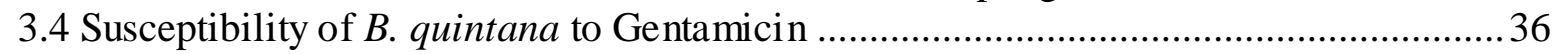

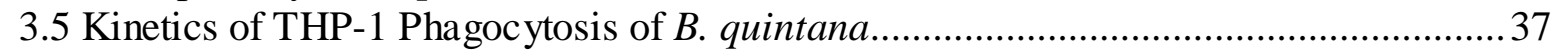

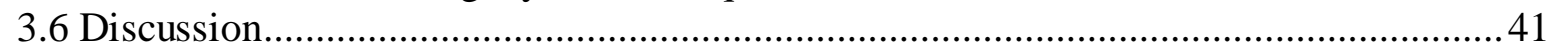

Chapter 4: BepA1 Protein Interaction Studies .................................................................................45

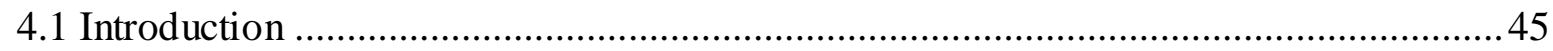

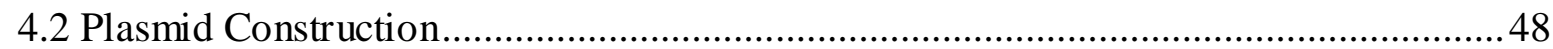

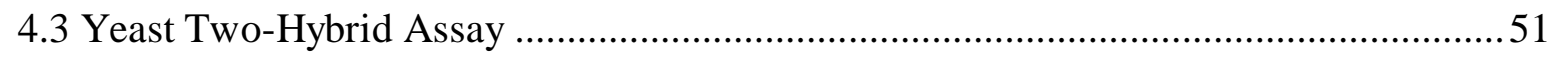

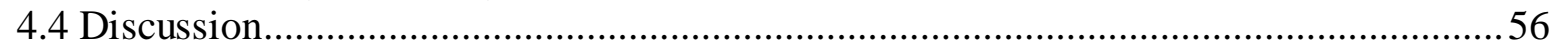

Chapter 5: Functional Assays of BepA1 Transfected Cells ................................................60

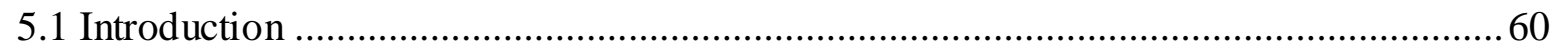

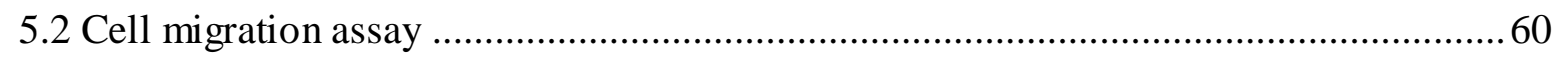

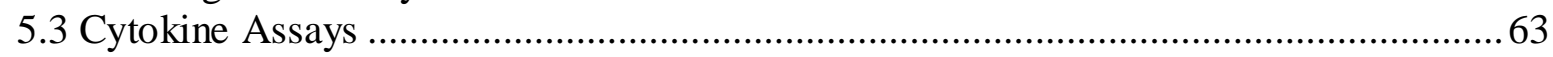

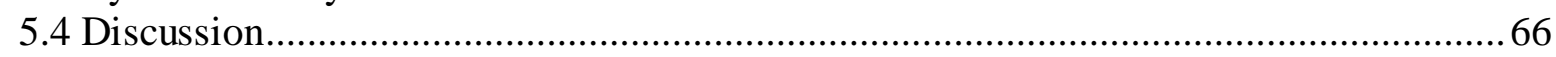

Chapter 6 Research Motivations, Key Findings, and Fut ure Directions ............................70

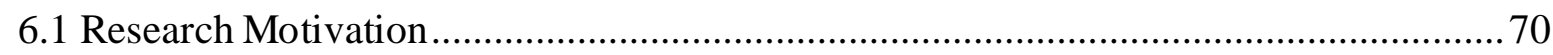

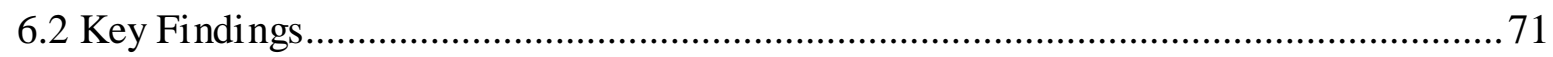

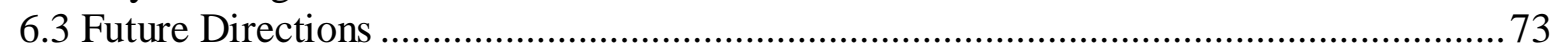

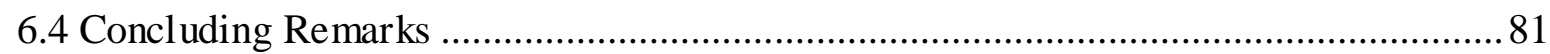

References....................................................................................................................................................8 82

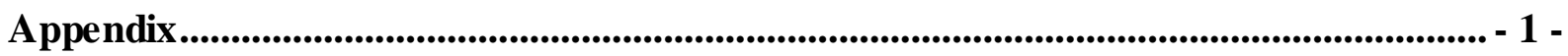




\section{List of Figures}

Figure 3-1: Intracellular B. henselae are killed in RAW 267.4 macrophages .........................31

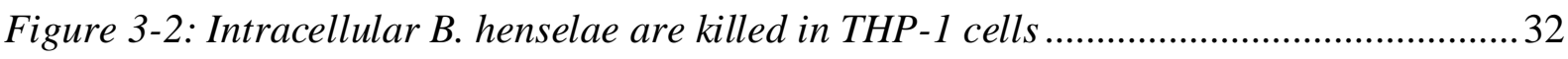

Figure 3-3: Trends in B. henselae killing by RAW 267.4 cells and THP-1s ..........................33

Figure 3-4: Trends in B. henselae killing by RAW 267.4 cells and THP-1 cells on a semi-

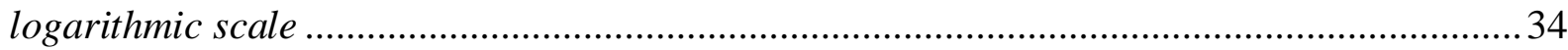

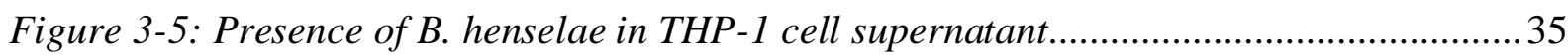

Figure 3-6: B. quintana recovered after exposure gentamicin .............................................37

Figure 3-7: B. quintana isolated from THP-1 cells, with varying co-incubation times............38

Figure 4-1: Bartonella Effector genes are semi-conserved between B. henselae and B. quintana

Figure 4-2: BepA1 and BepA2 inserts following PCR …...................................................49

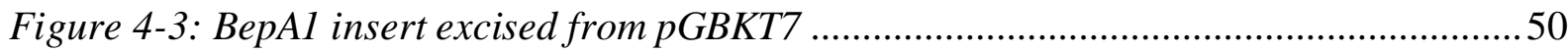

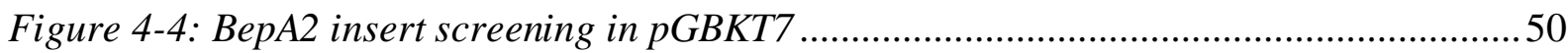

Figure 4-5: Immunoblot of anti-Myozap precipitated proteins; BepAl was not detected in the

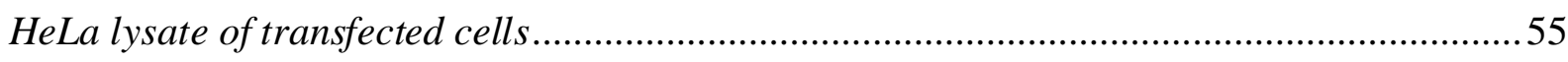

Figure 5-1: Wound Repair in HeLa cells Transfected with BepAl over 22 hours ..................61

Figure 5-2: Cell migration assay showing no significant difference between BepAl transfected HeLa cells and untransfected. .62

Figure 5-3: IL-6 ELISA displaying a significant difference between BepAl transfected HeLa cells and controls........

Figure 5-4: IL-8 ELISA displaying a significant difference between BepA-transfected HeLa cells and controls.

Figure 5-5: Possible signaling pathways affected by BepAl, resulting in inhibition of cytokine secretion 66

\section{List of Tables}

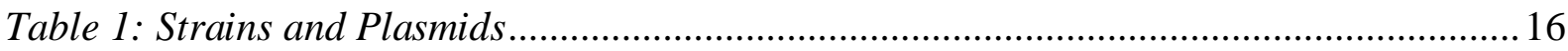

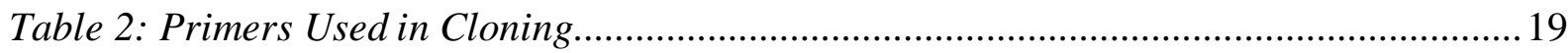

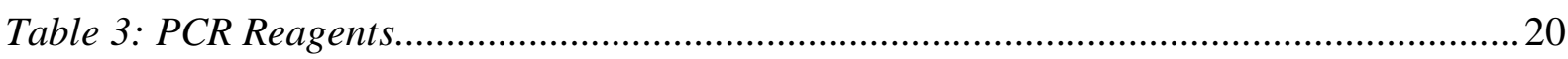

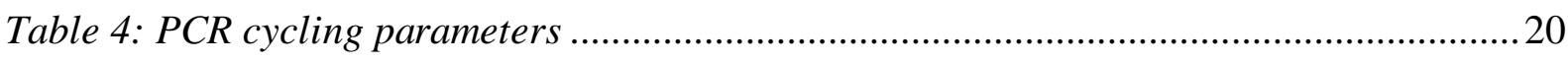

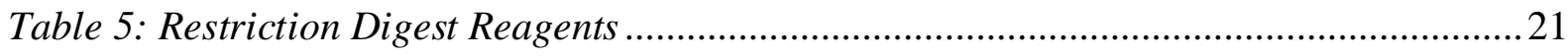

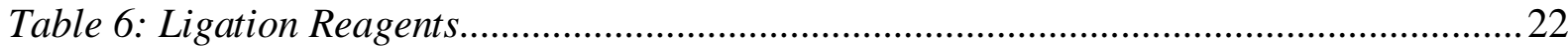




\section{List of Appendix Figures}

Appendix Figure 1: RAW 267.4 cell killing of intracellular B. henselae with a logarithmic $y$ axis. $-1-$

Appendix Figure 2: B. quintana recovered after gentamicin exposure, includes outlier...... - 2 Appendix Figure 3: Homology between B. henselae BepA and B. quintana BepAl/2 ......... - 3 Appendix Figure 4: bepAl insert into pGBKT7 ............................................................. - 3 -

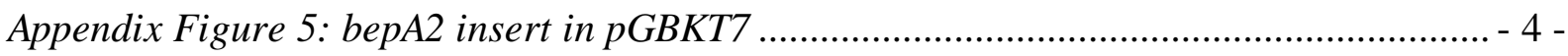
Appendix Figure 6: bepA2 in pGBKT7, Mutated Start Codon........................................... - 4 Appendix Figure 7: BepAl auto-activation test ................................................................. - 5 -

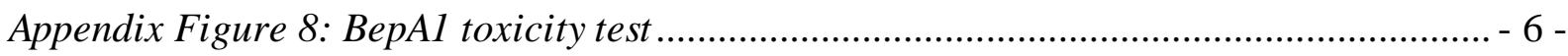
Appendix Figure 9: BLAST match of Y2HS prey sequence - HAS2 antisense RNA 1 \# 1 ..... - 7 Appendix Figure 10: BLAST match of Y2HS prey sequence - HAS2 antisense RNA 1 \#2 ... - 8 Appendix Figure 11: BLAST match of Y2HS prey sequence - HAS2 antisense RNA 1 \#3 ... - 9 Appendix Figure 12: BLAST match of Y2HS prey sequence - HAS2 antisense RNA 1 \#4 . - 10 Appendix Figure 13: BLAST match of Y2HS prey sequence - Myozap protein \#1............. - 11 Appendix Figure 14: BLAST match of Y2HS prey sequence - Myozap protein \#2............. - 12 - 


\section{Abbreviations}

Bep - Bartonella effector protein

BepA1/2 - BepA1 and BepA2 proteins

CBA - Columbia Blood Agar

CFU - Colony Forming Unit

CSD - Cat Scratch Disease

DMSO - Dimethyl sulfoxide

EDTA - Ethylenediaminetetraacetic acid

FCS - Foetal Calf Serum

$\mathrm{IL}$ - Interleukin

LPS - Lipopolysaccharide

MOI - Multiplicity of Infection

$\mathrm{NF}-\kappa \mathrm{B}$ - nuclear factor kappa-light-chain-enhancer of activated B cells

PAMPs - Pathogen-associated molecular patterns

PMA - Phorbol 12-myristate 13-acetate

SDS - sodium dodecyl sulfate

T4SS - Type IV Secretion System

TAE - Tris/acetic acid/EDTA

TNF- $\alpha$ - Tumour necrosis factor alpha

Tris - Tris(hydroxymethyl)ami nomethane

TSB - Tryptic soy broth 


\section{Chapter 1 Introduction}

\subsection{Bartonella Genus}

Emerging and re-emerging infectious diseases place a significant burden on society, having a tremendous impact on both public health and global economics ${ }^{1}$. A 2008 study showed that emerging infectious diseases have risen significantly over the last century ${ }^{1}$. Approximately $60 \%$ of emerging infectious diseases are attributed to zoonotic pathogens, which are spread by animals; $53.4 \%$ of these new zoonotic diseases were caused by bacteria ${ }^{1}$. The Bartonella genus is one of these emerging zoonotic pathogens ${ }^{2}$; seventeen Bartonella species are known to cause human disease,${ }^{3}$ highlighting the importance of improved understanding. Bartonella is a genus of rod shaped, gram-negative alphaproteobacteria that infect mammals and cause a variety of clinical syndromes in humans worldwide, both acute and chronic ${ }^{4,5}$. Bartonella spp. are spread by biting arthropods such as the sand fly, cat flea, and body louse. Bartonella spp. have been isolated from almost all mammalian species tested, including kangaroos and marine mammals, such as sea otters, indicating the extensive range of Bartonella ${ }^{6,7}$. Each Bartonella species typically infects a single or a few closely related mammalian species, known as their reservoir host, but are also capable of causing disease in other mammalian hosts, referred to as incidental hosts $^{8,9}$. In reservoir host infection the bacteria invade and reside in red blood cells, facilitating transmission to a new host via an arthropod blood meal. In incidental hosts, although the infection can be serious, transmission is unlikely as the bacteria do not invade red blood cells. Zoonotic, incidental Bartonella infections are typically self-limiting in immunocompetent individuals; however, in rare cases serious chronic illness can result. Bartonella are a reemerging concern as the number of diagnoses are increasing. Diagnosis of Bartonella infection is difficult and treatment strategies are not well developed. Bartonella species infect using a unique strategy, subverting the host immune response to persist for months or years in the host bloodstream. Bartonella achieve chronic infection through numerous immune evasion mechanisms. For example, in reservoir host infection Bartonella spp. invade erythrocytes, persisting intracellularly, hidden from immune detection. The symptoms of Bartonella infection, referred to as Bartonellosis, can be non-specific in immunocompetent hosts making diagnosis difficult ${ }^{8,10}$. As Bartonella has been isolated from a staggering number of mammalian species, veterinary workers and other professions that have high exposure to mammals are recognised as a high-risk group for incidental infection. 
Bartonella spp. are grouped into evolutionary lineages, although these lineages are constantly improved as known and novel Bartonella genomes are sequenced, improving the accuracy of the Bartonella phylogenetic tree. B. australis, isolated from kangaroos, is the earliest known diverging species and does not currently fall within a lineage group ${ }^{11}$. Group A contains $B$. bacilliformis $^{12}$, among other Bartonella spp, which infects humans as the reservoir host. ${ }^{11}$. Bartonella spp. of Group B infect cattle or deer as their reservoir hosts. Group C is the largest group, including B. henselae and B. quintana. Bartonella spp. of Group C infect voles, dogs, rabbits, mice, rats, cats, or humans as their reservoir hosts ${ }^{12}$. The three primary human disease causing Bartonella spp. are B. henselae, B. quintana, and B. bacilliformis. Humans are an incidental host for B. henselae, but the reservoir host for B. quintana and B. bacilliformis infections.

\subsection{Bartonella Infections of Humans}

\subsubsection{B. bacilliformis}

Humans are the only known reservoir host for B. bacilliformis infection, a disease given many names: bartonellosis, Carrión's disease, Oroya fever, and verruga peruana ${ }^{13}$. In 1885 Daniel Alcides Carrión determined the chronic disease, which presents as warts, was linked to acute Oroya fever ${ }^{14}$. Unfortunately, he came to this discovery as he had a friend inoculate him with fluid from the warts; he subsequently contracted the haemolytic disease and died. After Carrión's death the disease became known as Carrión's disease, recognising the sacrifice of his experimentation. In 1905 B. bacilliformis was identified as the causative agent of Carrión's disease by Dr Alberto Barton. This was the first disease-causing Bartonella discovered; Dr Barton named the disease bartonellosis. Carrión's disease is geologically limited, affecting high altitude Andean communities of Peru, Ecuador, and Colombia and is characterised by two distinct clinical syndromes. After an average incubation period of 60 days, Oroya fever, the acute phase, occurs, characterised by fever, haemolytic anaemia, pallor, myalgia, and $\operatorname{arthralgia}^{14}$. B. bacilliformis is the only known Bartonella species to cause red blood cell lysis in the acute phase of infection, a dangerous symptom resulting in an often-fatal haemolytic anaemia. The chronic phase, known as Peruvian wart, verruga andícola, or verruga peruana, occurs weeks to years following infection. Verruga peruana is termed the eruptive phase and can occur without any history of Oroya fever. Large numbers of pleomorphic nodular lesions appear over the body, particularly the arms and legs, accompanied by systemic symptoms: fever, malaise, osteoarticular pain, and headaches ${ }^{14}$. Verruga peruana has been observed in ancient mummies of human sacrificial rituals in Peru ${ }^{15}$. Diagnosis of Carrión's disease requires 
microscopy of infected erythrocytes; once identified the patient is promptly treated with the antibiotic chloramphenicol ${ }^{14}$. B. bacilliformis shares several similarities with other Bartonella spp., such as arthropod vector transmission, red blood cell parasitism, skin lesions, and the characteristic acute and chronic phases of illness. However, as case fatality rates of Carrión's disease infection can exceed $80 \%$, predominantly affecting children, B. bacilliformis infection is far more severe than infections with other Bartonella species, and is recognised as a neglected tropical disease ${ }^{13}$.

\subsubsection{B. henselae}

B. henselae is the most common Bartonella spp. to infect humans ${ }^{12}$. Cats are the reservoir host of $B$. henselae, and transmission between cats occurs by cat flea (Ctenocephalides felis) ${ }^{16-18}$. The majority of $B$. henselae infections of cats are sub-clinical; however, B. henselae infection in cats can become chronic, persisting in the bloodstream for months ${ }^{19,20}$, or even exceeding one year ${ }^{21}$. Atypical symptoms such as endocarditis have been reported but not investigated thoroughly ${ }^{19,22}$. B. henselae is transmitted to incidental hosts such as humans or dogs through cat scratches or bites, resulting in cat scratch disease (CSD). The condition is more common in children between the ages of five and nine ${ }^{23}$, likely because they receive more cat scratches. Although most CSD cases are mild and self-limiting, a subset of cases can become chronic and far more serious, occurring more frequently in immunocompromised patients and children under five years ${ }^{24}$. Serious complications of $B$. henselae infection include bacillary angiomatosis, characterised by the presence of pseudo-neoplastic and vasoproliferative lesions ${ }^{5}$ and peliosis hepatis, where blood-filled cavities develop throughout the liver. B. henselae has also been associated with chronic asymptomatic bacteraemia in humans, characterised by isolation of bacteria from the blood. However, these diseases are almost exclusively associated with an immunocompromised state, such as HIV-infected patients or post-transplant patients receiving immunos uppressive treatment ${ }^{25}$.

\subsubsection{B. quintana}

B. quintana, which causes trench fever, infects humans as the reservoir host and is spread by the body louse (Pediculus humanus humanus). In addition, B. quintana causes opportunistic infections in immunocompromised patients, causing serious disease such as bacillary angiomatosis. Risk factors for B. quintana infection include alcoholism, cold exposure, a suppressed immune system, and poor hygiene. As a result, B. quintana infection is becoming 
Chapter 1 Introduction

common in homeless and socially disadvantaged populations under the name urban trench fever.

\section{History}

B. quintana was first recorded in 1915 as a widespread pathogen causing serious illness to the troops of World War I, responsible for the disease termed trench fever ${ }^{26,27}$. It was estimated the average weekly evacuation of troops from France due to trench fever was $0.6 \%$ of British army strength $^{26}$. During World War I more than 1 million soldiers were rendered unfit for duty for greater than 60 days by trench fever ${ }^{27}$. The causative agent of trench fever had yet to be identified. The disease shared similarities with malaria; it was proposed the causative agent may also be transmitted by a fly or parasite found in the trenches ${ }^{28}$. In 1918 the body louse was confirmed as the vector for trench fever when collaborative British and American physicians demonstrated the ability of the louse to transmit the disease through excreta ${ }^{26}$. B. quintana has seen numerous name changes since its discovery; during World War I significant resources were assigned to research the causative agent of trench fever, then known as Rickettsia. It was quickly recognised the best way to combat trench fever was to eliminate louse exposure. The burden of trench fever on the war effort was reduced during final years through the use of antilouse measures such as the sterilisation of uniforms using steam ${ }^{26}$. In addition, regular washing of uniforms and bedding with hot water was crucial in reducing incidence of trench fever. Trench fever returned during World War II, particularly in German troops ${ }^{29}$, but the use of lousicides such as DDT by the soldiers reduced the impact of the disease ${ }^{27}$. Additionally, World War II involved less prolonged crowding of soldiers, resulting in reduced louse contact between soldiers compared to the conditions of World War $\mathrm{I}^{27}$. In 1961 Rickettsia quintana was successfully cultured in a laboratory setting and moved to the genus Rochalimaea ${ }^{27}$. With the development of $16 \mathrm{~S}$ phylogenetic analysis Rochalimaea quintana was discovered to be a relation of $B$. bacilliformis ${ }^{30}$ and was once again reclassified under the genus Bartonella ${ }^{29}$. Although B. quintana was identified as a formidable human pathogen during World War I, the bacterium has infected humans for much longer. B. quintana infected soldiers of Napoleon's Grand Army of the early 1800's as they retreated from Russia ${ }^{31}$. Furthermore, B. quintana DNA was isolated from dental pulp retrieved from mass graves resulting from the early 1700's, War of Spanish Succession ${ }^{32}$ and 4000-year-old dental pulp from France ${ }^{33}$. This discovery reveals B. quintana is one of humanity's most ancient bacterial pathogens, along with tuberculosis ${ }^{33}$. In the absence of $B$. quintana affecting large scale troops, medical interest dropped dramatically ${ }^{29}$. Trench fever was thought to be an illness of the past; however, rates of $B$. quintana infection have surged in recent years in socially disadvantaged populations, renewing 
the infection under a new name, urban trench fever ${ }^{34}$. Epidemics have erupted in homeless populations and refugee camps. With this re-emergence, research onto B. quintana has once again become imperative to medicine.

\section{Transmission and Risk Factors}

It was established in 1918 that trench fever was transmitted by the human body louse (Pediculus humanus humanus). Although B. quintana DNA has been detected in the head louse (Pediculus humanus capitis), this vector is not known to be competent for transmission ${ }^{35,36}$. Ticks have not been shown to transmit B. quintana to humans ${ }^{37}$. The bacteria are not transmitted directly from the louse, but are prevalent in the louse excreta, deposited at the site of feeding. The bacteria then enter the body through broken skin, aided by scratching or rubbing ${ }^{38}$. The bacteria colonise the bloodstream and invade erythrocytes in a host-specific manner, then persist intracellularly ${ }^{12}$. Infected red blood cells are taken up by blood-feeding arthropods, such as the human body louse. The bacteria are released in the gut of the arthropod, replicate, and infect hosts during subsequent blood meals. The risk factors for B. quintana infection include homelessness, poor hygiene, alcoholism, prolonged cold temperatures, compromised immune system, and body louse exposure. While homelessness and poor hygiene are risk factors for body louse exposure, cold temperatures and a compromised immune system are risk factors for more severe and persistent infection, once exposed. As the homeless population have the majority of these risk factors, they are particularly susceptible to louse infestation and B. quintana infection ${ }^{39}$. Other socially disadvantaged populations such as those residing in refugee camps or living in developing areas are at risk resulting in $B$. quintana epidemics ${ }^{34}$. Once infected with $B$. quintana, underlying health factors common among the homeless likely increase their risk to develop the more serious diseases associated with infection ${ }^{34}$. A 2014 study showed the prevalence of lice within the homeless population was extremely high; $30 \%$ of tested individuals were infested with lice, while $16 \%$ of these lice tested positive for B. quintana ${ }^{39}$.

\section{Infection}

Infection of an immunocompetent person by $B$. quintana typically results in trench fever. Trench fever, also known as quintana fever, 5-day fever, or Volhynia fever, is characterised by bacteraemia accompanied by fevers lasting one to three days, and recurring every four to six days, usually reducing in severity with each subsequent fever ${ }^{34,40}$. The incubation period of trench fever varies between 15 and 25 days $^{29}$. Serious disease phenotypes and complications are far more likely if the patient is immunocompromised. Severe clinical manifestations of $B$. quintana infection occur in immunocompetent patients but occur more frequently when the 
patient is immunocompromised ${ }^{12,16}$. These include: chronic bacteraemia, infective endocarditis, bacillary angiomatosis, and lymphadenopathy ${ }^{4}$. Chronic bacteremia is when B. quintana is present in the blood for extended durations; in the case of $B$. quintana this has been observed for up to 8 years ${ }^{29,41,42}$. Recent studies suggest the duration of B. quintana associated bacteraemia is likely up to 78 weeks ${ }^{43}$. Intermittent bacteraemia has also been observed, with the bacteria returning after periods of apparent clearance. Infective endocarditis is characterised as the colonisation of a prosthetic or native heart valve by microorganisms, most commonly bacteria $^{44}$. One-year mortality rate following diagnosis of endocarditis remains at $30 \%$, which is greater than many cancers ${ }^{44}$. Diagnosis of infective endocarditis is challenging, both blood cultures and oesophageal echocardiography are implemented to identify the causative agent ${ }^{45}$. When the causative agent cannot be identified by these methods it is referred to as culture negative endocarditis; this occurs in $2.5-31 \%$ of cases ${ }^{45}$. B. quintana is one of the most common pathogens responsible for culture-negative endocarditis ${ }^{46-50}$. There are two main reasons the pathogen causing endocarditis is not identified by culture: first, the patient may be receiving antibiotic treatment for systemic bacterial infection. Second, the causative microorganisms have limited proliferation in conventional culture media ${ }^{45}$. In a study of 819 patients diagnosed with culture negative endocarditis between the years of 2001 - 2009 serology and PCR diagnostic approaches were implemented to identify the microorganisms responsible in each case. Bartonella spp. were identified in $19.2 \%$ of cases $^{51}$, although prevalence of $B$. quintana endocarditis varies around the world due to varying endemicity of other agents of culture-negative endocarditis. The interactions between Bartonella spp. and the human host, resulting in infective endocarditis, are largely unexplored. Bacillary angiomatosis is a proliferative vascular disease affecting the liver, spleen, lymph nodes, bone marrow, and most commonly the $\operatorname{skin}^{41}$. This is a common phenotype in extremely immunodeficient patients infected with B. quintana, such as those co-infected with the HIV virus ${ }^{29}$. B. quintana associated bacillary angiomatosis is associated with bone lesion and subcutaneous masses. This disease is

life threatening if left untreated. In rare cases B. quintana causes lymphadenopathy, an expansion of lymph nodes, due to a granulomatous reaction to the bacteria.

\section{Diagnosis and Treatment}

B. quintana infection should be considered as a diagnosis if the patient has risk factors, such as urban homelessness, and if the patient develops non-localising illness of an unknown cause ${ }^{34}$. Infection can be presumed through symptomology and contact history with body louse; to confirm, laboratory identification of $B$. quintana in the patient tissue, blood, or serum samples is required. Diagnosis requires specific culture, nucleic acid amplification, or serological 
methods ${ }^{34}$. Diagnosis through culture is challenging as the bacteria are fastidious and slowgrowing, thus diagnosis takes approximately 21 days. Serology is frequently used in diagnosis; however, these tests often have low sensitivity and specificity, resulting in high rates of false positives and negatives. Serological cross-reactions can occur between $B$. henselae, $B$. quintana, and Coxiella burnetii species, which is problematic as treatments differ between these infections $^{52}$. PCR amplification of $B$. quintana specific targets is another implemented diagnostic tool. These three techniques can be used in combination for a more confident diagnosis $^{53}$. To date, no single treatment strategy is shown to be effective at treating all Bartonella related clinical syndromes ${ }^{54}$, although various combination antibiotic treatments have been shown effective with adherence. Bacteraemia caused by B. quintana is treated with a combination of gentamicin and doxycycline ${ }^{55}$. Vasopoliferative lesions are treated with erythromycin; however, lymphadenopathy does not significantly improve with antibiotic treatment ${ }^{4}$.

\subsection{Life Cycle}

The infection cycle for Bartonella begins when the mammalian host is bitten by an infected blood-feeding arthropod, such as a flea or louse. Once the bacteria enter the dermis of the skin they encounter immune cells that are fixed within the tissues. It should be noted that most studies have been conducted with zoonotic Bartonella species, usually in their reservoir host; it's not fully known if the life cycle of B. quintana infection in humans occurs the same way. Some Bartonella are phagocytosed by sentinel innate immune cells such as dendritic cells or macrophages; the degree to which the bacteria can persist within these innate immune cells is largely unknown. Kyme et al. demonstrated B. henselae-containing vacuoles within murine macrophages and human dendritic cells can prevent lysosome fusion ${ }^{56}$, improving intracellular survival. Dissemination of $B$. henselae, allowing the bacteria to establish infection, is dendritic cell-dependent ${ }^{57}$. Dendritic cells may intracellularly transport bacteria from the site of infection. Bartonella enter draining lymph nodes, allowing the bacteria to gain access to the bloodstream ${ }^{58-60}$. Typically, five days post infection, large numbers of bacteria enter the bloodstream and continue to be released in synchronous waves at intervals of approximately five days ${ }^{58,60,61}$. Cessation of this process results from a specific antibody response, shown in the mouse model infected with $B$. grahamii $^{62}$. During infection of a reservoir host, Bartonella invades and persists within erythrocytes ${ }^{20,58}$, resulting in persistent bacteraemia ${ }^{8,63}$. Bartonella are unable to invade erythrocytes during infection of incidental hosts ${ }^{58}$. Bartonella multiply intracellularly within erythrocytes until the number of bacteria reaches a plateau; the number of bacteria per erythrocyte varies among Bartonella species ${ }^{58}$. Erythrocyte invasion by 
Bartonella does not appear to reduce the lifespan or impair the function of the erythrocyte, which continue to circulate ${ }^{58}$. The major exception to this is B. bacilliformis, whichinvades and eventually lyses erythrocytes, causing a fatal haemolytic anaemia. Bartonella can persist for months, or even years in the bloodstream, allowing transmission to further biting arthropods. Bartonella spp. survive using strategies to evade, and possibly actively suppress, the host immune system. The replicative niche, also known as the blood-seeding niche, refers to the cell types and locations in the body where Bartonella spp. replicate and persist, both post inoculation and during chronic infection. This niche has been proposed to be vascular endothelial cells, which the bacteria interact with, and which are involved in several Bartonellarelated pathologies, such as bacillary angiomatosis lesions. Bartonella infections are often subject to periods of apparent clearance, followed by relapse, indicating that Bartonella are protected against immune system assault within the replicative niche, then re-seed the bloodstream $^{12}$. This protection has been suggested to result from intracellular persistence, although bacteria have not been observed inside non-erythrocytic cells in experimental or natural infections. A careful study using an immunocompromised mouse model sugges ted that the bacteria persist in the extracellular space, closely associated with vascular endothelial cells and the extracellular matrix ${ }^{64}$. As the majority of Bartonella spp. lack flagella, invasion of migratory cells such as erythrocytes or migratory immune cells, is beneficial. This allows passive transport of bacteria to sites where Bartonella can replicate and persist; a likely site is microvasculature ${ }^{12}$. When an uninfected body louse takes a blood meal from an infected host Bartonella will be ingested and replicate within the body louse gut, continuing the life cycle.

\subsection{Immunology \& Immune Evasion by Bartonella}

The human immune system is a complex network of specialised cells, both adaptive and innate, that protect the body from foreign invaders. The innate immune system comprises a range of cells that rapidly destroy foreign micro-organisms. The clear majority of fixed sentinel cells in the tissues are members of the innate immune system, including macrophages and specialised dendritic cells called Langerhans cells. When the skin is broken, and bacteria enter the dermis, they encounter these phagocytes. Phagocytes respond to bacterial antigens through toll-like receptors (TLRs) bound to the plasma membrane, which recognise pathogen-associated molecular patterns (PAMPs) associated with bacteria. Typically, PAMP stimulation activates the Mitogen-activated Protein Kinase (MAPK) signalling cascade promoting pro-inflammatory signalling, such as cytokine secretion. Macrophages are present in the dermis of the skin, acting as sentinels ready to engulf and destroy invaders as they enter any breach in the outer skin layer. Bartonella typically enter the body though a scratch on the skin where they encounter 
macrophages. Although macrophages are adept at recognising and killing bacteria through phagocytosis, Bartonella spp. survive these interactions and spread to the bloodstream, through mechanisms that remain largely unexplored. One known evasion mechanism of Bartonella is its unique lipopolysaccharide (LPS). Experimental challenge of human monocytes with $B$. quintana demonstrated that its LPS is an antagonist to TLR-4, drastically inhibiting mRNA expression of pro-inflammatory molecules, including TNF- $\alpha$, IL-1 $\beta$, and IL- $6{ }^{65}$. The extent of this inhibitory effect indicated B. quintana LPS may prevent uptake by macrophages, allowing the bacteria to evade immune cell killing. In addition, reduced pro-inflammatory cytokine secretion by innate immune cells would impede the immune response against Bartonella. This suppression of TLR-4 signalling is also likely essential for Bartonella persistence in the bloodstream, as other gram-negative organisms would cause an overwhelming immune response and septic shock ${ }^{25}$. The interactions between phagocytes and Bartonella may be very species-specific, particularly between reservoir and incidental host infection. Therefore, the

interactions between $B$. quintana and human macrophages should be characterised to confidently understand the early stages of infection in the reservoir host. Macrophage activity is altered by Bartonella spp. during infection ${ }^{66,67}$, although the interactions between B. quintana and human macrophages remain uncharacterised.

\subsection{Bartonella Virulence Factors}

Virulence factors are molecules produced by pathogens that contribute to the pathogenicity of the organism. Bartonella spp. are equipped with a wide range of virulence factors, improving their ability to colonise, replicate, and persist within a host ${ }^{68}$. These include secretion systems, effector proteins, trimeric autotransporter adhesins, and flagella. There are three different type IV secretion systems (T4SSs) found in Group C Bartonella spp: VirB/D4, Trw, and Vbh ${ }^{12,69}$. These secretion systems are expressed in place of the flagella present in the other Bartonella lineages. B. henselae and B. quintana possess both Trw T4SS and VirB/D4 T4SS. The Trw T4SS forms a pilus-like structure on the cell surface and plays a role in the colonisation of erythrocytes $^{12}$. The Trw T4SS is responsible for the host-restricted adhesion of the bacteria to erythrocytes of the reservoir host ${ }^{70}$. Trw T4SS is confined to Bartonella spp. from Group C and B. australis and is mutually exclusive with the flagella ${ }^{11,12}$. A known target of Trw T4SS is band3, a major outer membrane protein of erythrocytes ${ }^{71}$. The well characterised VirB/D4 T4SS is the most common found in Bartonella spp., is expressed in Group B and C, and secretes Bartonella effector proteins (Beps) directly into host cells ${ }^{12}$. There have been seven Beps identified in Bartonella spp. named BepA - G, which modify host cells signalling and are essential for infection ${ }^{12}$. B. henselae encodes all seven whereas $B$. quintana encodes five. $B$. 
quintana additionally encodes a homologue of the Yersinia YopJ secreted effector protein ${ }^{72}$, known to downregulate the host cell NF- $\mathrm{KB}$ and MAPK pathways, disrupting innate immune function $^{73}$; however, the role of the B. quintana YopJ homologue protein is still unclear. Deformin, also known as deformation factor, is a virulence factor secreted by B. bacilliformis that deforms the erythrocyte membrane creating invaginations and trenches which internalise bacteria $^{74,75}$. A deformin homologue with reduced activity is expressed in B. henselae ${ }^{74}$. Trimeric autotransporter adhesins mediate adhesion to host cells, an important step during bacterial infection. In B. henselae this adhesion is provided by Bartonella adhesin A (BadA). BadA mediates autoagglutination, host cell adhesion, binding of components of the extracellular matrix, and induction of a proangiogenic response by host cells ${ }^{76} . B$. henselae also encodes filamentous hemagglutinins (fhaB1-8), proposed to influence adhesion ${ }^{76,77}$. $B$. quintana encodes a family of variably expressed outer-membrane proteins (Vomps), analogous to the BadA protein of $B$. henselae, which mediate adhesion and autoaggregation ${ }^{78}$. Vomps adhere to extracellular matrix components such as fibronectin and collagen ${ }^{79}$. Interestingly, $B$. quintana JK31 increases vascular endothelial growth factor secretion from THP-1 and HeLa 229 cells in a Vomp-dependent manner; however, it does not exhibit increased cell adhesion, compared to B. quintana Vomp-negative strains $^{80}$. B. quintana JK31 Vomps do not share the exact same function as the homologue $B$. henselae BadA, but instead play a role in angiogenic reprogramming ${ }^{80}$. Vomps are closely related to the afimbrial adhesin YadA of Yersinia enterocolitica, and have been shown to be essential for bloodstream infection in a rhesus macaque animal model ${ }^{78}$. Flagella are present in Bartonella spp. from Group A and $\mathrm{B}^{11}$. The flagella enable greater motility and aid invasion; for example, the flagella of B. bacilliformis contribute to the invasion of erythrocytes by the bacteria ${ }^{81}$.

\subsection{Role of Bartonella Type IV secretion system in Virulence}

T4SS are macromolecular protein complexes that inject protein or nucleic acid complexes directly into host cells. T4SS are present in a range of gram-negative bacteria and evolved from conjugation machinery ${ }^{12}$. The secretion apparatus is membrane bound and forms a translocation channel, spanning the inner and outer bacterial membranes and the eukaryotic host cell membrane ${ }^{82}$. The T4SS can transport effector proteins or DNA from the cytoplasm of the bacteria directly into the host cell cytoplasm ${ }^{12,83}$. The VirB/D4 T4SS in B. quintana is encoded by the virB locus, comprised of 10 structural genes and a virD4 gene. In Group C Bartonella spp., the virB locus is located upstream of the secreted Bep genes, encoding proteins that form the secretion complex. The virD4 gene is located amidst the Bep genes, encoding the VirD4 protein, which associates with the inner membrane side of the T4SS complex. VirD4 recognises 
the Bartonella intracellular delivery (BID) domains of the Bep proteins and allows their entry into the channel to be transported ${ }^{84}$. The BID domain is a 142-amino acid sequence that, in combination with the positively charged C-tail, acts as a translocation signal, among other functions, allowing the protein to be transported through the VirB/D4 T4SS ${ }^{85,86}$. Each Bep contains at least one BID domain; in B. henselae three Beps have more than one BID domain; however, the BID domain closest to the C-terminus enables the translocation ${ }^{86}$. This suggests the other BID domains within the Beps have other activities. Acquisition and evolution of the different Bartonella T4SS effectors have likely occurred through repeated instances of horizontal gene transfer, often promoted by gene transfer agents ${ }^{11}$. The VirB/D4 T4SS is essential for intraerythrocytic infection in animal models. It also mediates multiple phenotypes in endothelial cells, including activation of $\mathrm{NF}-\kappa \mathrm{B}$ to promote a pro-inflammatory response, cytoskeletal rearrangements, apoptosis inhibition, and pathogenic angiogenesis ${ }^{87-89}$. Bartonella spp. of Group C have a pathogenicity island encoding the highly conserved VirB/D4 T4SS and the Bartonella effector proteins (Beps) ${ }^{86}$. Interactions between Bartonella spp. and the reservoir host create a strong selection pressure promoting rapid evolution of the Beps. The Beps are not highly conserved between Group C species and differ in the number encoded. The Beps share a common ancestor and display a modular domain architecture ${ }^{23}$, and likely arose from multiple rounds of duplication, reshuffling, and diversification of domains ${ }^{12}$. Bartonella likely acquired the T4SS and early Bep proteins from several independent events ${ }^{90}$. Bep proteins have an $\mathrm{N}$ terminal host interacting domain and a C-terminal bipartite secretion signal containing a BID domain $^{12}$. The system is essential for $B$. henselae infection; BepG mediates actin cytoskeleton rearrangements driving the formation of a large vacuole, densely packed with bacteria called the invasome and is essential for intraerythrocytic infection ${ }^{91}$. BepE and BepC work in concert; $\mathrm{BepC}$ is required for Bartonella dissemination while BepE protects migratory cells from the harmful effects caused by BepC ${ }^{57}$.

\subsubsection{BepA Homologue Effectors}

B. henselae BepA is well studied and contains both a BID, allowing passage through the VirB/D4 T4SS, and a filamentation induced by cAMP (FIC) domain, an enzymatic domain associated with pathogenic manipulation of host cell signalling ${ }^{92}$. When BepA is secreted into host cells it localises to the inner membrane, binding adenyl cyclase and the $\alpha$-subunit of a Gprotein coupled adenyl cyclase stimulating receptor, elevating intracellular cAMP ${ }^{93}$. BepA binds adenyl cyclase via its BID domain to perpetuate cAMP production, inhibiting apoptosis by the upregulation of cAMP-dependent anti-apoptotic signalling proteins ${ }^{93}$. BepA demonstrates a unique manner of cAMP production in host cells, in contrast to other microbial 
mechanisms such as ADP ribosylation by cholera toxin ${ }^{93}$. Additionally, B. henselae BepA has been shown to have a potent pro-angiogenic effect, contributing to $B$. henselae's chronic persistence within the host and may promote the development of vasopoliferative lesions during serious $B$. henselae infection ${ }^{89}$. The bepA gene homologue in $B$. quintana differs from that of $B$. henselae as it is expressed as two open reading frames, resulting in two proteins, BepA1 and BepA2. The first open reading frame, BepA1, encodes the FIC domain and the second contains the BID domain with the C-terminus tail ${ }^{88}$. The functionality or potential cellular targets of BepA1 have not been demonstrated.

FIC enzymatic domains are commonly found in cells, occurring in thousands of different species, often modulating signalling processes. FIC domains mediate the transfer of an AMP moiety onto a target protein. They were first characterised in the early 1970's when Stadtman et al. identified an E. coli glutamine synthetase that was regulated by an AMPylating enzyme ${ }^{94,95}$. More recently the role of FIC domains in bacterial pathogenesis started to come to light. In 2009 Yarbrough et al. demonstrated the ability of bacterial FIC domains to alter the function of host cell Rho signalling family members. AMPylation, also known as adenylylation, is the covalent transfer of an AMP moiety to a target protein side chain, a modification which has recently become more recognised to influence bacterial pathogenicity and toxicity ${ }^{96}$. Physiologically, AMPylation mediates posttranslational regulation of protein function; however, microbial pathogens have subverted this activity to modulate host cellular function during infection ${ }^{92}$. Bacterial toxins containing FIC domains have been demonstrated to interfere with Rho guanine triphosphatase (GTPase) family function and disrupt microtubule-dependent vesicle transport ${ }^{92}$. FIC domains are also often part of toxin/anti-toxin modules in plasmids; for example, the FIC domain of B. schoenbuchensis VbhT has a toxic effect but is intermolecularly suppressed by the function of VbhA via tight binding of its $\alpha_{\text {inh }} s i^{97}$. Additionally, the FIC domain-containing effector protein Bep2 of B. rochalimae targets the intermediate filament protein Vimentin ${ }^{98}$.

\subsection{Hypothesis \& Goals}

Once Bartonella are introduced into the skin they encounter resident macrophages, but survive clearance using poorly understood immune evasion or suppression mechanisms. An intriguing question is whether B. quintana are phagocytosed by resident macrophages and survive within them, or whether the bacteria evade the immune cells altogether. B. henselae have demonstrated the ability to persist intracellularly within immune cells, preventing fusion of the lysosome; perhaps $B$. quintana persists within macrophages utilising a similar mechanism. B. quintana is 
Chapter 1 Introduction

equipped with five Bep proteins that may modulate host cell signalling to benefit the bacteria. Perhaps these Bep proteins play a role in the modulation of host immune cells, allowing evasion or suppression of immune activity. The interactions between $B$. quintana and human phagocytes have not been characterised. We hypothesised that B. quintana would be internalised by human macrophage cells but persist intracellularly. We further hypothesised that BepA1 and BepA2 would modulate host cell protein functions, affecting cellular signalling. Our goals were to investigate the interactions between $B$. quintana and human macrophages through live cell assays and to identify protein-protein interactions between BepA1/2 and host cell proteins to characterise their cellular target(s). Expanding on our findings, we also assessed the effects of BepA1/2 on host cells using functional assays. 


\section{Chapter 2 Methods}

\subsection{Bacterial and Yeast Strains and Plasmids}

B. henselae and B. quintana were cultured for use in cell infection experiments. E. coli were used as host organisms for the generation and maintenance of plasmids.

\begin{tabular}{|c|c|c|}
\hline \multicolumn{3}{|c|}{ E. coli Strains } \\
\hline Strain & Genotype & Source or Reference \\
\hline DH5 $\alpha$ & $\begin{array}{l}\mathrm{F}-\Phi 80 \text { lacZ } \Delta \mathrm{M} 15 \Delta(\text { lacZYA-argF) } \\
\mathrm{U} 169 \text { rec } 11 \text { endA1 hsd } \mathrm{R} 17 \text { (rK-, } \\
\mathrm{mK}+) \text { phoA supE44 } \lambda-\text { thi } \\
1 \text { gyrA96 relA1 }\end{array}$ & ThermoFisher Scientific \\
\hline Top10 & $\begin{array}{l}\mathrm{F}-\text { mcrA } \Delta(\text { mrr-hsdRMS-mcrBC }) \\
\text { Ф80lacZ } \Delta \mathrm{M} 15 \\
\Delta l a c \mathrm{X} 74 \text { recA1 araD139 } \Delta(\text { ara leu }) \\
7697 \text { gal } \mathrm{U} \text { galK } r p s \mathrm{~L} \\
\text { (StrR) endA1 nupG }\end{array}$ & ThermoFisher Scientific \\
\hline
\end{tabular}

\begin{tabular}{|l|l|l|}
\hline \multicolumn{2}{|l|}{ Bartonella Strains } \\
\hline Strain & Relevant characteristics & Source or Reference \\
\hline B. henselae & $\begin{array}{l}\text { Isolated from human blood from an } \\
\text { Houston-1 }\end{array}$ & 99 \\
& HIV-positive male, Houston, Texas, \\
USA. & $\begin{array}{l}\text { Source - ESR Culture } \\
\text { Collection. }\end{array}$ \\
\hline B. quintana JK-31 & $\begin{array}{l}\text { Isolated from a cutaneous bacillary } \\
\text { angiomatosis lesion of a human } \\
\text { patient in San Francisco, California, } \\
\text { USA. }\end{array}$ & $\begin{array}{l}\text { BEI Resources, NIAID, NIH } \\
\text { (NR-31832). }\end{array}$ \\
\end{tabular}




\begin{tabular}{|c|c|c|}
\hline \multicolumn{3}{|c|}{ Yeast Strains } \\
\hline Strain & Relevant characteristics & Source or Reference \\
\hline Y187 & 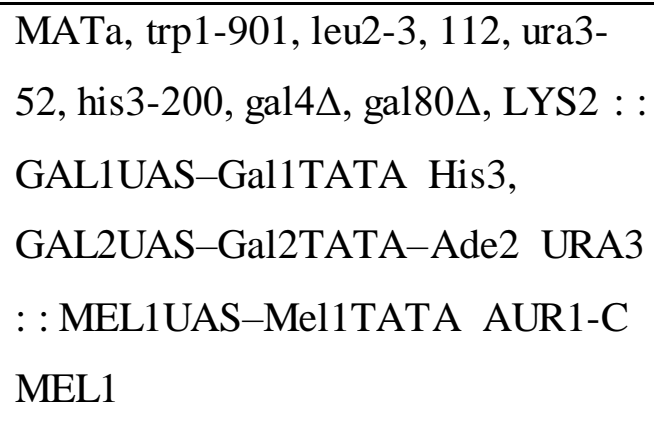 & Takara Bio USA \\
\hline Y2HGold & $\begin{array}{l}\text { MAT } \alpha \text {, ura3-52, his3-200, ade2-101, } \\
\text { trp1-901, leu2-3, 112, gal4 } \Delta \text {, gal80 } \\
\text { met-, URA3 : : GAL1UAS- } \\
\text { Gal1TATA-LacZ, MEL1 }\end{array}$ & Takara Bio USA \\
\hline
\end{tabular}

\begin{tabular}{|c|c|c|}
\hline \multicolumn{3}{|l|}{ Plasmids } \\
\hline Name & Relevant characteristics & Source or Reference \\
\hline pGADT7-T & Y2HS control plasmid & Takara Bio USA \\
\hline pGBKT7-53 & Y2HS control plasmid & Takara Bio USA \\
\hline pGBT9 & $\begin{array}{l}\text { Y2HS low copy number cloning } \\
\text { plasmid }\end{array}$ & Takara Bio USA \\
\hline pGBKT7 & Y2HS cloning plasmid & Takara Bio USA \\
\hline pGADT7-RecAB & Y2HS library plasmid & Takara Bio USA \\
\hline pGBKT7-BepA1 & pGBKT7 with BepA1 & This study \\
\hline pCR®2.1-TOPO® & Topo cloning plasmid & ThermoFisher Scientific \\
\hline
\end{tabular}

\begin{tabular}{|c|c|c|}
\hline \multicolumn{3}{|c|}{ Mammalian $E$. coli Shuttle Vectors } \\
\hline Name & Relevant characteristics & Source or Reference \\
\hline pcDNA3.1 & $\begin{array}{l}\text { Mammalian- } E \text {. coli shuttle vector for } \\
\text { transfection. }\end{array}$ & ThermoFisher Scientific \\
\hline pcDNA3.1-BepA1 & pcDNA3.1 with BepA1 & This study \\
\hline $\begin{array}{l}\text { pcDNA3.1-GFP- } \\
\text { yopJ-catmut }\end{array}$ & $\begin{array}{l}\text { Unpublished lab stock plasmid; } \\
\text { pcDNA }^{\mathrm{TM}} 3.1 \mathrm{CT}-\mathrm{GFP}-\mathrm{TOPO}{ }^{\circledR} \text { vector } \\
\text { containing catalytic mutant YopJ. }\end{array}$ & $\begin{array}{l}\text { pcDNA }{ }^{\mathrm{TM}} 3.1 / \mathrm{CT}-\mathrm{GFP}- \\
\text { TOPO® sourced from } \\
\text { ThermoFisher Scientific. } \\
\text { Catalytic mutant YopJ } \\
\text { added by Alvey Little. }\end{array}$ \\
\hline
\end{tabular}


Chapter 2 Methods

Table 1: Strains and Plasmids. This table presents the different bacterial and yeast stains used during this research. Additionally, plasmids used and created are presented with their relevant characteristics noted.

\section{Bacteriological Media}

All bacteriological media was autoclaved for 15 minutes at $121^{\circ} \mathrm{C}, 15$ psi.

Chocolate Agar

The GC agar and haemoglobin solutions were brought to the boil separately then autoclaved. Solutions were cooled to $45^{\circ} \mathrm{C}-50^{\circ} \mathrm{C}$, then combined in a biosafety cabinet.

$500 \mathrm{~mL}$ of $7.2 \%$ GC Agar Base Solution (OXOID)

$500 \mathrm{~mL}$ of $2 \%$ BBL $^{\mathrm{TM}}$ Freeze-dried Haemoglobin Solution (BD)

1\% IsoVitaleX Enrichment Medium (BD) added once autoclaved solutions had cooled. Plates were poured in a biosafety cabinet; $29 \mathrm{~mL}$ of liquid chocolate agar was pipetted into each empty petri dish. Lids were left ajar for 15 minutes allowing condensation to escape while plates cooled and solidified. Plates were stored inverted at $4^{\circ} \mathrm{C}$ for up to 7 days for use in B. quintana culture.

\section{Columbia Blood Agar (CBA)}

Sleeves of pre-made CBA plates with 5\% sheep blood were purchased from Fort Richard Laboratories.

\section{$\underline{\text { LB Agar }}$}

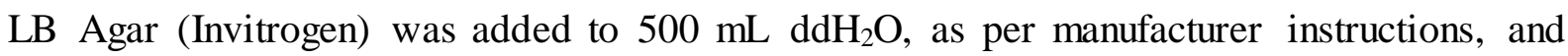
autoclaved. The solution was cooled to $50^{\circ} \mathrm{C}$ then antibiotics were added, if required: Kanamycin $50 \mu \mathrm{g} / \mathrm{mL}$, Ampicillin $50 \mu \mathrm{g} / \mathrm{mL}$ (final concentrations). In a biosafety cabinet, 21 $\mathrm{mL}$ agar was pipetted into each $90-\mathrm{mm}$ sterile petri dish (Citotest). The lids were left ajar until agar had solidified then plates were stored at $4{ }^{\circ} \mathrm{C}$ for up to one month.

\section{$\underline{\text { LB Broth }}$}

LB broth powder was added to $500 \mathrm{~mL} \mathrm{ddH}_{2} \mathrm{O}$, as per manufacturer instructions, and autoclaved. The solution was cooled to $50^{\circ} \mathrm{C}$ then antibiotics were then added if required: Kanamycin $50 \mu \mathrm{g} / \mathrm{mL}$, Ampicillin $50 \mu \mathrm{g} / \mathrm{mL}$.

$\underline{2 x Y T}$

Difco $^{\mathrm{TM}}$ 2xYT Yeast Extract Tryptone Medium (BD) was added to $500 \mathrm{~mL} \mathrm{ddH}_{2} \mathrm{O}$, as per manufacturer instructions, and autoclaved.

\section{$\underline{\mathrm{M} 199 \mathrm{~S}}$}

Glutamate (final concentration $1 \mu \mathrm{M}$ ) (Gibco, ThermoFisher), sodium pyruvate (Gibco, ThermoFisher) $1 \mathrm{~mL}$ and FBS (final concentration 20\%) were added to M199 Earle's Salt 
Chapter 2 Methods

Medium (Gibco, ThermoFisher), to a final volume of $100 \mathrm{~mL}$. The solution was then filter sterilised.

$\underline{\text { TSB Broth }}$

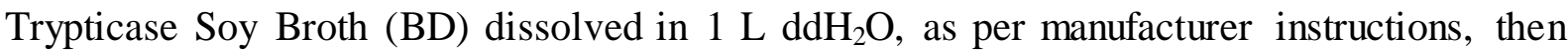
autoclaved.

\section{Bartonella}

All maintenance of Bartonella was performed within a AES Environmental - Biological Safety Cabinet Class II Hood. B. henselae stocks were kept in $-80^{\circ} \mathrm{C}$ storage until required. To revive bacteria, a scraping of frozen stock was taken using a sterile inoculation loop and spread over a CBA plate, then incubated. Stock plates were passaged every $3-4$ days by scraping $\sim 1 / 3$ of the plate using a sterile inoculation loop and spreading over a fresh CBA plate, then incubating. Strains were sub-cultured up to 10 times before returning to frozen stocks. B. henselae was grown in a humidified incubator at $37^{\circ} \mathrm{C}$ inside a glass jar with a lit candle to reduce $\mathrm{O}_{2}$ and increase $\mathrm{CO}_{2}$. To freeze $B$. henselae cultures, approximately $1 / 3$ of a 3 - 4-day old plate was collected using a sterile inoculation loop and placed into $1 \mathrm{~mL}$ bacterial freezing media (80\% TSB broth, $20 \% 80 \%$ glycerol), vortexed briefly, then placed into $-80^{\circ} \mathrm{C}$ storage. B. quintana stocks were revived and maintained in the same manner as B. henselae, although B. quintana cultures were grown on chocolate agar media and frozen in 80\% M199S with 20\% DMSO.

\section{E. coli}

To culture, a single colony was resuspended in $5 \mathrm{~mL} \mathrm{LB}$ broth and grown overnight at $37^{\circ} \mathrm{C}$ with shaking at $200 \mathrm{RPM}$. For long term storage, $600 \mu \mathrm{L}$ overnight culture was added to 400 $\mu \mathrm{L} 80 \%$ Glycerol in a $1.5 \mathrm{~mm}$ cryo vial (Tarsons), then frozen at $-80^{\circ} \mathrm{C}$. Plasmids extracted from E. coli using the Presto ${ }^{\mathrm{TM}}$ Mini Plasmid Kit Cat. Nos. PDH300 (Geneaid).

\subsection{Mammalian Cell Culture}

\section{Tissue Culture Media - RPMI}

Cells were cultured with RPMI 1640 media (Gibco, ThermoFisher) (SKU \# 11875-093) with 2 $\mathrm{mM}$ L-glutamine and $13 \mu \mathrm{M}$ Phenol Red added. RPMI was supplemented with $10 \%$ (final concentration) heat-inactivated foetal calf serum (FCS) unless stated otherwise.

RAW 267.4 cells and THP-1 cells were cultured for use in live cell experiments. HeLa cells were cultured for use in plasmid transfection and expression. All mammalian cells were cultured in RPMI media. RPMI and PBS (Gibco Life Technologies) were warmed to $37^{\circ} \mathrm{C}$ 
Chapter 2 Methods

before use unless stated otherwise. Cells were incubated in a cell culture humidified incubator at $37^{\circ} \mathrm{C}$ with $5 \% \mathrm{CO}_{2}$. Cell counting was performed using a haemocytometer, staining cells with trypan blue dye (Sigma Aldrich). All cell culture maintenance was performed within a Laminar Flow Hood using CellStar Cell Culture Flasks. Adherent cells were cultured in treated flasks with filter caps, whereas suspension cells were cultured in untreated flasks with screw caps.

\subsubsection{Adherent Cell Lines}

HeLa cells (Sigma Aldrich) and RAW 264.7 cells (ATCC® TIB71TM) were maintained and treated as follows: Cells were passaged every 3 days, or when they reached $90 \%$ confluency. To collect cells, media was removed, and cells washed with PBS. TrypLE Express Enzyme (ThermoFisher Scientific) was added then the majority aspirated off, leaving a thin film. The flask was then incubated at $37^{\circ} \mathrm{C}$ for 10 minutes then washed repeatedly with $37^{\circ} \mathrm{C} \mathrm{RPMI}$ to resuspend cells. The cell suspension (1/20 volume) was transferred to a new flask with fresh RPMI and incubated. To defrost cells, the frozen ampoule was warmed in a $37^{\circ} \mathrm{C}$ water bath then emptied into a Falcon tube, then $4 \mathrm{~mL}$ RPMI was added. The cells were centrifuged and supernatant containing cryoprotectant was discarded. Cells were resuspended in RPMI and transferred to a flask for incubation. To freeze cells for long term storage, $2.0 \times 10^{6}$ cells were added to $1 \mathrm{~mL}$ RPMI with $10 \%$ DMSO in a cryo vial. Cells were placed in $-80^{\circ} \mathrm{C}$ within a CoolCell ${ }^{\circledR} \mathrm{LX}$ (Biocision) then transferred to liquid nitrogen for long term storage the following day.

\subsubsection{Suspension Cell Lines}

THP-1 cells (ATCC® TIB-202 ${ }^{\mathrm{TM}}$ ), purchased from Sigma-Aldrich, were treated as follows: cells were revived from long term storage by thawing frozen ampule containing cells in a $37^{\circ} \mathrm{C}$ water bath, then transferring cells to a Falcon tube and slowly adding $4 \mathrm{~mL}$ RPMI. Cells were centrifuged at low speed, then supernatant containing cryoprotectant was decanted. The pellet was resuspended in $2 \mathrm{~mL}$ RPMI (20\% FCS), supplemented with $0.05 \mathrm{mM} \beta$-mercaptoethanol and $1 \mathrm{mM}$ pyruvate, then cells were transferred to a $35 \mathrm{~mm}$ culture dish and incubated. Media was changed every $2-3$ days. Further supplemented RPMI was maintained for the first passage before returning to $10 \%$ FCS. THP-1 cells were sub-cultured every 3 days, maintaining cells between $5.0 \times 10^{5}$ cells $/ \mathrm{mL}$ and $1.0 \times 10^{6}$ cells $/ \mathrm{mL}$. THP- 1 cells were sub-cultured 10 times before returning to frozen stocks. THP-1 cells were stimulated with Phorbol 12-myristate 13-acetate (PMA; Sigma Aldrich, P1585) resuspended in DMSO, at a final concentration $5 \mathrm{ng} / \mathrm{mL}$ to terminally differentiate the cells into adherent macrophages. THP-1 cells were stimulated for 
48 hours before PMA and media was removed, replaced with fresh RPMI. To freeze THP-1 cells for long term storage, $3.0 \times 10^{6}$ cells $/ \mathrm{mL}$ were resuspended in freezing media comprised of $90 \%$ FCS and $10 \%$ glycerol within a cryo vial. Cells were placed in $-80^{\circ} \mathrm{C}$ within a CoolCell ${ }^{\circledR}$ and transferred to liquid nitrogen the following day.

\subsection{Plasmid and Strain Construction}

This section describes the workflow to clone pGBKT7 and pGBT9 with BepA1 and BepA2 inserts.

\begin{tabular}{|c|c|}
\hline \multicolumn{2}{|l|}{ pGBKT7-BepA1 } \\
\hline Forward: BepA1_F & $\begin{array}{l}\text { 5'- GCG CAT ATG CTA AAT TAT AAG GAG GTC GTT } \\
\text { ATG -3' }\end{array}$ \\
\hline Reverse: BepA1_R & $\begin{array}{l}\text { 5'- GCG GGA TCC TCA AAC AGA TGT TTC ATA GGC } \\
\text { T -3' }\end{array}$ \\
\hline \multicolumn{2}{|c|}{ pGBKT7-BepA2, pGBT9-BepA2, and pCR®2.1-TOPO®-BepA2 } \\
\hline Forward: BepA2_F & $\begin{array}{l}\text { 5'- GCG GAA TTC ATG AGC CAT ATG AAA AAT ATC } \\
\text { AAA CGC AAT -3' }\end{array}$ \\
\hline Reverse: BepA2_R & $\begin{array}{l}\text { 5'- GCG GAA TCC TTA GCA AAC TAT TTT AGC TTG } \\
\text { CCC }-3 \text { ' }\end{array}$ \\
\hline \multicolumn{2}{|l|}{ pcDNA3.1-BepA1 } \\
\hline $\begin{array}{l}\text { Forward: } \\
\text { BepA_Nhe1_Kozak_F }\end{array}$ & 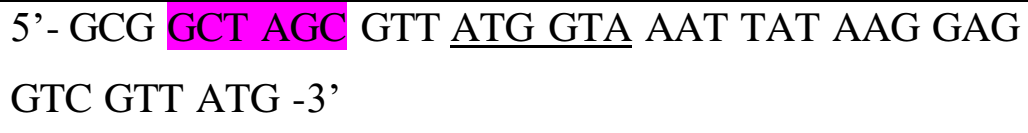 \\
\hline Reverse: BepA1_R & $\begin{array}{l}\text { 5'- GCG GGA TCC TCA AAC AGA TGT TTC ATA GGC } \\
\text { T -3' }\end{array}$ \\
\hline
\end{tabular}

Table 2: Primers Used in Cloning. Engineered restriction sites are highlighted as follows: Green BamHI, Teal - EcoRI, Yellow - NdeI, Purple - NheI. The red highlighted area within the BepA2_R primer was intended to have a BamH1 restriction site (with the sequence GGATCC); however, due to an error in primer creation the incorrect sequence (GAATCC) was included. The underlined sequence within the BepA_Nhe1_Kozak_F primer identifies the Kozak sequence, a ribosomal binding site region essential for transcription within eukaryotic cells.

\subsubsection{PCR}

Q5® High-Fidelity DNA Polymerase (BioLabs) was used to amplify BepA1 and BepA2 inserts from $B$. quintana JK-31 genomic DNA stock, kindly provided by Dr Jane Koehler. PCR assays were performed using the following standard mix: 


\begin{tabular}{|l|l|}
\hline Reagent & Quantity \\
\hline 5X Q5® Reaction Buffer (NEB BioLabs) & $10 \mu \mathrm{L}$ \\
\hline $10 \mathrm{mM}$ dNTPs (NEB BioLabs) & $1 \mu \mathrm{L}$ \\
\hline Forward Primer $(10 \mu \mathrm{M})$ & $2.5 \mu \mathrm{L}$ \\
\hline Reverse Primer $(10 \mu \mathrm{M})$ & $2.5 \mu \mathrm{L}$ \\
\hline Template DNA & $1 \mu \mathrm{L}$ \\
\hline $\begin{array}{l}\text { Q5 High-Fidelity DNA Polymerase (NEB } \\
\text { BioLabs) }\end{array}$ & $0.5 \mu \mathrm{L}$ \\
\hline ddH 20 & $32.5 \mu \mathrm{L}$ \\
\hline Total reaction volume & $50 \mu \mathrm{L}$ \\
\hline
\end{tabular}

Table 3: PCR Reagents. This table shows the quantities and reagents used for PCR amplification of inserts during cloning. Q5 High-Fidelity DNA polymerase was used to ensure accurate amplification of DNA sequences.

Advantage $^{\circledR} 2$ Polymerase in 10X Advantage ${ }^{\circledR} 2$ Polymerase Buffer (Takara Bio USA) was used in circumstances where complete accuracy was not essential.

\begin{tabular}{|c|c|c|c|}
\hline Temperate & Duration & Step & \\
\hline $98^{\circ} \mathrm{C}$ & 30 seconds & Warm up/initial denaturation & \\
\hline $98^{\circ} \mathrm{C}$ & 10 seconds & Denaturation & \multirow{3}{*}{30 cycles } \\
\hline $60^{\circ} \mathrm{C}$ & 30 seconds & Annealing & \\
\hline $72^{\circ} \mathrm{C}$ & 30 seconds & Extension & \\
\hline $72^{\circ} \mathrm{C}$ & 2 minutes & Post-cycle extension & \\
\hline $4^{\circ} \mathrm{C}$ & $\infty$ & Hold until collected & \\
\hline
\end{tabular}

Table 4: PCR cycling parameters. This table shows the PCR cycling parameters used to amply inserts from the $B$. quintana genome for use in cloning. The annealing temperature was dropped to $55^{\circ} \mathrm{C}$ and the extension duration was increased to 1 minute if initial PCR was unsuccessful.

\subsubsection{Gel Electrophoresis}

HyAgarose $^{\mathrm{TM}}$ (HydraGene) was added to $60 \mathrm{~mL}$ TAE Electrophoresis buffer (ThermoFisher), creating a $1 \%$ gel, then microwaved until fully dissolved, swirling when the solution reached boiling. RedSafe ${ }^{\mathrm{TM}}$ Nucleic Acid Staining Solution (iNtRON) was added before pouring the solution into a ThermoFisher gel mould with comb. The agarose was allowed at least 15 minutes to cool and solidify before the comb was removed. TAE Electrophoresis buffer was added to 
the fill line, then $5 \mu \mathrm{L}$ of HyperLadder ${ }^{\mathrm{TM}} 1 \mathrm{~kb}$ (Bioline) was added to the first well. Gel Loading Dye (NEB BioLabs) was added to each sample then $10-20 \mu \mathrm{L}$ sample was loaded into each well. The gel was run at 100 volts for 1 hour, then imaged under UV light using a Typhoon FLA 9500 scanner.

\subsubsection{Restriction Digest}

Plasmid DNA (125 ng) was digested alongside insert DNA with a 1:5 plasmid to insert ratio; the amount of insert DNA varied depending on the size of the insert and plasmid.

I performed double restriction digests using the following mixes:

\begin{tabular}{|l|l|}
\hline pGBKT7 with BepA1 & pGBKT7 with BepA2 \\
\hline NEBuffer 3.1 (BioLabs) & NEBuffer 3.1 (BioLabs) \\
\hline DNA & DNA \\
\hline BamH1 (BioLabs) & BamH1 (BioLabs) \\
\hline Nde1 (BioLabs) & Nde1 (BioLabs) \\
\hline Water & Water \\
\hline
\end{tabular}

\begin{tabular}{|l|l|}
\hline pGBT9 with BepA2 & pcDNA3.1 with BepA1 \\
\hline 10X FastDigest Buffer (ThermoFisher) & 10X Tango Buffer (ThermoFisher) \\
\hline DNA & DNA \\
\hline FastDigest BamHI (ThermoFisher) & BamHI (ThermoFisher) \\
\hline FastDigest EcoRI (ThermoFisher) & NheI (ThermoFisher) \\
\hline Water & Water \\
\hline
\end{tabular}

Table 5: Restriction Digest Reagents. This table displays the different restriction enzymes and buffers used for cloning of different plasmid and insert combinations. All restriction digests were performed in a $20 \mu \mathrm{L}$ reaction volume and all enzymes had $100 \%$ activity in the coupled buffer with no star activity when incubated for 6 hours or less.

Samples were incubated at $37^{\circ} \mathrm{C}$ for 6 hours. Digested plasmid was treated with Calf Intestinal Alkaline Phosphatase (CIAP) (Invitrogen) by adding $1 \mu \mathrm{L}$ and incubating at $37^{\circ} \mathrm{C}$ for 5 minutes. CIAP was heat-inactivated by incubating at $65^{\circ} \mathrm{C}$ for 15 minutes. Following treatment, plasmid and insert DNA was combined using the DNA Clean \& Concentrator (Zymo research), eluting the mixture in $8 \mu \mathrm{L}$. 


\subsubsection{Ligation}

To ligate digested insert DNA into digested plasmid DNA into a circular plasmid, insert and plasmid DNA were purified and concentrated into $8 \mu \mathrm{L}$ for ligation reaction, as follows per tube:

\begin{tabular}{|l|l|}
\hline Reagent & Quantity \\
\hline 10X T4 DNA Ligase Buffer (ThermoFisher) & $1 \mu \mathrm{L}$ \\
\hline T4 DNA Ligase (ThermoFisher) & $1 \mu \mathrm{L}$ \\
\hline Plasmid and Insert DNA & $8 \mu \mathrm{L}$ \\
\hline Total & $10 \mu \mathrm{L}$ \\
\hline
\end{tabular}

Table 6: Ligation Reagents. This table displays the ligase and buffer used for ligation of inserts and plasmids during cloning. All digested insert and digested and CIAP treated plasmid DNA was combined and concentrated into $8 \mu \mathrm{L}$ for ligation.

The following cycling parameters were used:

\begin{tabular}{|l|l|}
\hline Temperature & Duration \\
\hline $10^{\circ} \mathrm{C}$ & 30 seconds \\
\hline $30^{\circ} \mathrm{C}$ & 30 seconds \\
\hline
\end{tabular}

Table 7: Ligation Cycling Parameters. This table shows the cycling parameters for ligation using a Thermocycler.

Thermocycler was run for 16-20 hours.

\subsubsection{Electrocompetent E. coli}

To generate electrocompetent $E$. coli for electroporation with plasmid constructs, a single $E$. coli colony was picked and resuspended in $50 \mathrm{~mL} \mathrm{LB}$ within a flask. The flask was incubated at $37^{\circ} \mathrm{C}$ overnight with shaking at 200 RPM. Two separate flasks containing $250 \mathrm{~mL} \mathrm{LB}$ were inoculated with $5 \mathrm{~mL}$ overnight culture each and incubated at $37^{\circ} \mathrm{C}$ at $200 \mathrm{RPM}$ until $\mathrm{OD}_{600}$ reached $0.3-0.4$ (approximately 2.5 hours). The flasks were immediately transferred to an ice bath and incubated for 15-30 minutes, with occasional swirling. The culture was transferred to ice-cold centrifuge bottles and the cells were pelleted by centrifuging for 15 minutes at 1000 $\mathrm{x} g$ at $4^{\circ} \mathrm{C}$. The cells were washed then centrifuged for 20 minutes at $1000 \mathrm{x} g$ at $4^{\circ} \mathrm{C}$ to pellet. The supernatant was decanted, and the pellets resuspended in $125 \mathrm{~mL}$ ice-cold $10 \%$ glycerol. The bottles were centrifuged for 20 minutes at $1000 \times g$ at $4^{\circ} \mathrm{C}$. The supernatant was carefully 
Chapter 2 Methods

decanted, and the pellets resuspended in $1 \mathrm{~mL}$ ice-cold $10 \%$ glycerol. The cells were aliquoted in $100 \mu \mathrm{L}$ volumes and placed into $-80^{\circ} \mathrm{C}$ for long-term storage.

\subsubsection{Electroporation}

Salts were removed from DNA solutions in preparation for electroporation using drop dialysis, as follows: the DNA solution was placed as a droplet onto a nitrocellulose membrane (MF) suspended on the surface of $\mathrm{ddH}_{2} \mathrm{O}$. After one hour, the drop was pipetted off the filter paper; care was taken not to submerge the droplet. The entire DNA solution was added to a pre-chilled centrifuge tube containing $40 \mu \mathrm{L}$ electrocompetent DH5 $\alpha$ or Top10 cells. The tube was incubated on ice for one minute before loading the solution into a pre-chilled E. coli Pulser® Cuvette with a $0.1 \mathrm{~cm}$ electrode gap (BIO-RAD). The electroporation cuvette was loaded into the pre-chilled E. coli Pulser® (BIO-RAD), then shocked with 1.8 kilovolts. One $\mathrm{mL} 2 \mathrm{X}$ YT media (BD) was immediately added to the electroporation cuvette then the solution was transferred to a fresh centrifuge tube. The cells were incubated at $37^{\circ} \mathrm{C}$ for 1 hour with shaking at 200 RPM. One hundred $\mu \mathrm{L}$ of the cell suspension was spread over an LB plate containing the selection antibiotic, as well as with additional 1/10 dilutions and the remaining cell suspension.

\subsubsection{Sequencing DNA}

Twenty $\mu \mathrm{L}$ plasmid DNA solution was quantified using a NanoDrop spectrophotometer (ThermoFisher Scientific) then sent to Macrogen (South Korea) for Sanger sequencing. Sequence data was analysed using Geneious 9 (Biomatters, New Zealand).

\subsubsection{TOPO Cloning}

BepA2 was cloned into pCR®2.1-TOPO $®$ (Life Technologies) as described by the manufacturer's instructions. To summarise, BepA2 insert was amplified from B. quintana genome using primers BepA2_F and BepA2_R. To achieve cloning, PCR product was combined with water, salt solution, and the TOPO® vector then incubated for 5 minutes at room temperature. Top10 E. coli were transformed using the One Shot ${ }^{\circledR}$ chemical transformation protocol included within the TOPO® TA Cloning® Kit. TOPO cloning reaction was added to chemically competent Top10 E. coli, then cells were heat shocked and plated on LB agar with $\mathrm{X}$-gal and ampicillin. Colonies will turn blue in the presence of X-gal if they contain a plasmid with a functional LacZ gene. The LacZ gene is disrupted because of a successful insert, 
Chapter 2 Methods

therefore white colonies were selected for screening. pCR®2.1-TOPO® contains an ampicillin resistance cassette to select for successful clones. Ten white colonies were screened.

\subsection{Bartonella Phagocytosis and Intracellular Survival}

\subsubsection{Gentamicin Protection Assay of Adherent Phagocytes}

The experiments were carried out essentially as described, with variations from this protocol noted in the Results text.

THP-1 or RAW 267.4 cells were seeded into 24 -well plates at $2.5 \times 10^{5}$ cells per well, in $1 \mathrm{~mL}$ media. Plates were incubated for 24 hours to allow cells to adhere and reach confluence. Once cells reached confluence, the media was removed from wells and cells were washed three times with $1 \mathrm{~mL}$ warm PBS. Fresh confluent plates of Bartonella were scraped and resuspended in RPMI. The concentration of bacteria was estimated by spectrophotometry. Dilutions of the Bartonella suspension were added to each well with $1 \mathrm{~mL}$ fresh RPMI. The initial bacterial suspension was serially diluted and enumerated on chocolate agar plates. Colonies were later counted to determine a more accurate multiplicity of infection and uptake efficiency of the bacteria. Tissue culture plates were co-incubated for 3 hours, allowing for uptake of bacteria by macrophages._Following the initial incubation period, the cell culture media, along with any extracellular bacteria, was removed from the wells and discarded. To remove as many adherent extracellular bacteria as possible, cells were washed three times with $1 \mathrm{~mL}$ PBS. All remaining extracellular bacteria were killed by adding $100 \mu \mathrm{L}$ of $2500 \mu \mathrm{g} / \mathrm{mL}$ gentamicin (final concentration $250 \mu \mathrm{g} / \mathrm{mL}$ ) and $900 \mu \mathrm{L}$ RPMI to each well, then incubating for 1 hour. Following incubation, the media was removed from the wells and cells were washed three times with 1 $\mathrm{mL}$ PBS, removing gentamicin. Wells were then lysed or further incubated before lysing. Viable intracellular bacteria were harvested from the wells at various time points by removing the media, washing the cells three times with $1 \mathrm{~mL}$ PBS, then adding $500 \mu \mathrm{L} 1 \%$ saponin (Sigma-Aldrich) in PBS. Plates were placed onto an orbital rocker set to 130 RPM for 10 minutes, then vigorously pipetted to ensure complete lysis of macrophages. One hundred microliters of lysate were vortexed then pipetted on a CBA plate. Serial dilutions were made and spread on CBA plates. Plates were incubated until visible colonies formed. Biological replicates were averaged when calculating the adjusted CFU. Plates with $<10$ colonies or $>300$ colonies were recorded but excluded from the final calculations. 


\subsubsection{Bacterial Uptake Kinetics of B. quintana}

THP-1 cells were stimulated with PMA and seeded into 24 -well plates $2.5 \times 10^{5}$ cells per well, in $1 \mathrm{~mL}$ media. A suspension of B. quintana $\left(\mathrm{OD}_{600}\right.$ of 0.002$)$ was prepared and added to the phagocytes at staggered time points for a target $\mathrm{MOI}=10$. Cells were incubated for various periods of time, then washed. Gentamicin was added to all wells and incubated for 1 hour. The gentamicin was removed by multiple PBS washes then all wells were lysed with $1 \%$ saponin. The lysates were serially diluted and plated on chocolate agar to enumerate viable colony forming units. Colonies were counted after 13 days.

\subsubsection{Gentamicin Sensitivity of B. quintana}

To determine the optimal time required for extracellular bacterial killing, a suspension of $B$. quintana was prepared and diluted to $\mathrm{OD}_{600}$ of 0.002 . Nine hundred microliters of $B$. quintana suspension were added to microcentrifuge tubes along with $100 \mu \mathrm{L}$ of gentamicin at a concentration of $2500 \mu \mathrm{g} / \mathrm{mL}$ (final concentration $250 \mu \mathrm{g} / \mathrm{mL}$ ). The microcentrifuge tubes were vortexed and placed in an incubator at $37^{\circ} \mathrm{C}$ for various amounts of time. The contents of the microcentrifuge tubes were vortexed and spread onto chocolate agar plates and left for 13 days before counting. The number of viable bacteria per tube was enumerated through colony counting from direct bacterial suspension, allowing calculation of the percentage of viable bacteria before and after gentamicin treatments of varying durations.

\subsection{Yeast Two-Hybrid Screen for Host Cell Targets of BepA1}

\subsubsection{Yeast Culture}

Y2HGold and Y187 yeast strains were supplied in the Matchmaker® Gold Yeast Two-Hybrid System Cat. Nos. 630489 (Takara Bio USA). Yeast were revived from frozen cultures and plated on YPDA media at $30^{\circ} \mathrm{C}$ for 3-4 days. Media sachets from the Matchmaker ${ }^{\circledR}$ Gold Yeast Two-Hybrid System Cat. Nos. 630489 (Takara Bio USA) were assembled as instructed; media packs were added to $500 \mathrm{~mL} \mathrm{ddH}_{2} \mathrm{O}$ and autoclaved for 15 minutes at $121^{\circ} \mathrm{C}, 15 \mathrm{psi}$. Due to deficiency in the supplied media, some plates were supplemented with $0.16 \mathrm{~g}$ Adenine Sulfate (Formedium), per Litre of media. For long term storage, a single fresh colony was scraped from a plate and resuspended in $300 \mu \mathrm{L} 80 \%$ glycerol $+700 \mu \mathrm{L}$ YPDA broth in a $1.5 \mathrm{~mm}$ cryo vial (Tarsons). 


\subsubsection{Yeast Two-Hybrid System}

Yeast two-hybrid assay carried out as described by the Matchmaker ${ }^{\circledR}$ Gold Yeast Two-Hybrid System User Manual Cat. Nos. 630489 (Takara Bio USA). Plasmid containing bait insert was transformed into haploid, chemically competent yeast. Yeast was prepared as described by the Yeastmaker $^{\mathrm{TM}}$ Yeast Transformation System 2 User Manual Cat. Nos. 630439 (Takara Bio USA), section VI. A. The yeast cells were transformed as described by the Yeastmaker ${ }^{\mathrm{TM}}$ Yeast Transformation System 2 User Manual Cat. Nos. 630439 (Takara Bio USA), section VI. B-C. Haploid yeast containing bait plasmid were mated with haploid yeast containing the Mate and Plate normalised universal human cDNA library of prey proteins. Diploid yeast containing both bait and prey proteins were screened on selective media, interacting bait and prey proteins activating reporters allowing survival. Surviving yeast colonies were recovered, frozen for long-term storage, and the prey plasmid was recovered for identification of interacting library protein. Plasmids extracted from yeast as described by the Easy Yeast Plasmid Isolation Kit User Manual Cat. Nos. 630467 (Takara Bio USA).

\subsection{Transfection of HeLa Cells with BepA1}

To assess transfection efficiency, pcDNA3.1-GFP-yopJ-catmut (see Table 1: Strains and Plasmids) was transfected as a positive control as it expresses GFP and is easily visualised and is approximately the same size as pcDNA3.1-BepA1. Transfection efficiency was assessed by fluorescence microscopy; maximum expression was observed 48 hours following transfection with a 3:1 Viafect (Promega) to DNA ratio. When transfecting in 24-well plates the Viafect solution was prepared by adding $0.5 \mu \mathrm{g}$ DNA and $1.5 \mu \mathrm{L}$ Viafect to $18 \mu \mathrm{L}$ Opti-MEM media, per well. When transfecting into 6-well plates, Viafect solution was prepared by adding $1.5 \mu \mathrm{g}$ DNA and $4.5 \mu \mathrm{L}$ Viafect to $194 \mu \mathrm{L}$ Opti-MEM media, per well. Solutions were incubated at room temperature for 10 minutes before carefully dripping onto wells. Twenty $\mu \mathrm{L}$ of this solution was added to each well of a 24 -well plate, or $200 \mu \mathrm{L}$ to each well of a 6-well plate. Untransfected HeLa cells were used as a negative control alongside transfected HeLa cells.

\subsection{Co-Immunoprecipitation}

HeLa cells were lysed in a non-denaturing lysis buffer with protease inhibitors. Lysates were vortexed for 2 hours at room temperature then centrifuged for 3 minutes at $10,000 \times \mathrm{g}$. Supernatants containing protein were transferred to fresh microcentrifuge tubes. The protein concentration was assessed using a Qubit Protein Assay Kit (ThermoFisher). Ten mg of lysates from both non-transfected and transiently transfected HeLa cells with pcDNA3.1 with BepA1 
were used. The lysates were added to centrifuge tubes, then brought to $500 \mu \mathrm{L}$ with nondenaturing lysis buffer. One $\mu \mathrm{g}$ rabbit anti-MYOZAP antibody (Sigma Aldrich) was added to the samples, which were incubated for 1 hour at $4^{\circ} \mathrm{C}$. Fifty $\mu \mathrm{L}$ homogenised SureBeads ${ }^{\mathrm{TM}}$ Protein A Magnetic beads (Bio-Rad) were added before incubating samples overnight at $4{ }^{\circ} \mathrm{C}$ with end-over-end rotation to prevent settling. Beads were washed twice by using the magnet to draw beads to the side of the tube and adding non-denaturing lysis buffer, then resuspending beads by inverting tubes. Beads were then washed with PBS in the same manner. PBS was removed, and beads were resuspended in $35 \mu \mathrm{L}$ Tris-Glycine SDS $2 X$ Sample Buffer (Novex) before the samples were incubated at $95^{\circ} \mathrm{C}$ for 5 minutes. The beads were removed by magnet and the supernatant containing the antibody-protein complex was transferred to a fresh centrifuge tube.

\subsubsection{Western Blotting}

Novex $^{\mathrm{TM}}$ WedgeWell ${ }^{\mathrm{TM}}$ 10-20\% Tris-Glycine Gel was fitted into a protein electrophoresis gel tank. Running buffer was poured into the central tank until the buffer reached the fill line. Five $\mu \mathrm{L}$ PageRuler ${ }^{\mathrm{TM}}$ Protein Ladder (ThermoFisher), untransfected, and pcDNA3.1-BepA1 transfected protein samples, prepared during the co-immunoprecipitation, were loaded into following wells. In addition, HeLa lysate of untransfected and pcDNA3.1-BepA1 transiently transfected cells was loaded as a control. The tank was attached to a BIO-RAD Powerpac 3000 and run at 160 volts for 30 minutes. The protein was transferred from the gel to a Biodyne ${ }^{\circledR}$ Pre-Cut Modified Nylon Membrane (ThermoFisher) using a transfer block and tank. The gel and membrane were loaded into a sandwich complex and inserted into a transfer block, then placed with ice into a tank, filled with transfer buffer. The transfer tank was surrounded in ice and run overnight at $4^{\circ} \mathrm{C}$ at $300 \mathrm{~mA}$. The membrane was retrieved and dipped protein side down in Ponceau Stain then placed in PBS-T on a rocker for 3 minutes. Membrane was placed in Blocker ${ }^{\mathrm{TM}}$ BSA 5\% (ThermoFisher) on a rocker for 1 hour at room temperature. The blocker was removed and the primary antibody, anti-Myc tag (Abcam) was diluted to 1 in 1,000 in BSA $5 \%$, added to the membrane, then incubated overnight on the rocker at $4^{\circ} \mathrm{C}$. BSA-antibody mixture was removed, and the membrane washed in PBS-T for 5 minutes, repeating for a total of 3 washes. PBS-T was removed and the HRP-conjugated secondary antibody (Abcam) was added at a 1:7000 dilution in BSA 5\% and incubated at room temperature for 1 hour, then washed 3 times in PBS-T, as before. PBS-T was removed and $500 \mu \mathrm{L}$ of Clarity Max ${ }^{\mathrm{TM}}$ Western ECL Substrate was dripped over the membrane before imaging using an Amersham Imager 600 . 


\subsection{Phenotypic Assays of Transfected Cells}

\subsubsection{Cell Migration Assay}

A total of $1.0 \times 10^{4} \mathrm{HeLa}$ cells were seeded into each well of a 24 -well plate. HeLa cells were transfected once they reached $80 \%$ confluence. Ten wells were transfected with pcDNA3.1 with BepA1 and another 10 wells with pcDNA3.1 with no insert. The remaining 4 wells remained untransfected. After 48 hours a single vertical scratch was made in each well in a uniform manner using a sterile pipette tip. The centre of each well was photographed, and the exclusion zone area created by the scratch was measured. The plate was incubated at $37^{\circ} \mathrm{C}$ and the same area in each well was photographed and measured at: 4, $8,12,22$, and 28 hours using the image processing software ImageJ and the macro MRI Wound Healing Tool.

\subsubsection{Enzyme-Linked Immunosorbent Assay}

Transfected HeLa cells were seeded into 6 -well plates at a concentration of $1.0 \times 10^{5}$ then incubated at $37^{\circ} \mathrm{C}$ overnight to allow adherence. Untransfected HeLa cells and HeLa cells transfected with an empty pcDNA3.1 plasmid were included as controls. The supernatant was collected, then $1 \mathrm{~mL}$ fresh $37^{\circ} \mathrm{C}$ RPMI with TNF- $\alpha$ (final concentration of $100 \mathrm{ng} / \mathrm{mL}$ ) was added. After 24 hours, the supernatant was collected. Quantikine® Human IL-6 and Quantikine ${ }^{\circledR}$ Human IL-8 ELISA Kits (R\&D Systems) were used to assess cytokine levels in supernatants. Supernatants were diluted 1/50 to measure cytokine levels within the linear portion of the provided standard curve. Using an EnSpire Plate Reader, OD $_{540}$ was measured and subtracted from $\mathrm{OD}_{450}$ readings to account for imperfections in plate plastic. Excel was used account for supernatant dilutions and subtract baseline measurements. GraphPad Prism was used to determine statistical significance via one-way ANOVA. 


\section{Chapter 3: Interactions Between Bartonella and Macrophages}

\subsection{Introduction}

B. quintana infection begins when an infected body louse feeds on a host. During the blood meal the louse deposits bacteria in excreta at the site of feeding, which get introduced into the dermis by scratching or rubbing. In the skin, the bacteria encounter immune cells such as macrophages, resident within the tissue awaiting foreign invaders. The bacteria avoid clearance by innate immune cells, moving into the lymphatic system and eventually the bloodstream, causing disease. Innate immune cells within the skin are the first line of defence during introduction of Bartonella; understanding the interactions between the bacteria and host cells may better explain the survival of Bartonella and subsequent ability to cause disease in the host. Improved understanding of these interactions may allow for better detection, diagnosis, and treatment. The interactions between $B$. quintana and THP-1 cells, a human macrophage-like cell line, are the focus of this chapter. This is a novel combination representing the interactions between $B$. quintana and the macrophages present in the skin of humans. Of the published studies investigating Bartonella interactions with immune cells, none have characterised the interactions between B. quintana and the immune cells of its reservoir host.

This chapter aims to answer the following questions: Is $B$. quintana internalised by THP-1 cells? Can B. quintana persist or replicate within these macrophages? Will B. quintana escape macrophages after internalisation? To answer these questions, a gentamicin protection assay was developed, allowing for accurate quantification of viable intracellular bacteria. To summarise this protocol: bacteria were incubated with host macrophages, allowing bacteria to become internalised. Extracellular bacteria were killed using the antibiotic gentamicin, which is not internalised into host cells, then macrophages are lysed and remaining viable intracellular bacteria are counted. A gentamicin protection assay is well suited to answer these questions as it can accurately assess bacterial count with no estimation of expected count required. This is particularly useful in this case as this is a novel bacteria-host cell combination. These experiments aim to fill the gap in our understanding of the early interactions between $B$. quintana and the human innate immune system. Other Bartonella spp. have been demonstrated to enter and persist within macrophage cells for up to 24 hours ${ }^{100}$. 
Chapter 3: Interactions Between Bartonella and Macrophages

To develop the gentamicin protection assay protocol the first step was to reproduce the results reported by Musso et al. 2001 ${ }^{100}$. The murine RAW 267.4 macrophage and THP-1 human monocyte cell lines were used, rather than the J774 mouse macrophage cell line. RAW 267.4 cells are a reliable, well-characterised, fast-growing cell line, making them useful in the optimisation of the gentamicin protection assay protocol. By then extending our studies to THP1 cells, the results better represent $B$. quintana infection in humans. We carried out pilot experiments based on those described by Musso et al. with $B$. henselae and RAW macrophages to optimise the gentamicin protection assay protocol before expanding to B. quintana and human cell lines. One weakness of the gentamicin protection assay is it cannot discern the mechanism in which bacteria enter host cells, only if bacteria are intracellular. We hypothesised that B. quintana enter THP-1 macrophages, either via macrophage-mediated phagocytosis or by another mechanism. Once internalised, we expected B. quintana to persist for at least 24 hours within THP-1 cells, utilising immune evasion mechanisms to survive. Musso et al. demonstrated B. henselae can enter J774 macrophages within 30 minutes of co-culturing, reaching a plateau at 160 minutes. They also showed that $B$. henselae can persist intracellularly for 8 hours without a significant decrease in viability, before decreasing in viability over the following 24-hour period ${ }^{100}$. A separate study revealed $B$. henselae survive intracellularly in this manner by preventing fusion of the lysosome with the phagosome ${ }^{56}$. Musso et al. identified the optimal time to co-incubate B. henselae and macrophages was three hours, resulting in the highest number of viable intracellular bacteria; this incubation time was subsequently used for our experiments with $B$. henselae. RAW 267.4 and THP-1 cells were co-incubated with $B$. henselae, then treated with gentamicin to kill all extracellular bacteria, leaving viable intracellular bacteria. Gentamicin was removed, and cells were incubated for varying durations, before lysing macrophages and enumerating intracellular bacteria on plates.

\subsection{Intracellular Survival of $B$. henselae in Murine Macrophages}

The aim of this experiment was to establish the degree to which $B$. henselae can persist or replicate intracellularly. The experiment was carried out three times independently; these replicates were labelled A, B, and C, and are summarised in Figure 3-1. 


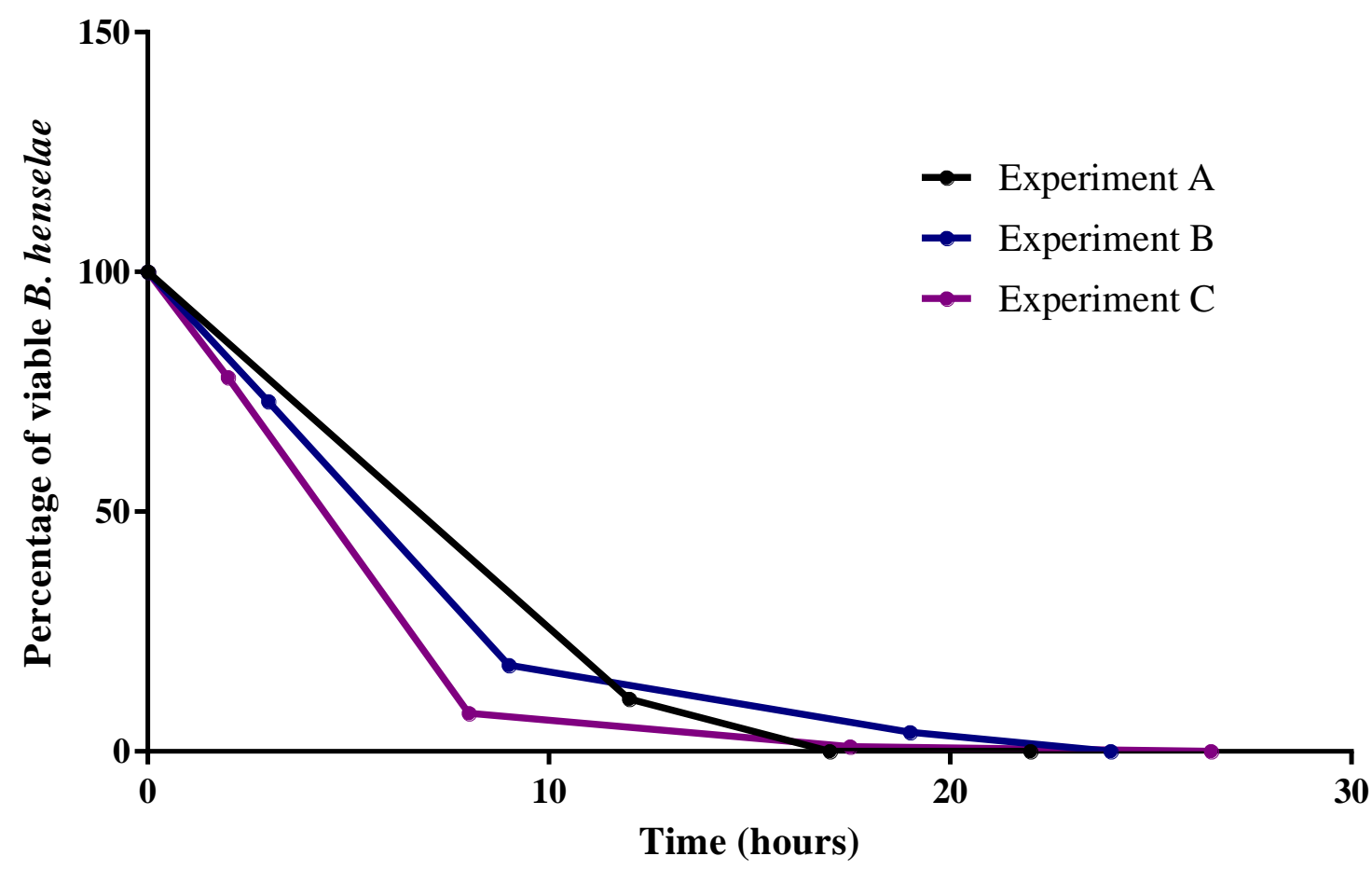

Figure 3-1: Intracellular B. henselae are killed in RAW 267.4 macrophages. RAW 267.4 mouse macrophages were infected with $B$. henselae for three hours, then extracellular bacteria were killed by gentamicin treatment. Intracellular bacteria were quantified over the hours following internalization, to determine the degree to which the bacteria could persist intracellularly. B. henselae was added to the cells at a multiplicity of infection of $0.2-1$. B. henselae are effectively killed within RAW 267.4 cells over a 24-hour period. Each line represents an individual experiment. No statistical analyses were conducted, due to the low number of biological replicates. Results are presented as a percent of the number of viable bacteria recovered from cells that were lysed immediately following removal of gentamicin.

Murine macrophage RAW 267.4 cells killed all intracellular B. henselae within 24 hours in each of the repeated experiments. B. henselae was killed more rapidly by RAW 267.4 macrophages, relative to killing by $\mathrm{J} 774$ cells, as previously demonstrated by Musso et al. Musso et al. also observed a plateau in numbers of viable $B$. henselae for the first 8 hours, suggesting a delay in killing, while under our experimental conditions, B. henselae were killed without any delay following removal of the gentamicin. The data generated from these three experiments (labelled A, B, and C) could not be combined as the number of input bacteria varied significantly, preventing statistical analysis of all three as a singular data set. With a more accurate bacterial quantification method this factor could be eliminated allowing for more accurate repeats between experiments. 


\subsection{Intracellular Survival of $B$. henselae in Human Macrophages}

While we observed immediate and steady declines in numbers of viable $B$. henselae in the presence of murine macrophages, we next aimed to establish if $B$. henselae remain viable intracellularly within human THP-1 monocyte cells. Prior to carrying out the assay, THP-1 cells were terminally differentiated from a suspension monocyte phenotype to an adherent macrophage-like phenotype that approximates the morphology of immune cells in the dermal tissue. To this end, THP-1 cells were treated with PMA for 48 hours prior to addition of $B$. henselae. The THP-1 cells were challenged with B. henselae at an intended multiplicity of infection (MOI) of 100. However, the actual MOI from this experiment was 0.28 , determined by colony counts from bacterial dilution plates.

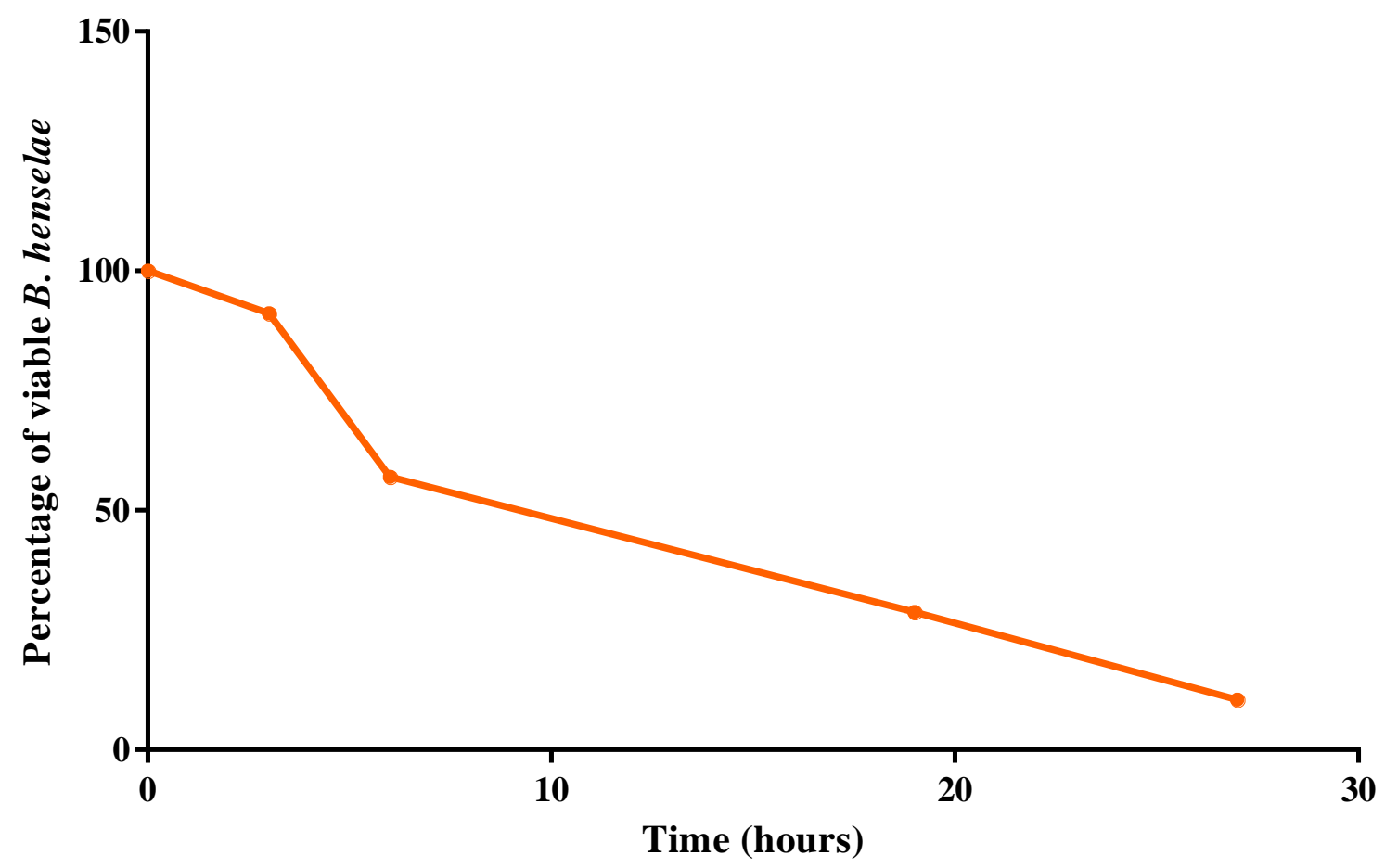

Figure 3-2: Intracellular B. henselae are killed in THP-1 cells. THP-1 cells were infected with B. henselae, then extracellular bacteria were killed by gentamicin treatment. Intracellular bacteria were quantified over the hours following internalization, to determine the rate at which the bacteria could persist intracellularly. $B$. henselae was added to the cells at a multiplicity of infection of 0.28 . Viable intracellular B. henselae are recorded within THP-1 cells for $>27$ hours post gentamicin treatment. The trend suggests a modest reduction in $B$. henselae viability within the first 3 hours post gentamicin treatment.

In contrast with the rapid killing of B. henselae (see Figure 3-1) by murine macrophages, we observed a slower rate of killing and a longer survival time of intracellular bacteria in human macrophages (see Figure 3-2). Viable intracellular B. henselae were detected in THP-1 cells 
Chapter 3: Interactions Between Bartonella and Macrophages

for up to 27 hours. All the results of the $B$. henselae intracellular survival experiments are combined into a single figure (see Figure 3-3) for comparison. The data was also plotted on a semi-logarithmic graph (see Figure 3-4) to visualise emerging trends.

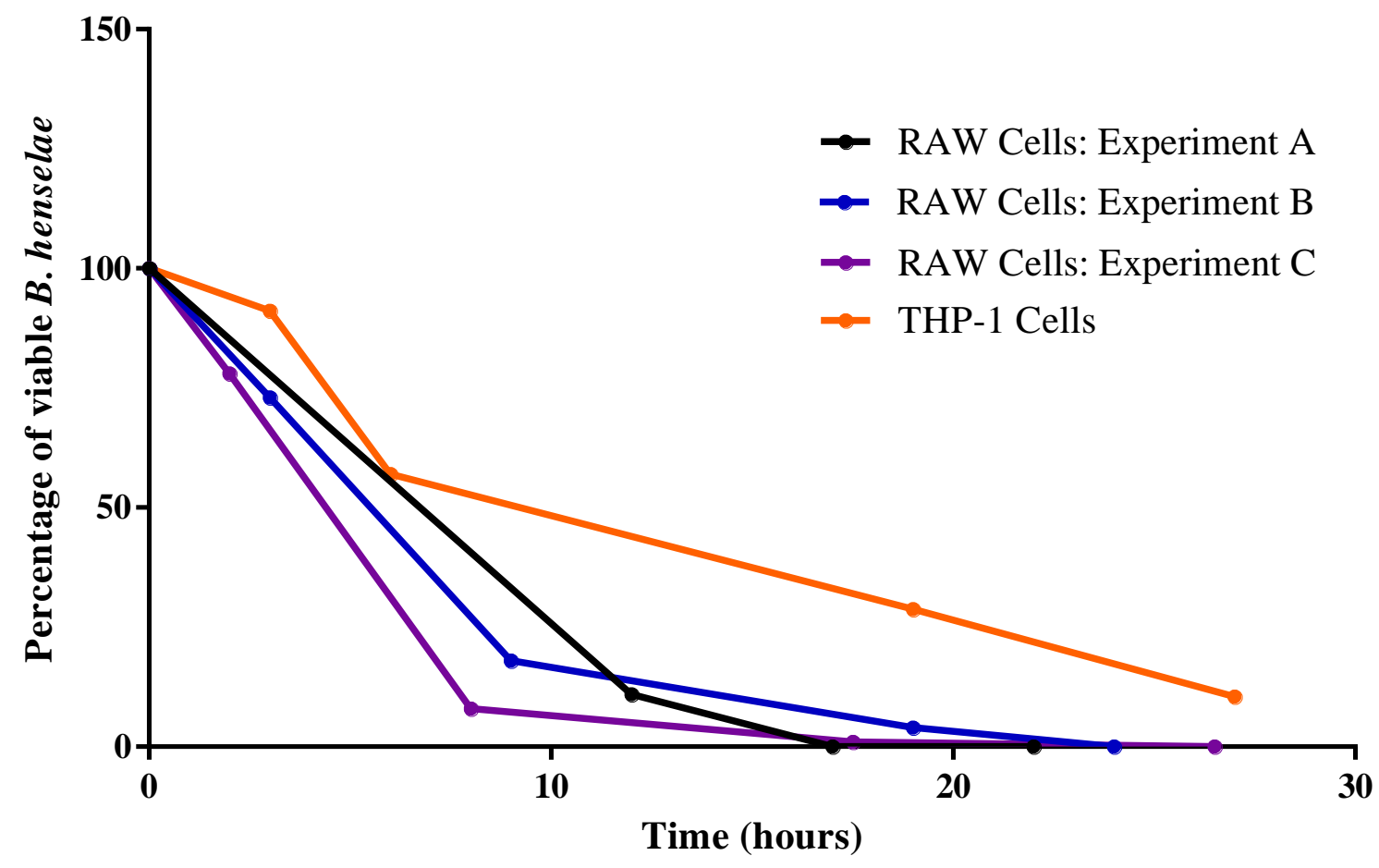

Figure 3-3: Trends in B. henselae killing by RAW 267.4 cells and THP-1s. This is a comparative graph of Figure 3-1 and Figure 3-2, to highlight the difference in trends. Each line of this figure represents an individual pilot experiment. Intracellular bacteria were recovered from phagocytes in the hours following internalization. The trend suggests $B$. henselae killing by RAW 267.4 cells occurs at a higher rate over three experiments compared to THP-1 cells; B. henselae viability drops faster in RAW 267.4 cell experiments, compared to the THP-1 cell experiment. However, statistical analysis cannot confirm this due to low number of biological replicates. 
Chapter 3: Interactions Between Bartonella and Macrophages

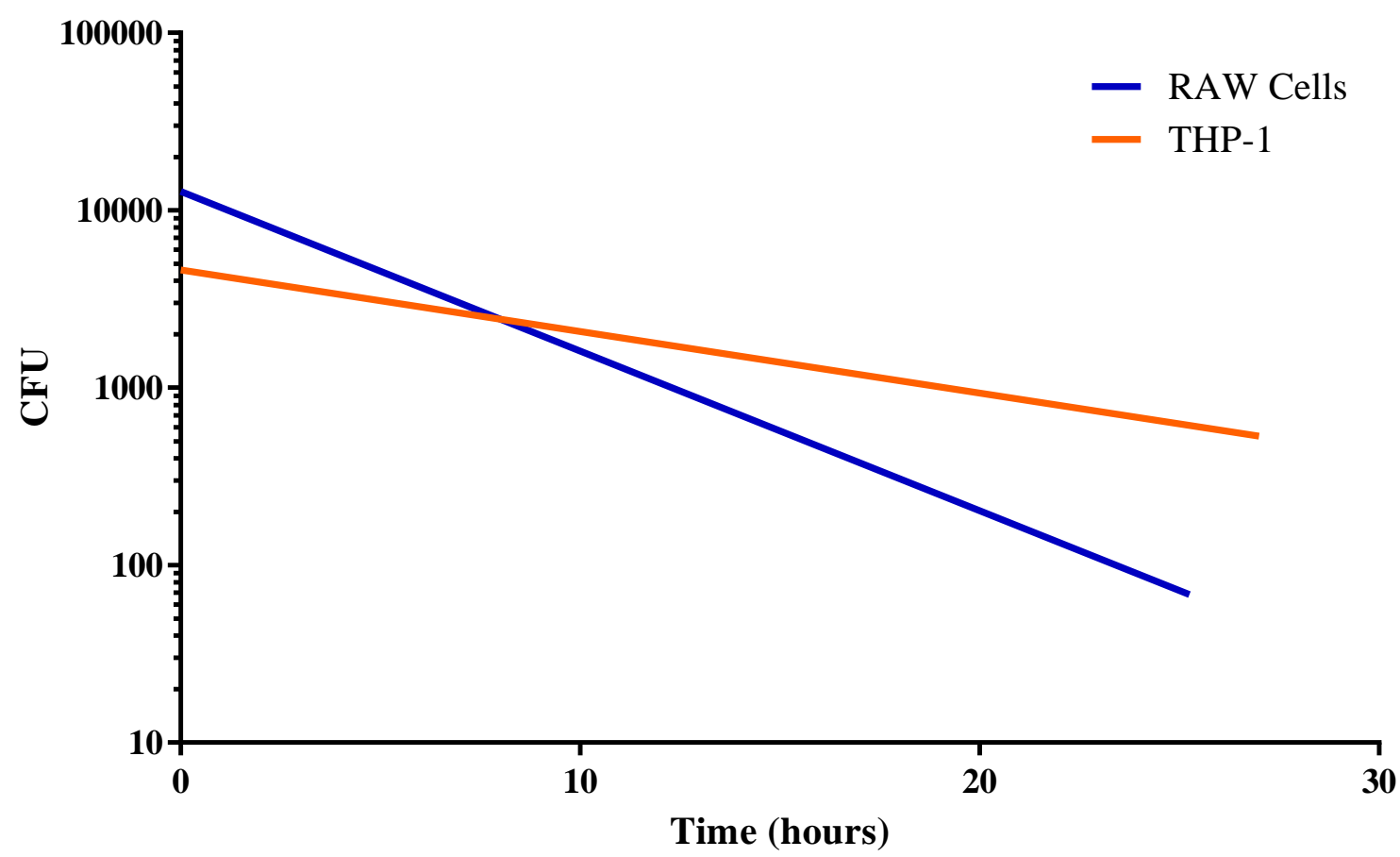

Figure 3-4: Trends in B. henselae killing by RAW 267.4 cells and THP-1 cells on a semi-logarithmic scale. This is a comparative graph of Figure 3-1 and Figure 3-2, with a log y axis to highlight the difference in the rate of intracellular bacteria killing by RAW 267.4 cells and THP-1 cells. Intracellular bacteria were recovered from phagocytes in the hours following internalization. There is a suggestion of a trend, B. henselae appear to be killed slower by THP-1 cells compared to RAW 267.4 cells. RAW 267.4 cell data is a combination of RAW 267.4 cell experimental repeats (see Appendix Figure 1 for the individual experiments).

In addition to intracellular survival within THP-1 cells, viable $B$. henselae was detected in the extracellular medium of THP-1 cells. Extracellular viable $B$. henselae cells, following removal of the gentamicin, were enumerated (see Figure 3-5). Because the gentamicin should have killed all extracellular cells, these bacteria may have escaped the phagocytes. 


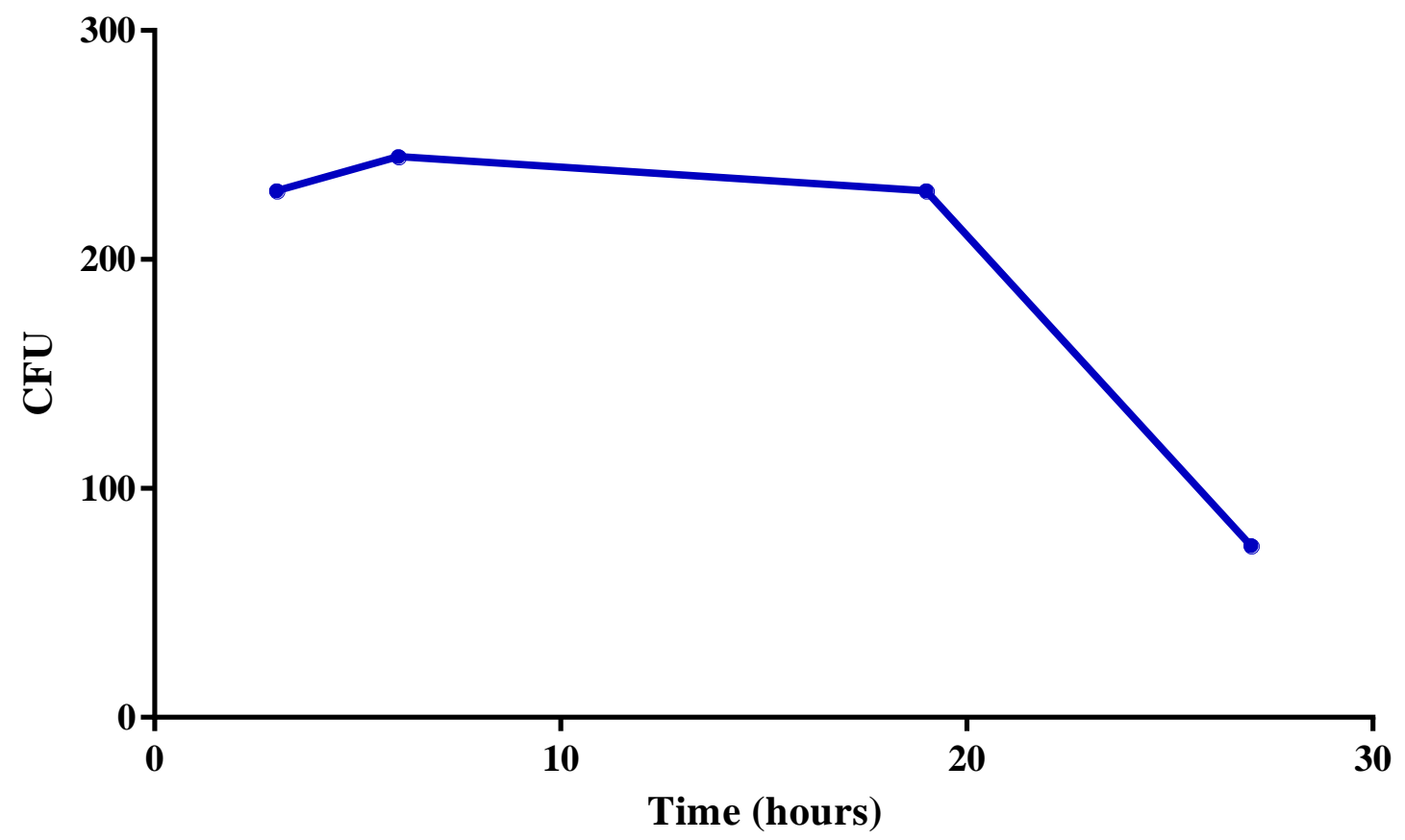

Figure 3-5: Presence of $B$. henselae in THP-1 cell supernatant. THP-1 cells were infected with $B$. henselae, then extracellular bacteria were killed by gentamicin treatment. Media was sampled for viable bacteria during the following hours. For the first 19 hours following gentamicin treatment, a consistent number of viable bacteria were observed in THP-1 cell supernatant. A significant decline in number is then seen for the following 8 hours.

B. henselae may be able to escape THP-1 cells following gentamicin treatment and removal, either due to THP-1 cell death or by escape through the THP-1 cellular membrane. Beginning about 20 hours after gentamicin treatment, the numbers of viable bacteria in media steadily decline; this may be mediated by phagocytosis by THP-1 cells, nutrient deprivation, or a buildup of toxic waste products. Alternatively, there may be a small number of bacteria that survive gentamicin treatment and persist in the media, growing at a similar rate at which bacterial death occurs.

These experiments do not contain error bars or statistical analysis between groups due to few biological replicates. Ideally there would be at least biological triplicate for each data point; however, this was unachievable in these experiments due to a lack of candle jars and incubator space to accommodate the agar plates required to enumerate the bacteria. Each time point required replicates to ensure accurate data and allow for statistical analysis, and dilutions of the cell lysate to ensure at least one dilution was within a countable range. As these experiments using B. henselae, B. quintana, RAW 267.4 cells, and THP-1 cells utilised novel combinations, the expected number of intracellular bacteria could not be estimated. Therefore, many dilutions 
Chapter 3: Interactions Between Bartonella and Macrophages

of cell lysate were used for bacterial counts. Future experiments using these cell combinations could utilise the results from this experiment to estimate the number of intracellular bacteria, allowing them to plate less dilutions and more biological replicates to allow for statistical analysis.

A better approach to investigate Bartonella following a gentamicin protection assay would be to use microscopy techniques to visualise the bacteria. Live/Dead bacterial stains could be used to visualise bacteria during the invasion process, identifying viable and non-viable bacteria. Additionally, intracellular/extracellular staining can be used to visualise intracellular bacteria. These methods could be used to determine the degree to which $B$. henselae remain viable once internalised by phagocytes and determine where the bacteria localise within the host cells. A similar approach was taken by Flannagan et al. in the study of Staphylococcus aureus to determine cellular localisation and viability of intracellular bacteria, the degree of persistence, and escape of the bacteria from phagocytes ${ }^{101}$.

\subsection{Susceptibility of $B$. quintana to Gentamicin}

While $B$. henselae was shown to be rapidly killed by mouse macrophages, and, to a lesser extent, by human macrophages, we were interested in looking at the interactions between a Bartonella species and immune cells from the reservoir host. To this end, we studied the interactions between $B$. quintana and human macrophages. Because little published data describing the interactions between $B$. quintana and immune cells exist, we first carried out pilot experiments. An essential step in the gentamicin protection assay is the killing of all extracellular bacteria by gentamicin treatment. To confirm that B. quintana is sensitive to gentamicin treatment, bacteria were exposed to gentamicin for varying durations, then viable bacteria were enumerated on chocolate agar. Results showed the clear majority of B. quintana are eliminated by 1-hour of gentamicin treatment $(99.999 \%)$ at a concentration $250 \mathrm{ng} / \mathrm{mL}$ (Figure 3-6). Total elimination of B. quintana was seen by 2 hours (i.e., no viable bacteria recovered); therefore, a 2-hour treatment duration was used for further experiments. 


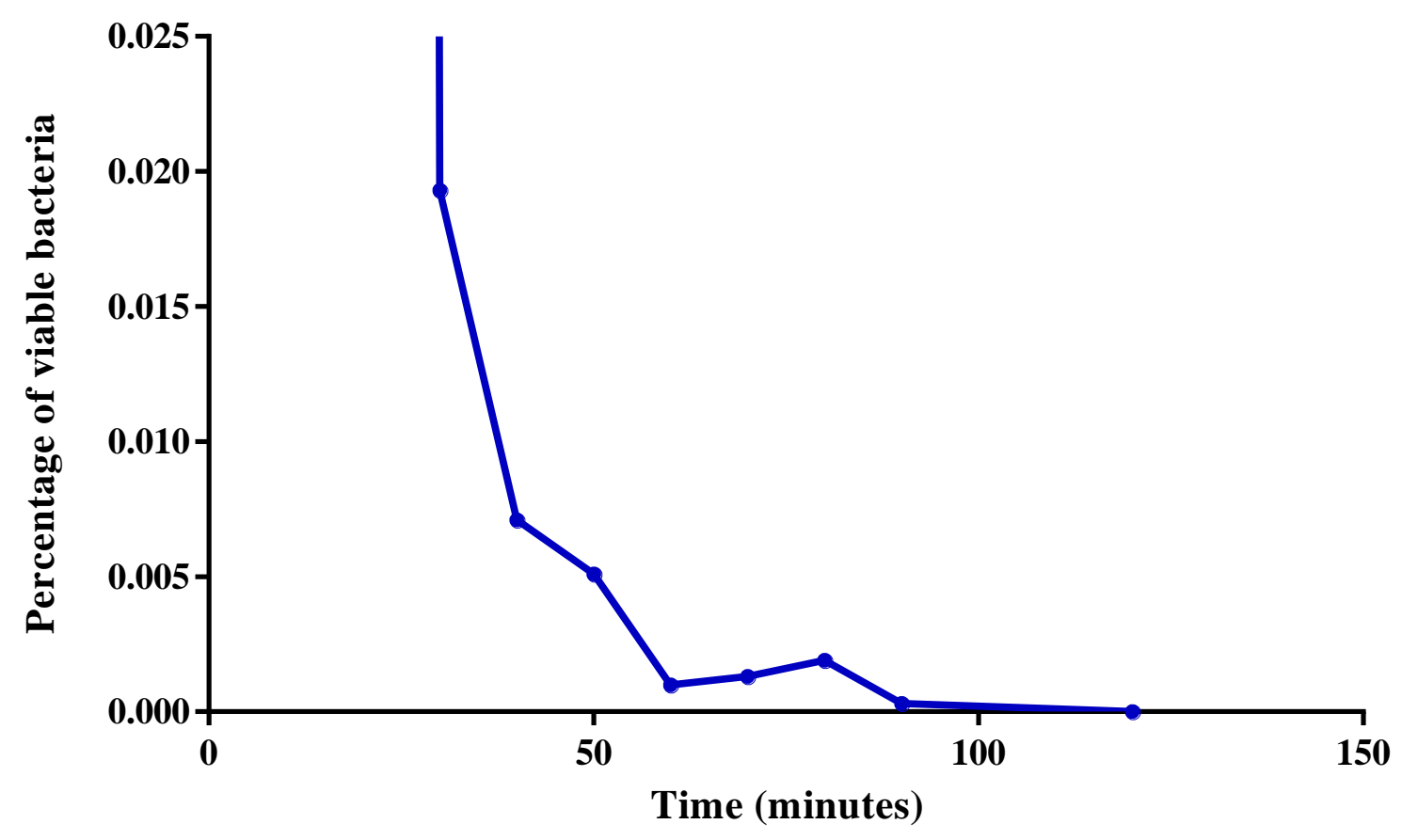

Figure 3-6: B. quintana recovered after exposure gentamicin. This figure shows the effective killing of B. quintana by the antibiotic gentamicin, with $99.999 \%$ of bacteria killed after one hour of treatment; after another hour no viable bacteria could be detected. Bacteria plated after 105 minutes of gentamicin treatment resulted in 320 colonies, indicating 3200 viable bacteria remained $(0.1143 \%$ of the total bacteria before gentamicin treatment). This was unexpectedly high and was possibly due to the formation of large bacterial aggregates that prevent gentamicin killing. However, viable bacteria were not detected thereafter. Graph including outlier in appendix (see Appendix Figure 2).

Our next aim was to determine if $B$. quintana interacted with THP-1 cells similar to B. henselae. With the exchange of $B$. henselae for $B$. quintana we could no longer make the same assumptions about the gentamicin protection assay protocol, based on results described in Musso et al ${ }^{100}$. Specifically, the optimal co-culture time was not assumed to be three hours but was first empirically determined. Bacterial uptake kinetics were tested to determine the optimal co-incubation duration allowing the maximum number of bacteria to become internalized.

\subsection{Kinetics of THP-1 Phagocytosis of B. quintana}

To effectively investigate the kinetics of THP-1 cell phagocytosis of B. quintana, we tested varying co-incubation durations before treating the cells with gentamicin. 


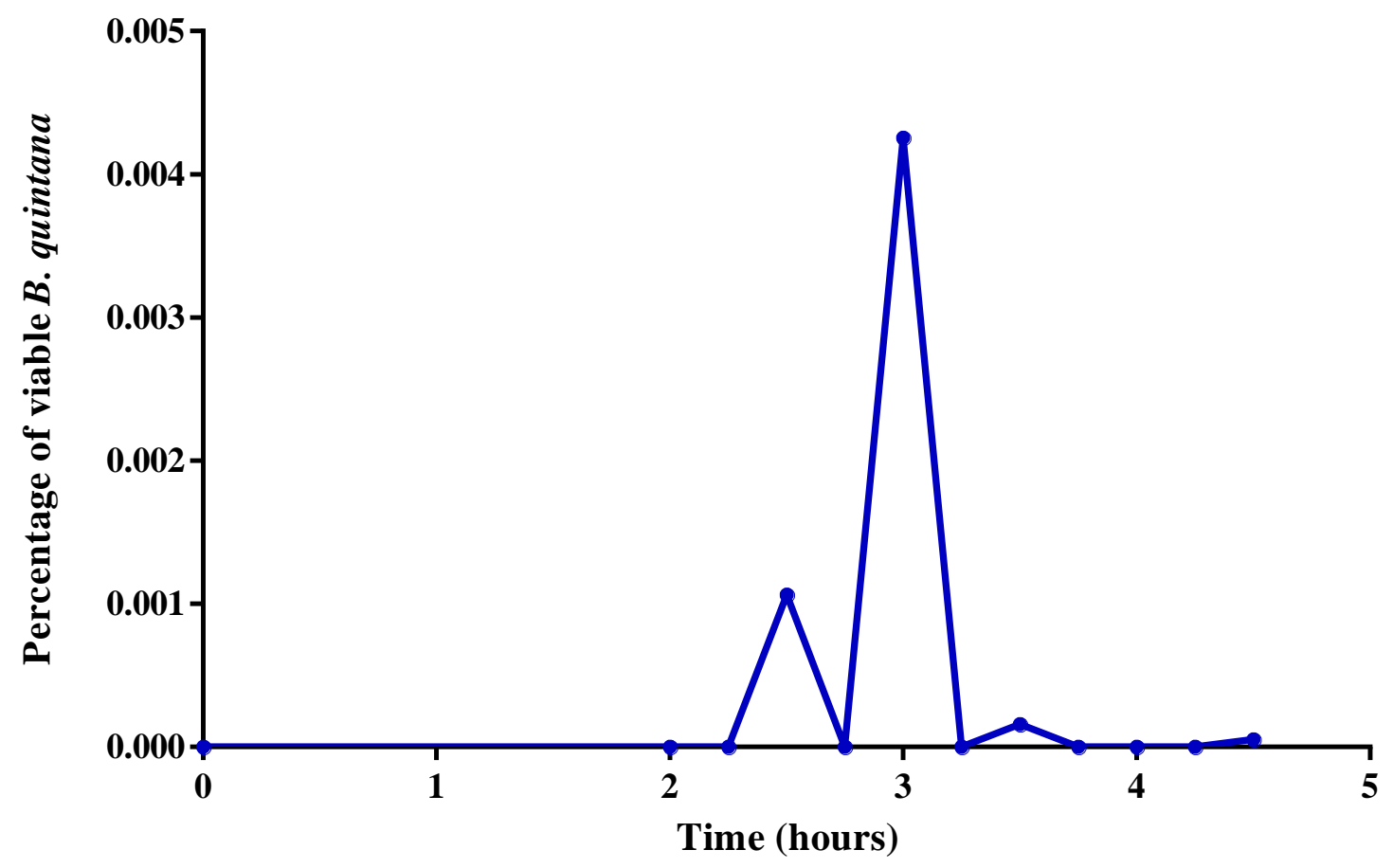

Figure 3-7: B. quintana isolated from THP-1 cells, with varying co-incubation times. This figure shows viable intracellular bacteria recorded following infection of THP-1 cells with B. quintana at an MOI $=0.81$, then killing of extracellular bacteria by gentamicin treatment. The y-axis shows the percentage of the recovered bacteria compared to the number of input bacteria. Little difference was seen between all co-incubation durations showing minimal internalisation of B. quintana. However, a spike in recovered bacteria was observed at 3 hours.

We anticipated seeing high numbers of viable intracellular B. quintana, with a plateau reached at $2-4$ hours, indicating the ideal co-incubation duration. However, strikingly low numbers of viable intracellular bacteria were detected at all the tested co-incubation times (Figure 3-7). This was unexpected, and in contrast to the experiments that showed much higher levels of viable intracellular $B$. henselae in THP-1 cells following a 3-hour co-incubation time.

One explanation for the low number of recovered bacteria is the saponin lysis method reduces viability of $B$. quintana. Saponin has been demonstrated to not reduce viability of gramnegative bacteria ${ }^{102}$; therefore, it is unlikely saponin is the direct cause of low numbers of viable B. quintana recovered. Perhaps lysis of macrophages reduces B. quintana viability, although lysing macrophages using saponin and counting intracellular gram-negative bacteria by plating lysate has been demonstrated previously without a reduction in bacterial viability ${ }^{103}$. To determine if $B$. quintana is sensitive to either of these factors the following experiment could be conducted. One percent saponin or PBS could be added to a known number of viable $B$. 
Chapter 3: Interactions Between Bartonella and Macrophages

quintana and incubated for 15 minutes with rocking, to mimic conditions of macrophage lysis. Bacterial viability could be measured following exposure to saponin to detect toxicity to the bacteria. However, since similarly low numbers were not seen for B. henselae, sensitivity to saponin is unlikely to be the explanation for our results.

Another explanation for the low number of recovered bacteria is that B. quintana inhibits or delays phagocytosis by THP-1 cells. This could occur through several possible mechanisms. First, a small number of B. quintana may get internalised by phagocytosis by THP-1 cells, then inhibit further phagocytosis through the action of Beps, as has been described elsewhere ${ }^{104,105}$. Secondly, B. quintana LPS may be inhibiting phagocytic action by THP-1 cells. Similar effects have been observed in the literature; $B$. quintana LPS acts as a TLR-4 antagonist, inhibiting production of proinflammatory cytokines in human peripheral blood mononuclear cells ${ }^{106}$. It's possible this inhibitory effect is additionally preventing effective phagocytosis of B. quintana by THP-1 cells. Another important note, Hong et al. demonstrated B. tribocorum is poorly phagocytosed by reservoir host, rat macrophages, but does not inhibit their phagocytosis of $E$. coli $^{60}$. This demonstrates Bartonella spp. can avoid phagocytosis by macrophages, without inhibition macrophage phagocytosis. The results of this section are in line with the literature and display the importance of studying the interactions between Bartonella spp. and their reservoir host cells.

The largest peak, at three hours, is an intriguing result; it could result from B. quintana driven inhibition of macrophage phagocytosis, followed by a sudden increase in bacteria uptake at 180 minutes, either by phagocytosis or an alternative invasion mechanism. This peak is possibly an artefact of the experimental method; though it should also be considered this peak is only 400 recovered viable bacteria of the estimated 94,000 added to the THP-1 cells. Therefore, although it appears striking when compared with the other data points, it's still a very low number of recovered bacteria. This result should be confirmed by repeating the experiment.

After the conclusion of this experiment, we experienced laboratory contamination of our $B$. quintana stocks, preventing further optimisation or repetition of these experiments within the available time. If a similar result was observed in repeated experiments, microscopy could be used to visualise the interaction between THP-1 cells and B. quintana. Video microscopy would be particularly useful to characterise the bacteria-THP-1 interaction. This approach would determine if $B$. quintana avoid internalisation or if they are quickly killed following phagocytosis. A protocol originally developed to identify viable intracellular Neisseria 
Chapter 3: Interactions Between Bartonella and Macrophages

gonorrhoeae could be adapted for B. quintana, implementing membrane permeable and impermeable fluorescence dyes to visualise and distinguish between live and dead B. quintana and THP-1 cells ${ }^{107}$. Additionally, contact time between B. quintana and THP-1 cells could be improved in future experiments by spinning plates via centrifuge to facilitate bacteria-host cell contact. As B. quintana are non-motile, this would increase exposure of the bacteria to THP-1 cells, which are adhered to the plate surface. 
Chapter 3: Interactions Between Bartonella and Macrophages

\subsection{Discussion}

\section{Intracellular Survival of B. henselae in Mouse Macrophages}

$B$. henselae internalisation and intracellular persistence in murine macrophages was previously characterised by Musso et al., making it a useful Bartonella spp. for us to use to optimise the gentamicin protection assay ${ }^{100}$. The experiments carried out by Musso et al. used murine $\mathrm{J} 774$ macrophage cells; however, we used murine RAW 267.4 macrophages as they are a readily available, fast growing, well characterised cell line, making them suitable for pilot experiments. We investigated interactions between RAW 267.4 cells and B. henselae and determined, over three experiments, that RAW 267.4 cells effectively cleared intracellular bacteria. The bacterial input of each experiment was subject to variation, likely resulting from varied readings from the spectrophotometer. Despite this experimental variation, similar rates of decline in numbers of viable intracellular $B$. henselae were seen in all cases. These experiments showed $B$. henselae was internalised by RAW 267.4 cells but rapidly killed; all intracellular bacteria were cleared by 24 hours. Musso et al. made the following observation when exposing J774 cells to $B$. henselae at an $\mathrm{MOI}=10$, "up to $8 \mathrm{~h}$ post-infection, the number of viable bacteria inside nonactivated J774 cells remained almost constant" ${ }^{100}$. As both $\mathrm{J} 774$ and RAW 267.4 cells are murine macrophage cell lines, we expected a similar result. However, we found that RAW 267.4 cells were much more effective at killing intracellular $B$. henselae, with immediate reduction in numbers of viable intracellular $B$. henselae observed; by 8 hours, only the remaining viable intracellular bacteria were $8-25 \%$ of the numbers recovered immediately following gentamicin treatment. The intended multiplicity of infection (MOI) of these experiments was 10, i.e., macrophages would be infected with bacteria at a 10:1 ratio. The number of input bacteria was initially estimated using a spectrophotometer at $\mathrm{OD}_{600}$; however, a more accurate quantification of viable bacteria was calculated following each experiment via viable cell counting of bacterial cell suspension, revealing an actual MOI that varied from 0.2 -1 . The discrepancy between viable cell counts and estimates from the spectrophotometer may be because $B$. henselae grows poorly in liquid media and must be harvested from confluent plates, which may include many dead bacterial cells.

To minimise discrepancies between viable cell counting and absorbance readings, $B$. henselae and B. quintana were consistently harvested four days after passaging, when maximum viability was expected. Future experiments could be optimised by quantifying input bacteria using a qPCR method to count genome equivalents. This would allow improved quantification of bacteria before use in the experiment and without the need for absorbance readings. However, quantification of bacterial cell number by amplification of gDNA can include non-viable 
bacteria as the DNA can remain stable for a short time after bacterial cell death. Alternatively, a reverse transcriptase qPCR method could be developed to quantify viable bacteria by amplification of mRNA, which breaks down readily after bacterial cell death.

As expected vs observed MOI differed drastically, a direct comparison between the results obtained by Musso et al. and the results of these B. henselae and mouse macrophage assays could not be made. The low MOI used in our experiments could affect the rate at which RAW 267.4 cells killed the bacteria through phagocytosis and subsequent lysosome fusion. $B$. henselae has been demonstrated to prevent lysosome fusion with the phagosome in murine macrophages ${ }^{56}$; perhaps this activity requires a higher number of intracellular bacteria to be carried out. Alternatively, there may be differences between RAW 267.4 and J774 murine macrophages that are responsible for the differential rate of killing. To obtain more illuminating results a method of quantifying the bacteria to reliably obtain a specific MOI must be achieved, allowing for repeated experiments under the same conditions. Having enough biological replicates to perform statistical analysis of the results is also essential. A qPCR quantification method amplifying a Bartonella-specific marker could be adapted to quantify bacterial genome equivalents (a proxy for bacterial cell number) both prior to addition of the bacteria to the tissue culture cells and used to quantify viable bacteria following phagocytosis. One consideration of qPCR for genome equivalents is gDNA may remain stable for a short time following the death of a cell; therefore, some genomes would be counted from non-viable bacteria. However, gDNA would likely break down fast enough for this to not considerably impact quantification. A RTqPCR method could be implemented, targeting mRNA, which breaks down much faster following cell death. The challenge of this method would be the selection of an mRNA expressed at a constant level between cells and throughout the experiment; for this reason, qPCR for genome equivalents would be the more reliable quantification method. A qPCR quantification method would allow for far more biological replicates, avoiding the logistical considerations surrounding plating for CFU. With the addition of at least three biological replicates and three time points within the first eight hours, standard deviations for each data point could be calculated, along with $\mathrm{p}$ values to compare between time points and groups. We could not conduct these experiments due to contamination of Bartonella stocks and time limitations. Although our experimental results differed from those reported by Musso et al, the assay for intracellular survival of $B$. henselae was next adapted to study the interaction of the bacteria with a human monocyte/macrophage cell line. 
Chapter 3: Interactions Between Bartonella and Macrophages

\section{Intracellular Survival of $\boldsymbol{B}$. henselae in Human Macrophages}

Gentamicin protection assay experiments with B. henselae and murine RAW 267.4 cells revealed rapid killing of $B$. henselae. The next question we asked was if $B$. henselae would have similar interactions with a human macrophage line, THP-1 cells. The reservoir host of $B$. henselae is the cat; therefore, both murine or human macrophages represent cells from incidental $B$. henselae hosts. We hypothesised we would observe similar interactions with $B$. henselae for both THP-1 and RAW 267.4 cells, with rapid internalisation and killing of $B$. henselae. However, we found that THP-1 cells were less effective at clearing intracellular $B$. henselae, compared to RAW 267.4 cells. Repeating this experiment with more biological replicates is required to confirm a statistically significant difference between these two groups . This is an interesting finding as both THP-1 cells and RAW 267.4 cells are derived from incidental hosts; however, there was a clear difference in the interaction, as assessed by the rate of $B$. henselae killing by both cell lines. Some of these differences could be due to artefacts that result from using immortalised cell lines. The mechanisms involved in the slower killing of intracellular B. henselae by THP-1 cells could be further explored. In 2005 Kyme et al. investigated a mechanism of $B$. henselae intracellular survival with $\mathrm{J} 774$ murine macrophages that involved bacterial-driven prevention of lysosomal fusion with the phagosome ${ }^{56}$. Lysosomal fusion with the phagosome could be investigated in a similar manner with RAW 267.4 cells and THP-1 cells to compare.

\section{Intracellular Survival of B. quintana in Human Macrophages}

The goal of our experiments was to study the interactions between B. quintana and immune cells from a reservoir host. There were several reasons for this interest. First, B. quintana is a significant human pathogen but remarkably little is known about its interaction and evasion of the innate immune response. Second, because humans are the reservoir host for B. quintana, the bacteria may have evolved evasion mechanisms specifically for human immune cells, as a result of thousands of years of co-evolution. B. quintana can survive and persist in the bloodstream for extended periods, suggesting likely uncharacterised mechanisms exist for subverting monocyte killing. The related pathogen Bartonella vinsonii has been shown to impair monocyte phagocytic activity against $E$. coli during chronic experimental infection in dogs ${ }^{67}$; We wanted to know whether B. quintana could survive and replicate in phagocytes, and employed a human macrophage cell line, THP-1, to answer this question. Before assessing the degree of intracellular survival, we first investigated bacterial uptake kinetics to determine the optimal co-incubation time, allowing for the maximum number of internalised bacteria. An optimal co-incubation time of three hours was reported by Musso et al., for B. henselae and 
Chapter 3: Interactions Between Bartonella and Macrophages

J774 cells, but we needed to determine this empirically for B. quintana with human macrophage cells. However, an unexpected result was observed, as minimal viable B. quintana were recovered from THP-1 cells at any time point. This result does not distinguish between bacteria resisting engulfment by THP-1 cells, versus the bacteria being engulfed but immediately and efficiently killed, or perhaps a cytotoxic effect on the THP-1 cells, mediated by B. quintana. One explanation for this observation was that internalisation was inhibited by a B. quintana factor, such as a trimeric autotransporter adhesin or the Bep homologue proteins, effector proteins secreted into the cytoplasm of host cells by the Type IV secretion system, though THP1 cells have not yet been identified as a target of the Bartonella T4SS. Possible candidates include the variably-expressed outer membrane proteins (Vomp), B. quintana homologues of the Yersinia trimeric autotransporter adhesin $\mathrm{YadA}^{108}$, which inhibits phagocytosis of the bacteria by leukocytes ${ }^{109}$. B. quintana LPS has demonstrated pro-inflammatory cytokine blocking effects on peripheral blood mononuclear cells ${ }^{106}$, perhaps there are further unexplored effects that are affecting phagocytic activity in THP-1 cells. Additionally, Hong et al. demonstrated B. tribocorum is poorly internalised by human monocytes but does not affect phagocytic activity of these macrophages ${ }^{60}$. B. quintana may have a similar effect on THP-1 cells; although further studies are required.

This experiment could only be performed once, due to contamination issues experienced in the laboratory. To study this question further, it would essential to repeat the experiment, with additional replicates. Persistence of $B$. quintana within THP-1 cells could not be assessed due to the low levels of internalisation of the bacteria. Further researchinto B. quintana intracellular persistence could provide useful information as little is known about the interactions between B. quintana and immune cells. Hong et al.demonstrated B. tribocorum can persist and possibly even replicating within macrophages, although internalisation was minimal ${ }^{60}$. Our original hypothesis that $B$. quintana would be internalised and persist for greater than 24 hours was not correct, though this conclusion is based on only one experiment, and needs to be confirmed. Poor internalisation of B. quintana by THP-1 cells suggests that macrophages do not typically harbour the bacteria during chronic infection, although the interactions in vivo may differ significantly. Though these experiments raised many new questions, logistical complications prevented further exploration using these assays. Instead, we next focused on a secreted $B$. quintana factor that we hypothesised could be playing a role in the inhibition of phagocytosis. 


\section{Chapter 4: BepA1 Protein Interaction Studies}

\subsection{Introduction}

Effector proteins are a diverse group of virulence proteins secreted by pathogenic bacteria that manipulate host cells, typically by modulating host cell signalling ${ }^{110}$. Effector proteins carry out their function by mimicking or covalently modifying host cell proteins ${ }^{111}$. We hypothesised that a $B$. quintana effector protein was influencing THP-1 cells, affecting phagocytosis, following contact with B. quintana, described in Chapter 3. Phagocytosis relies on complex and dynamic reorganisation of the actin cytoskeleton, engulfing pathogens and debris. Given the role phagocytosis plays in bacterial killing it comes as no surprise that numerous bacterial species have evolved mechanisms to modulate phagocytosis, to their benefit. The following are examples of bacterial species that use secreted effector proteins to modulate phagocytosis, or affect the actin cytoskeleton, among their other effects. Yersinia pestis inhibits host cell phagocytosis via a group of secreted effector proteins, known as the Yops, that interfere with immune cell signalling ${ }^{112}$. Vibrio parahaemolyticus secretes an effector that targets the Rho guanosine triphosphatases (GTPases) family of actin regulators, inhibiting their activity through a covalent AMPylation modification mediated by a FIC domain ${ }^{113}$. A Bartonella rochalimae Type IV secreted effector, Bep2, also has a FIC domain, and targets vimentin, an intermediate filament protein of the cytoskeleton ${ }^{98}$.

The five Beps encoded by $B$. quintana are located immediately downstream of the virB locus, which encodes the structural elements that comprise the VirB/D4 T4SS. BepA1 and BepA2 are effector proteins of $B$. quintana that are homologous to the B. henselae effector BepA, sharing $70 \%$ sequence homology (see Appendix Figure 3). In B. quintana, the BepA homologue gained a stop and start codon pair within the gene, splitting it into two separate proteins, BepA1, containing the enzymatic FIC domain, and BepA2, containing the BID domain. The FIC domain-containing effector protein BepA of $B$. henselae has been demonstrated to inhibit apoptosis, although this activity has been shown to be mediated by the BID domain of this protein $^{84}$. Additionally, the FIC domain-containing effector protein Bep2 of Bartonella rochalimae AMPylates the cytoskeletal intermediate filament protein, vimentin ${ }^{98}$. BepA1 also contains an enzymatic FIC domain, though the cellular targets are unknown. As FIC domaincontaining effector proteins of related Bartonella spp. have been shown to modulate the host cell cytoskeleton and interfere with cellular signalling proteins, B. quintana BepA1 may have a similar effect. We hypothesised that BepA1 may be affecting host cell phagocytosis through 
Chapter 4: BepA1 Protein Interaction Studies

actin signalling modification during $B$. quintana infection. The $B$. quintana BepA2 effector has been shown to interact with host cell adenylate cyclase, increasing cellular cAMP levels and inhibiting apoptosis ${ }^{114}$; additionally, BepA1 and BepA2 may interact together to carry out a function. Therefore, host cellular target of BepA2 was also investigated. 

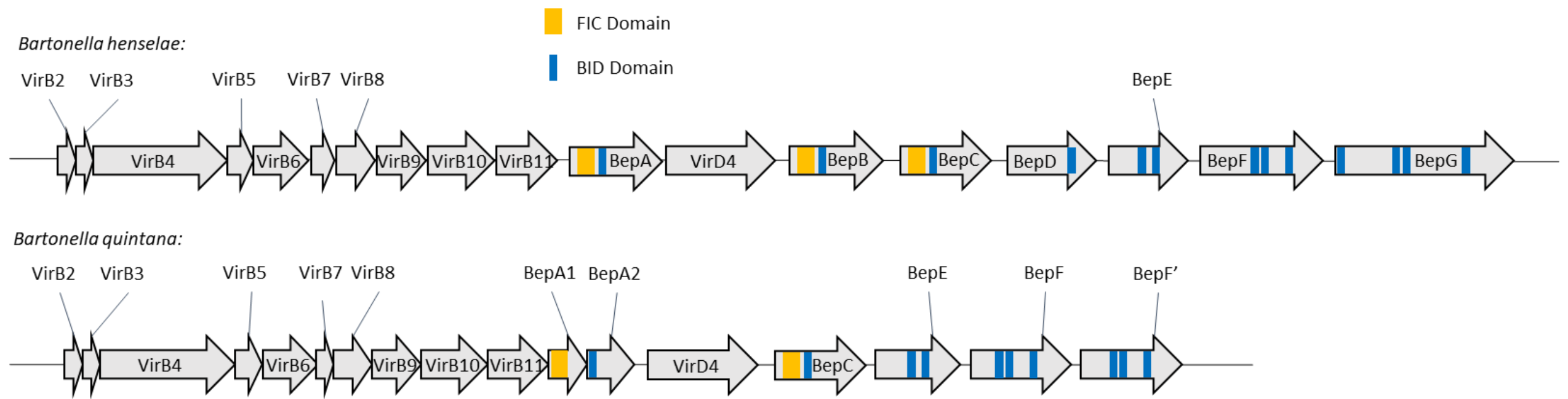

Figure 4-1: Bartonella Effector genes are semi-conserved between B. henselae and B. quintana. Schematic of Bartonella Type IV secretion structural and effector genes. The VirB locus encoding the components of the T4SS is located upstream of the secreted effector Bep genes, with VirD4 located downstream of the BepA homologue. B. henselae encodes seven secreted effectors, whereas B. quintana encodes five. B. henselae BepA contains a FIC and BID domain, while in B. quintana the two domains are separated into two proteins, BepA1, containing a FIC domain, and BepA2, containing a BID domain. The data is derived from the genome sequences of B. henselae str. Houston-1 (NCBI Reference Sequence NC_005956.1) and B. quintana str. Toulouse (NCBI Reference Sequence NC_005955.1). Adapted from a figure titled "VirB Effector Proteins Across Different Bartonella Species", created by Alvey Little. 
The host cell target that is AMPylated by the BepA1 Fic domain is unknown; therefore, the first step we decided to take to determine the involvement of BepA1 in B. quintana infection was to identify eukaryotic cellular targets. We decided to use the yeast two-hybrid assay to identify candidate host cell proteins that interacted with BepA1.

The yeast two-hybrid system is an approach to investigate protein-protein interactions. The protein of interest, i.e., BepA1, referred to as the bait, is coupled to a GAL4 DNA binding domain (GAL4-BD) and expressed in a haploid yeast strain. A cDNA library of eukaryotic host cell proteins, referred to as the prey, was expressed as fusions to a GAL4 activation domain (GAL4-AD) in another haploid yeast strain. When the two haploid yeast strains were mated together, the bait protein had the opportunity to interact with prey proteins. In this case, the associated GAL4-AD and GAL4-BD domains would interact and enable the complex to bind GAL promoters, expressing reporter genes. The bait plasmid has a selection marker allowing yeast to grow on tryptophan deficient media, while the prey plasmid has a selection marker allowing yeast to grow on leucine deficient media. Interacting bait and prey proteins would subsequently activate four independent reporter genes, under different promoters. These promoters are unrelated apart from a shared protein binding site recognised by GAL4-BD. The G1 promoter activates HIS3, the G2 promoter activates ADE2, and the M1 promoter activates AUR1-C and MEL1. Expression of HIS3 and ADE2 allows yeast to synthesize histidine and alanine respectively, enabling growth on deficient media. Expression of AUR1-C confers resistance to aureobasidin-A and MEL1 encodes $\alpha$-galactosidase, turning yeast colonies blue in the presence of $\mathrm{X}-\alpha-\mathrm{Gal}$. The expression of the reporter genes enables both genetic selection and screening of colonies for phenotypic differences. All four of these promoters would be activated following a protein-protein interaction between bait and prey proteins, associating the GAL4-BD and the GAL4-AD. A normalised universal human cDNA library was screened; the appearance of blue colonies on selective media enables isolation, sequencing, and identification of candidate proteins that interact with BepA1.

\subsection{Plasmid Construction}

To carry out a yeast two hybrid screen, we first had to clone the B. quintana bepA1 and bepA2 genes into plasmids to generate translational fusions of each gene with the GAL4 binding domain (GAL4$\mathrm{BD})$. Primers were designed to amplify bepA1 and bepA2, with engineered restriction sites BamHI and NdeI to facilitate cloning into the yeast vector pGBKT7 (see Table 2: Primers Used in Cloning.). Additionally, primers were designed to ensure the GAL4-BD translational fusions would be in frame with the bait genes. Inserts were digested and ligated into pGBKT7, then transformed 
Chapter 4: BepA1 Protein Interaction Studies

into $E$. coli for replication and storage. Plasmids were then extracted and sequenced to ensure correct insertion of bait genes as well as confirming the translational fusion of the GAL4-BD were in frame. These plasmids allow the expression of BepA1 or BepA2 within yeast cells. The bepA1 and bepA2 genes were amplified with primers BepA1_F \& BepA1_R and BepA2_F \& BepA2_R, using a high-fidelity DNA polymerase to minimise point mutations, and a preparation of genomic DNA from B. quintana isolate JK-31 as the template ${ }^{78}$. The resulting PCR products were checked on an agarose gel (see Figure 4-2) to confirm a single product of the correct size was obtained for each reaction. The PCR products were then cloned into the unique BamHI and NdeI restriction sites of the yeast-E. coli shuttle vector, pGBKT7.

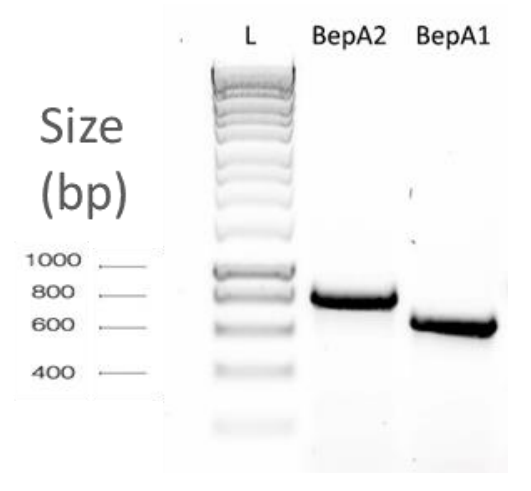

Figure 4-2: BepA1 and BepA2 inserts following PCR. L-Hyperladder 1kb. PCR amplification of inserts for cloning of bepA1 and bepA2 into pGBKT7 for use in the Y2HS. This figure shows amplification of bepAl and bepA2 genes from B. quintana genome, with NdeI and BamHI restriction sites (see Table 2: Primers Used in Cloning.) The size of the bepA2 gene is $834 \mathrm{bp}$ and the bepAl gene is $680 \mathrm{bp}$. Both reactions resulted in bands of the expected size.

Inserts were cloned into pGBKT7 plasmids with NdeI and BamHI restriction sites. Cloning of bepAl was successful (see Figure 4-3: BepAl insert excisedfrom pGBKT7.); however, cloning of bepA2 did not produce successful clones (see Figure 4-4: BepA2 insert screening in $p G B K T 7$.). A band at $680 \mathrm{bp}$ was expected when cloning bepAl and a band of $834 \mathrm{bp}$ was expected when cloning bepA2. 


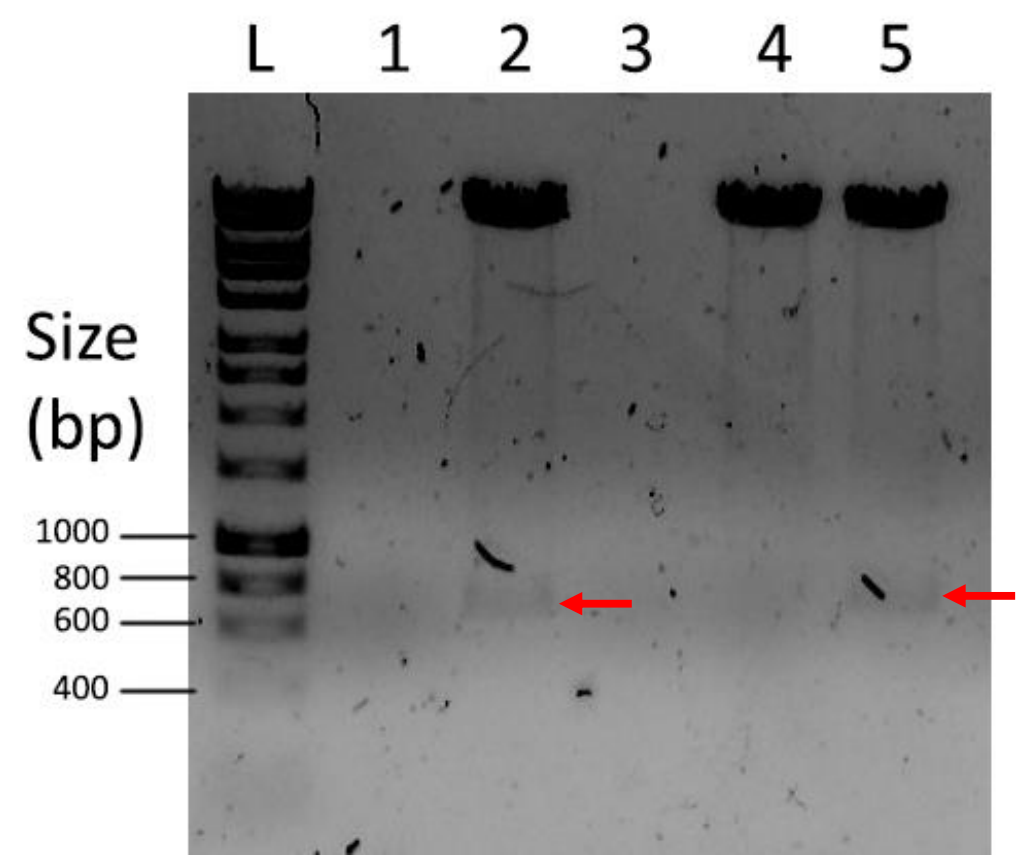

Figure 4-3: BepA1 insert excised from pGBKT7. L - Hyperladder 1kb, 1-5 - screened colonies. In this figure, BepA1 inserts within pGBKT7 were screened via double digest, following cloning. Excised BepA1 can be faintly seen in lanes 2 and 5 (see red arrows). Colonies screened in lanes 1 and 3 did not contain pGBKT7. Colony screened in lane 4 did not contain the BepA1 insert. See Appendix Figure 4 for sequencing data from plasmids in lanes 2 and 5.

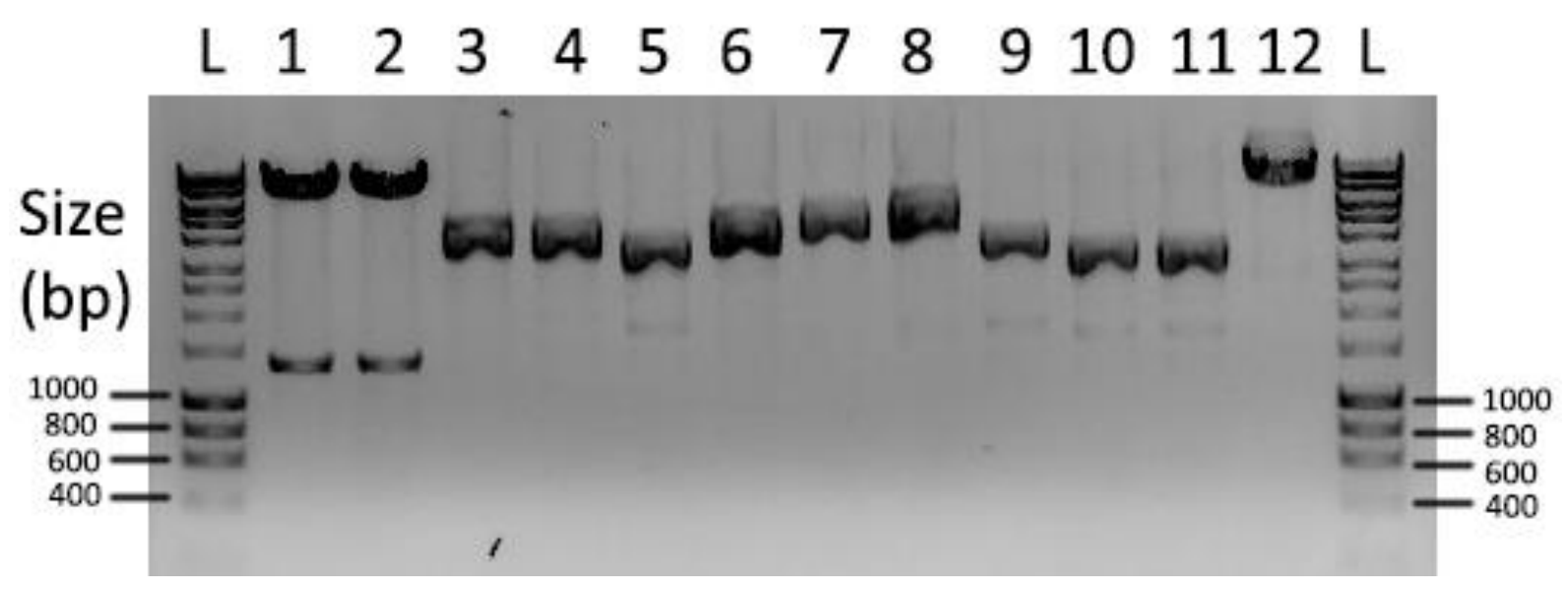

Figure 4-4: BepA2 insert screening in pGBKT7. In this figure, BepA2 inserts within pGBKT7 were screened via double digest, following cloning. Colonies screened in lanes 1 and 2 had inserts slightly larger than the expected size. Lanes 3 - 11 vary in size. Lane 12 showed an empty plasmid. See Appendix Figure 5 for sequencing data of plasmids in lanes 1 and 2.

Bands of the correct size were observed in screened colonies following BepA1 cloning, so those plasmids were sequenced, revealing a successful insertion of bepAl into pGBKT7, in the correct orientation and with the GAL4-BD in frame. Bands slightly larger than the expected size of bepA2 were observed in screening following bepA2 cloning; however, sequencing of 
these plasmids revealed a mutation in the start codon of the bepA2 gene, preventing transcription (see Appendix Figure 6).

The varying sizes of bepA2 inserts observed during screening of bepA2 clones suggested that different recombination or plasmid rearrangement events were occurring (see Figure 4-4). A possible explanation for this observation could be that pGBKT7 was altered by E. coli-driven mechanisms, removing or modifying the bepA2 sequence while retaining the essential kanamycin antibiotic resistance cassette. This process would result in varying plasmid sizes.

As cloning bepA2 into pGBKT7 had proven unsuccessful, cloning into alternative vectors was attempted under the theory that unsuccessful cloning was due to the nature of the vector. A topoisomerase cloning kit was implemented attempting to clone bepA2 into the plasmid pCR®2.1-TOPO®; however, this produced no viable clones. If expression of BepA2 was negatively impacting the Top10 E. coli cloning strain, resulting in recombination events and a modified bepA2 gene, we speculated a low copy number plasmid could reduce the disturbance of bepA2 cloning on the cloning strain. The low copy number plasmid pGBT9, supplied in the Yeast Two-Hybrid System kit (Takara Bio) was selected for bepA2 cloning. pGBT9 has a copy number of 15-20 in E. coli, under the lower copy Col E1 origin of replication, compared to pGBKT7 which has a copy number of $\sim 500$ in E. coli, under the pUC origin. However, this also produced no viable clones.

Upon closer inspection of the primers used to amplify bepA2 from the B. quintana JK31 genome, we discovered the BepA2_R primer did not contain a complete BamHI restriction site (see Table 2: Primers Used in Cloning). Therefore, the digestion using NdeI and BamHI during cloning only digested one end of the BepA2 insert and ligation to the digested pGBKT7 plasmid would only ligate one end. The resulting plasmid and insert would be a linear piece of DNA and may have undergone recombination to circularise. This faulty primer could not be corrected within the time available. As cloning of bepA2 was unsuccessful, only BepA1 was used for the following experiments.

\subsection{Yeast Two-Hybrid Assay}

Prior to carrying out the yeast two-hybrid screen, a series of control experiments were carried out to ensure the protocols were working. As a positive control experiment, the supplied plasmids pGBKT7-53 (encoding GAL4-BD fused with murine p53) and pGBKT7-T (encoding GAL4-AD fused with SV40 large T-antigen) were transformed into yeast. As p53 and large T- 
antigen are known to interact in a yeast two-hybrid assay, pGBKT7-53 in Y2HGold yeast and pGBKT7-T in Y187 yeast were mated, producing diploid yeast containing both plasmids and with all four reporters activated. This showed a positive interaction, as yeast survived on media deficient in leucine and tryptophan and containing the antibiotic aureobasidin A. In addition, yeast colonies turned blue in the presence of $\mathrm{X}-\alpha-\mathrm{Gal}$, indicating full expression of reporters. A negative control was also included by mating pGBKT7-Lam, expressing GAL4-BD with lamin, a protein known not to interact with the large T-antigen, expressed by pGBKT7-T. As expected, the diploid yeast did not survive on deficient media containing aureobasidin A and no colonies were recovered. To demonstrate BepA1 did not auto-activate the reporter promoters, BepA1 was expressed in the absence of any interacting protein; no reporter activation occurred, and the yeast did not survive on deficient media containing aureobasidin A (see Appendix Figure 7). To demonstrate that expression of BepA1 was non-toxic in haploid Y2HGold Yeast, the colony sizes of yeast transformed with BepA1 were compared to those carrying an empty plasmid (see Appendix Figure 8). If BepA1 was toxic, a notable decrease in colony size would be expected compared to an empty plasmid; this was not observed.

pGBKT7-BepA1 was transformed into Y2HGold haploid strain with a transformation efficiency of $7.4 \times 10^{4} \mathrm{cfu} / \mu \mathrm{g}$ DNA, then mated with the Y187 haploid strain containing the Mate $\&$ Plate normalised universal human cDNA library (Takara Bio), resulting in a library of diploid yeast containing the bait protein and the library plasmid PT3718-5 with prey proteins. The mated, diploid culture was plated onto double drop out (DDO) plates, deficient in both tryptophan and leucine, selecting for yeast with both the bait and plate plasmids but without assaying for reporter activation. This was used to determine the number of clones assayed. For the yeast two-hybrid system to be effective at least $1 \times 10^{6}$ clones must be assayed. In this case, $4.13 \times 10^{6}$ colonies were assayed.

Most diploid yeast will not contain interacting bait and prey proteins; therefore, reporters will not be expressed. Initial selection for diploid yeast expressing two interacting proteins tests for survival on double drop out media containing X- $\alpha-G a l$ and aurobasidin A (DDO-XA). DDOXA media is deficient in both tryptophan and leucine; synthesise of these amino acids is reliant on the plasmids, pGBKT7 and PT3718-5. Survival of yeast on this media ensures the diploid yeast contains the two plasmids. Reporter activation results in aureobasidin A resistance and turns colonies blue in the presence of X- $\alpha$-Gal. Diploid yeast with non-interacting bait and prey proteins will not survive on this media. Reporter activation also allows yeast to synthesize histidine and alanine; for more stringent screening and selection, yeast can be plated on 
quadruple-drop-out media, deficient in tryptophan, leucine, histidine, and alanine, supplemented with X- $\alpha-\mathrm{Gal}$ and aureobasidin A (QDO-XA).

The mated culture was first plated on DDO-XA media to assay for diploid yeast strains expressing proteins that interact with BepA1. Six colonies grew; however, all six were smaller than expected ( $\sim 2 \mathrm{~mm}$, compared to the expected 3-5 mm). The colonies were also white, not blue, indicating not all reporter genes were expressed, as colonies were not blue in the presence of $\mathrm{X}-\alpha-\mathrm{Gal}$. The yeast colonies grew on media containing aureobasidin- $\mathrm{A}$, suggesting there was interaction between bait and prey proteins, resulting in activation of the AUR1-C resistance gene but perhaps the interactions were transient or low affinity and did not trigger activation of the complete reporter array. Screening these colonies on more stringent QDO-XA plates was waived as colonies were only weakly growing on DDO-XA media.

To identify genes encoding candidate prey proteins, plasmids were extracted from the six yeast colonies and transformed into E. coli for amplification, using ampicillin as the selective agent. Ampicillin resistance is conferred by the prey plasmid, PT3718-5, but not the bait plasmid, removing the selection pressure to retain the bait plasmid. In the absence of a selection pressure, the bait plasmid is readily lost. The six prey plasmids were purified and sequenced. Four plasmids encoded a HAS2 antisense RNA 1 sequence (see Appendix Figure 9-11). The remaining two encoded the Myozap protein (see Appendix Figure 13-13). There were four independent hits to HAS2 antisense RNA 1, so further investigation is warranted; however, it wasn't immediately evident what protein might be encoded by a gene annotated as an antisense RNA. For this project, we opted to focus on the potential novel interaction between BepA1 and Myozap. Myozap plays a role in a signalling pathway that connects cell junctions to cytoskeletal rearrangements via interactions with Rho-GTPase family proteins ${ }^{115}$.

To validate the interaction between BepA1 and Myozap, we first developed a strategy to ectopically express BepA1, through transient transfection, in mammalian cells. Myozap has been described in many tissue types, including epithelial cells, but we know little about its expression in specific cell lines. We opted to express BepA1 in HeLa cells, which are epitheliallike cells, and to use these transfected cells to carry out an immunoprecipitation reaction, followed by immunoblotting. Myozap is expressed in HeLa cells; If BepA1 is also expressed and the two proteins interact in HeLa cells, BepA1 would also be enriched during the precipitation of Myozap and could be detected in a subsequent immunoblot. 
First BepA1 was cloned into the mammalian expression vector pcDNA3.1 with a translational fusion of a Myc tag, continuous with the BepA1 sequence. The mammalian expression vector allows BepA1 to be transfected and expressed at high levels, from a cytomegalovirus promoter, within HeLa cells. The Myc tag allows BepA1 to be targeted by an anti-Myc antibody by immunoblot.

BepA1 was amplified from the $B$. quintana JK-31 genome by PCR with engineered NheI and BamHI restriction sites (see Table 2: Primers Used in Cloning). Additionally, as expression will occur within eukaryotic cells, a Kozak ribosomal binding sequence was included to allow translation of the transcribed mRNA. The product size was verified by gel electrophoresis then bepAl was cloned into the unique NheI and BamHI restriction sites in the multiple cloning locus of pcDNA3.1. The presence of an insert was then checked by double digest and gel electrophoresis. Plasmids isolated from four colonies were sequenced to confirm there were no errors introduced during PCR. The pcDNA3.1 with bepAl plasmid was transiently transfected into HeLa cells. The cells were incubated for 48 hours to ensure expression of transfected BepA1 had time to occur. A positive transfection control was included, where a pcDNA3.1GFP-yopJ-catmut plasmid, containing a catalytic mutant of B. quintana yopJ, was transfected into HeLa cells. Expression of GFP from this plasmid enabled the visualisation of transfection efficiency (more than $50 \%$ of cells were fluorescent, expressing the inserted gene). Although the transfection efficiency of HeLa cells transfected with pcDNA3.1-BepA1 could not be directly assessed, it was expected to be comparable to the transfection efficiency of pcDNA3.1GFP-yopJ-catmut. Cells were then collected, pelleted, and resuspended in lysis buffer for coimmunoprecipitation. As controls, HeLa cells, either transfected with an empty pcDNA3.1 plasmid or untransfected, were lysed and immunoprecipitated using a goat anti-Myozap antibody and magnetic Protein A beads. The immunoprecipitated solution containing Myozap and any associated proteins was boiled to denature, separating interacting proteins. These protein solutions were then separated by SDS-PAGE and transferred onto a nitrocellulose membrane. The membrane was blotted with the primary mouse anti-Myc antibody, then screened with a secondary HRP-conjugated anti-mouse antibody. The blot was treated with Enhanced Chemiluminescence (ECL) colour reagent, producing visual bands when imaged. 


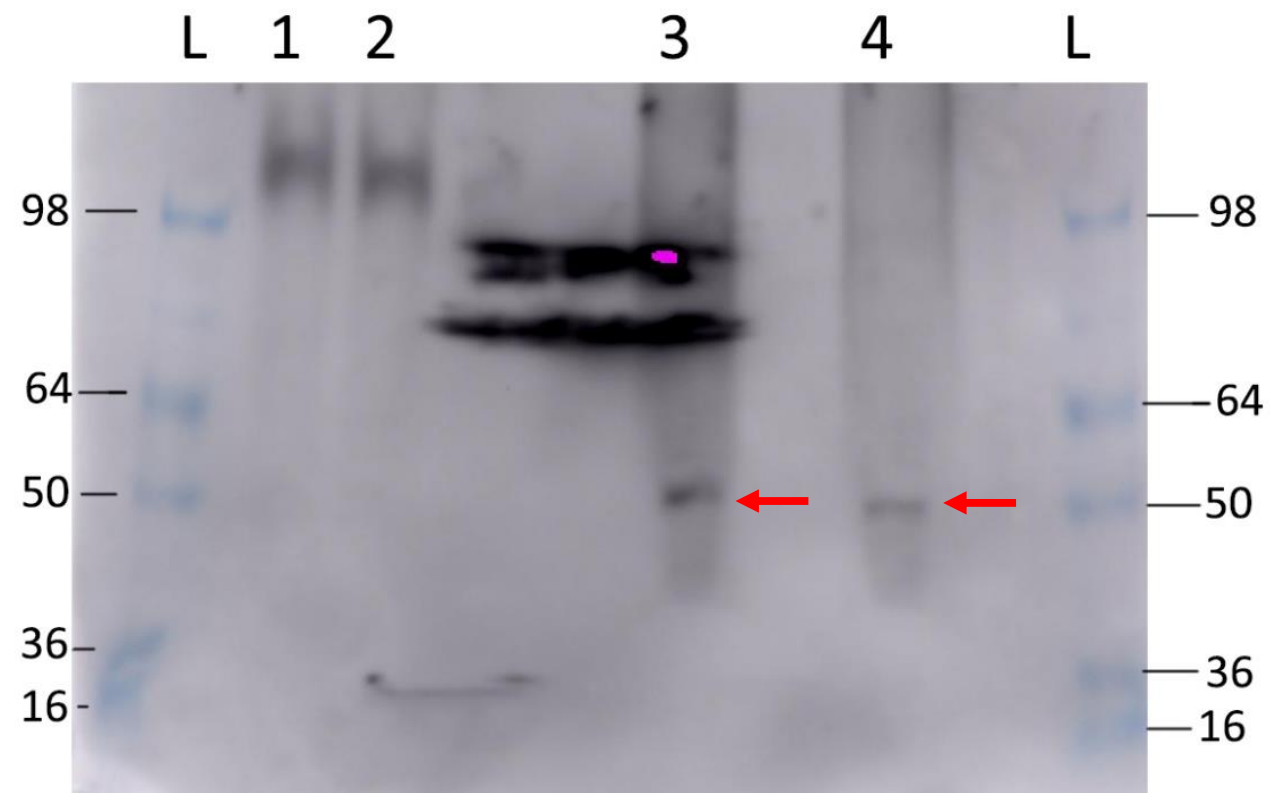

Figure 4-5: Immunoblot of anti-Myozap precipitated proteins; BepAl was not detected in the HeLa lysate of transfected cells. L - SeeBlue Plus2 ladder, 1 - Co-IP: HeLa lysate, transfected with BepA1, 2 - Co-IP: HeLa lysate, untransfected, 3 - HeLa lysate, BepA1 transfected, 4 - HeLa lysate, untransfected. Sizes are in kDa. The Western blot detected the endogenous Myc protein of 50-kDa in lanes 3 and 4 (HeLa cell lysates, indicated with red arrow), but did not detect the expected 26-kDa Myctagged BepA1 in lane 3 (HeLa cell lysate transfected with BepA1), nor did BepA1 appear as a band in lane 1 (co-immunoprecipitation).

The positive control in lane 3 was expected to contain BepA1, appearing as a band at $25 \mathrm{kDa}$, as the primary antibody, anti-myc tag, should bind to transfected BepA1, which should be present in the lysate. Upon further investigation it was noted that the BepA1 myc tag was missing, preventing any binding of the primary antibody and subsequent detection. Therefore, the presence or absence of BepA1 in lanes 1 or 3 could not be determined. A repeat of these experiments would help validate the interaction between BepA1 and Myozap; however, due to time constraints these experiments could not be conducted. 


\subsection{Discussion}

Following the results of chapter 3, technical hurdles and contamination issues made carrying out further experiments using live B. quintana infeasible. Therefore, we turned to alternative experimental techniques to further our investigation into $B$. quintana-host cell interactions. We observed ineffective internalisation of $B$. quintana by THP-1 macrophages and proposed that B. quintana inhibits phagocytosis. As bacteria frequently secrete effector proteins to modulate host cell processes, we hypothesised that BepA1/2 could be responsible for inhibition of phagocytosis in THP-1 cells, possibly via AMPylation of actin cytoskeletal components. Additionally, BepA1 contains a FIC domain, which has been shown for some bacterial pathogens to modulate Rho-GTPase function through AMPylation of the switch region, inhibiting interaction with downstream signalling partners. FIC domains are an enzymatic, conserved motif appearing in the human protein HYPE and bacterial pathogens ${ }^{96}$. FIC domain activity catalyses a reversible AMPylation event where an AMP moiety is added to a conserved tyrosine residue ${ }^{96}$. Some characterised FIC domains have been shown to target Rho-GTPases, inactivating them. As Rho proteins are essential, their inactivation would be expected to have a cytotoxic effect; though, some bacteria have adopted FIC domain-containing proteins to modulate host cell signalling. To give an example of a FIC domain-containing effector protein deployed by bacterial a pathogen, Vibrio parahemeolyticus deploys VopS, which AMPylates Rho-GTPases, blocking actin assembly in host cells; although in this case this results in cell rounding and death ${ }^{116}$. Most bacterial toxins containing FIC domains are cytotoxins, killing host cells by disrupting the essential Rho-GTPase signalling proteins. The non-FIC domaincontaining C3 toxin secreted by Clostridia targets Rho-GTPases disturbing macrophage functions such as phagocytosis and migration ${ }^{117}$. It's possible BepA1/2 is secreted by the Type IV secretion system of B. quintana following phagocytosis by THP-1 cells and inhibits further phagocytosis. This chapter aimed to identify protein-protein interactions between BepA1/2 and host cell proteins to determine if cell signalling is being altered. The targets of the BepA1 enzymatic FIC domain was unknown; by identifying these interactions the activity BepA1 can be better characterised and understood.

\section{Yeast Two-hybrid Assay}

The Bartonella effector proteins released by the T4SS are essential for Bartonella to establish infection, as bacteria without functional T4SS are unable to establish intra-erythrocytic infection, and bacteraemia does not occur ${ }^{118}$. Gaining a clearer understanding of the mechanisms behind the activity of the secreted Beps may help us understand how B. quintana causes disease and interacts with the host. Ultimately this improved understanding will facilitate 
Chapter 4: BepA1 Protein Interaction Studies

development of improved diagnostic screening techniques, disease interventions, and treatments. BepA2 could not be cloned in E. coli, so only BepA1 was screened for proteinprotein interactions. To identify protein-protein interactions a yeast two-hybrid screen (Y2HS) was used. The Y2HS has been used numerous times in literature to identify targets of bacterial effector proteins. For example, Kim et al. successfully used the Y2HS to identify the target of the Pseudomonas syringae effector protein ArvPtoB ${ }^{119}$. In another example, the Y2HS was successfully used to identify the host cell target of the Salmonella enterica effector protein SlrP $\mathrm{P}^{120}$.

The Y2HS challenges the bait protein (BepA1) against a library of host cell proteins, referred to as the prey. This assay resulted in growth of six independent colonies, all of which grew slowly and did not turn blue. This indicated partial expression of the reporter array and may indicate a weak or transient interaction between bait and prey proteins. As survival of the colonies suggested a degree of interaction, the prey plasmids were recovered, sequenced, and a BLAST search was used to identify the interacting library proteins. We identified two independent hits against a protein known as Myocardium-enriched zonula occludens-1associated protein (Myozap), suggesting a possible interaction with BepA1. Due to the mild reporter array expression, yeast from Myozap hits did not survive more stringent screening procedures. There may only be a transient interaction between BepA1 and Myozap, which may not promote total expression of the reporter array, allowing survival. However, since BepA1 is known to act as an enzyme, Myozap could be covalently modified by BepA1 AMPylation; this possibility remains to be tested in the future. The Y2HS is an effective starting point in the identification of effector protein targets, and these hits will be validated independently and further characterised. Validation of the interaction between BepA1 and Myozap became our next goal; to achieve this a co-immunoprecipitation was performed to isolate BepA1 from purified Myozap.

\section{Myocardium-enriched zonula occludens-1-associated protein}

Myozap, first characterised in 2010 as a $54 \mathrm{kDa}$ cardiac protein, is a component of intercalated disks, directly binding junction proteins zonula occludens-1 (ZO-1) and desmoplakin. Myozap is highly expressed in cardiac and lung tissue but was later discovered to be expressed in epithelial and endothelial cells. Myozap provides a link between the intercalated disc and cytoskeletal signalling, binding myosin phosphatase-RhoA interacting protein (MRIP), which binds actin and negatively regulates Rho activity ${ }^{121}$. MRIP binds myosin phosphatase via its CC2 domain while separately binding RhoA via its adjacent coiled domain, complexing myosin 
phosphatase and RhoA together, facilitating the inhibitory regulation of RhoA ${ }^{122}{ }^{121}$. Myozap strongly activates serum response factor (SRF)-dependent transcription in a Rho-dependent manner ${ }^{115}$. Knockdown of the Myozap ortholog in zebrafish resulted in contractile dysfunction and cardiomyopathy demonstrating the involvement of Myozap in the pathogenesis of cardiomyopathy ${ }^{115}$. Myocardin-related transcription factor (MRTF)-A is a transcription factor regulated by actin; actin changes in polymerisation allow MRTF-A to move from the cytosol to the nucleus and carry out its function. In addition, MRTF-A is a co-factor for the SRF pathway, which Myozap also activates. MRTF-A and Myozap and have not been demonstrated to directly interact; however, the signalling network between SRF, actin, and MRTF-A is modified at multiple points by Myozap. Therefore, although BepA1 has not yet been demonstrated to modulate MRTF-A, disturbing the signalling network controlling its activity may affect this transcription factor. MRTF-A promotes NF- $\kappa \mathrm{B}$ activation in response to LPS; silencing of MRTF-A results in inhibition of NF- $\kappa B-d e p e n d e n t$ signalling ${ }^{123}$. LPS stimulates MRTF-A recruitment to pro-inflammatory promoters in an NF- $\kappa B$-dependent manner; Reciprocally, NF- $\mathrm{kB}$ nuclear enrichment is MRTF-A dependent. In addition, MRTF-A

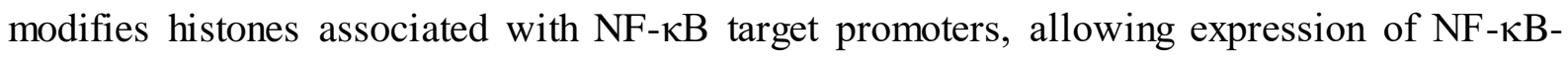
regulated immune genes ${ }^{123}$. NF- $\kappa B$ is active in many cells and a critical signalling pathway in the innate immune response; activation in macrophages stimulates expression of a plethora of genes which drive immune activity, including cytokine secretion ${ }^{124}$. In endothelial cells, NF$\kappa \mathrm{B}$ promotes expression of intercellular adhesion molecule 1 (ICAM-1), a prevalent cell surface receptor that attracts immune cells such as neutrophils ${ }^{125}$. In vascular endothelial cells MRTFA, paired with NF- $\kappa B$, plays a role in the regulation of ICAM-1 expression ${ }^{126}$. Expression of ICAM-1 in vascular endothelial cells allows immune cells to adhere and migrate from capillaries into the tissue during diapedesis to fight infection. The inhibition of ICAM-1 expression in vascular endothelial cells would greatly benefit $B$. quintana if the bacteria are persisting in close association with these cells, as it would allow B. quintana to suppress signalling that would attract neutrophils and other immune cells. As another interesting note, there is a tentative link between Bartonella and Myozap as cardiomyopathy results both from Myozap knockdown, demonstrated in the zebrafish model ${ }^{115}$, and has been reported to occur following Bartonella infection. Such cases, while rare, have been reported in a dog and in several young elite athletes, who died suddenly from cardiomyopathy and whose tiss ues were later found to harbour Bartonella spp. ${ }^{127-129}$. Although speculative, this suggests Bartonella interactions with host Myozap bear further investigation. 
Chapter 4: BepA1 Protein Interaction Studies

\section{Validation of Protein-protein Interactions Between BepA1 and Myozap}

To confirm the interaction between BepA1 and Myozap was genuine, a coimmunoprecipitation and Western blot were deployed to confirm the interaction between the two proteins that was suggested by the yeast two hybrid assay. BepA1 was cloned into a mammalian expression plasmid and transfected into HeLa cells, then a co-immunoprecipitation was used to isolated Myozap from the HeLa cell lysate. Untransfected HeLa cell lysate was used as a control alongside BepA1 transfected HeLa cells. Myozap has not been shown to be expressed in immune cells; it is much more highly expressed in cardiac, lung, endothelial, and epithelial tissue. The immunoprecipitated Myozap protein complex was separated by size, then a Western blot was preformed using a Myc-tag specific antibody to detect any interacting BepA1 protein. The positive control containing whole cell lysates from BepA1-transfected HeLa cells showed no visible band. This indicated that BepA1 protein was not detectable using our experimental method, even in a sample known to contain BepA1. Therefore, there was a technical problem with the experimental method. The fault was established as the BepA1 protein had no attached Myc tag; therefore, the anti-Myc antibody used to detect the protein would not bind, resulting in a negative result, even in the positive control. With extra time, repeating this process with BepA1 and ensuring the Myc tag is functional, would be a valuable step in confirming the validity of the interaction between BepA1 and Myozap. 


\section{Chapter 5: Functional Assays of BepA1 Transfected Cells}

\subsection{Introduction}

Myozap was first characterised by Seeger et al. in 2010 as a $54 \mathrm{kDa}$ cardiac protein highly expressed in heart and lung tissue ${ }^{115}$. In cardiac tissue Myozap is a component of intercalated disks, directly binding zonula occludens-1 and desmoplakin. Intercalated disks are adherence structures between cardiac cells that support synchronised contraction by promoting diffusion of ions between cells via numerous gap junctions ${ }^{130}$. Myozap provides a link between the intercalated disc and cytoskeletal signalling, as it binds myosin phosphatase-RhoA interacting protein (MRIP), which negatively regulates Rho activity. Myozap is therefore a component of the Rho-dependent signalling pathway ${ }^{115}$. Myozap strongly activates SRF-dependent transcription via its Ezrin/radixin/moesin (ERM)-like domain in a Rho-dependent manner ${ }^{115}$. Myozap was subsequently shown to be expressed in a range of epithelial and endothelial cell tissues; for example, Myozap is a component of adherens junctions between vascular endothelial cells ${ }^{131}$.

This chapter aims to detect any cellular changes in HeLa cells transfected with BepA1. If BepA1 interacts with Myozap through AMPylation-driven inhibition, the cellular effects on HeLa cells will reflect inhibition of Myozap. We hypothesised BepA1 was covalently modifying Myozap by AMPylation. As RhoA is involved in cytoskeletal rearrangements and cell migration, a cell motility assay was used to determine if BepA1 altered actin cytoskeleton function in HeLa cells. If Myozap is inhibited by BepA1, cytoskeletal dysregulation may result. We also hypothesised that Myozap inhibition could affect signalling mediated by SRF and NF$\kappa \mathrm{B}$, resulting in changes to HeLa cell pro-inflammatory cytokine secretion. Pro-inflammatory cytokine secretion of BepA1-transfected HeLa cells, compared to untransfected, was assessed by stimulation of cells with the pro-inflammatory cytokine TNF- $\alpha$, followed by quantitation of supernatant IL- 6 and IL-8 by ELISA.

\subsection{Cell migration assay}

To investigate If BepA1 affects the actin cytoskeleton, co-ordinating wound repair, we used a scratch assay to identify differences in cell migration between HeLa cells transfected with BepA1 compared to untransfected HeLa cells or HeLa cells transfected with the empty 
pcDNA3.1 plasmid. RhoA signalling is known to be required for cell migration ${ }^{132}$, and we wanted to test whether BepA1 could be inhibiting RhoA activity via interactions with Myozap.

HeLa cells were grown to a confluent, adherent monolayer. A part of the monolayer was removed by gently scratching the cells with a sterile pipette tip; the cells were gently washed to remove cell debris and photographed at various time intervals to measure the rate of wound area closure (see Figure 5-1). Under these conditions, the cells migrate in to close the gap to reform the monolayer. In the first 24 hours, the gap is primarily filled by cell migration, with proliferation contributing at later time points ${ }^{132}$. The duration required for the wound area to close can be compared between conditions to indicate any dysfunction of RhoA or cytoskeletal signalling.
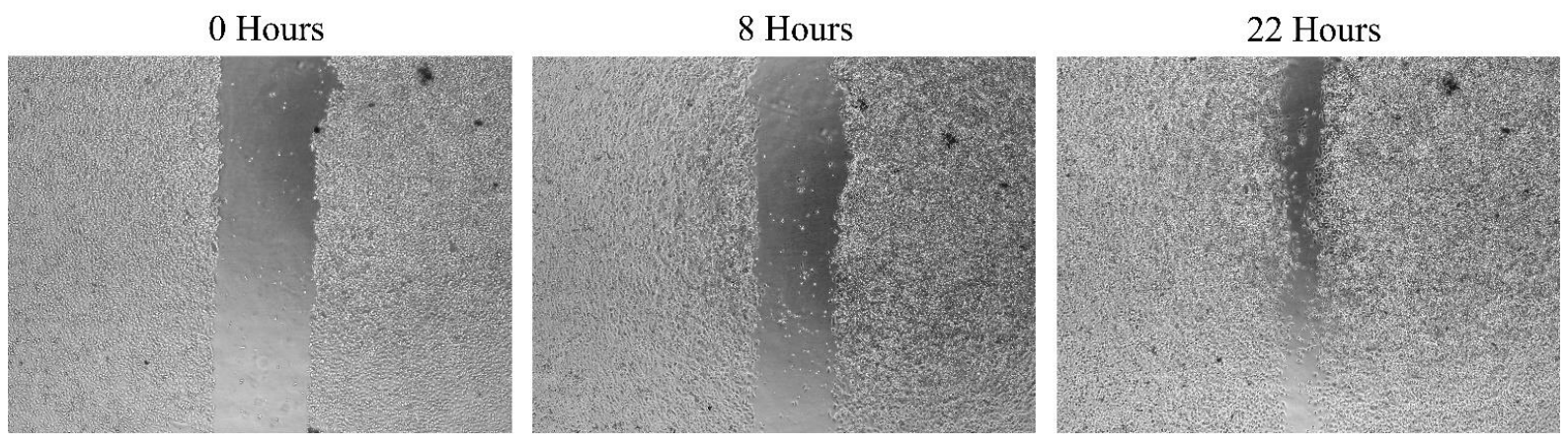

Figure 5-1: Wound Repair in HeLa cells Transfected with BepAl over 22 hours. The wound area (the area absent of cells) decreases over time as HeLa cells migrate inwards, filling the space. Wound area was calculated using the image processing software ImageJ and the area calculating macro MRI Wound Healing Tool. 


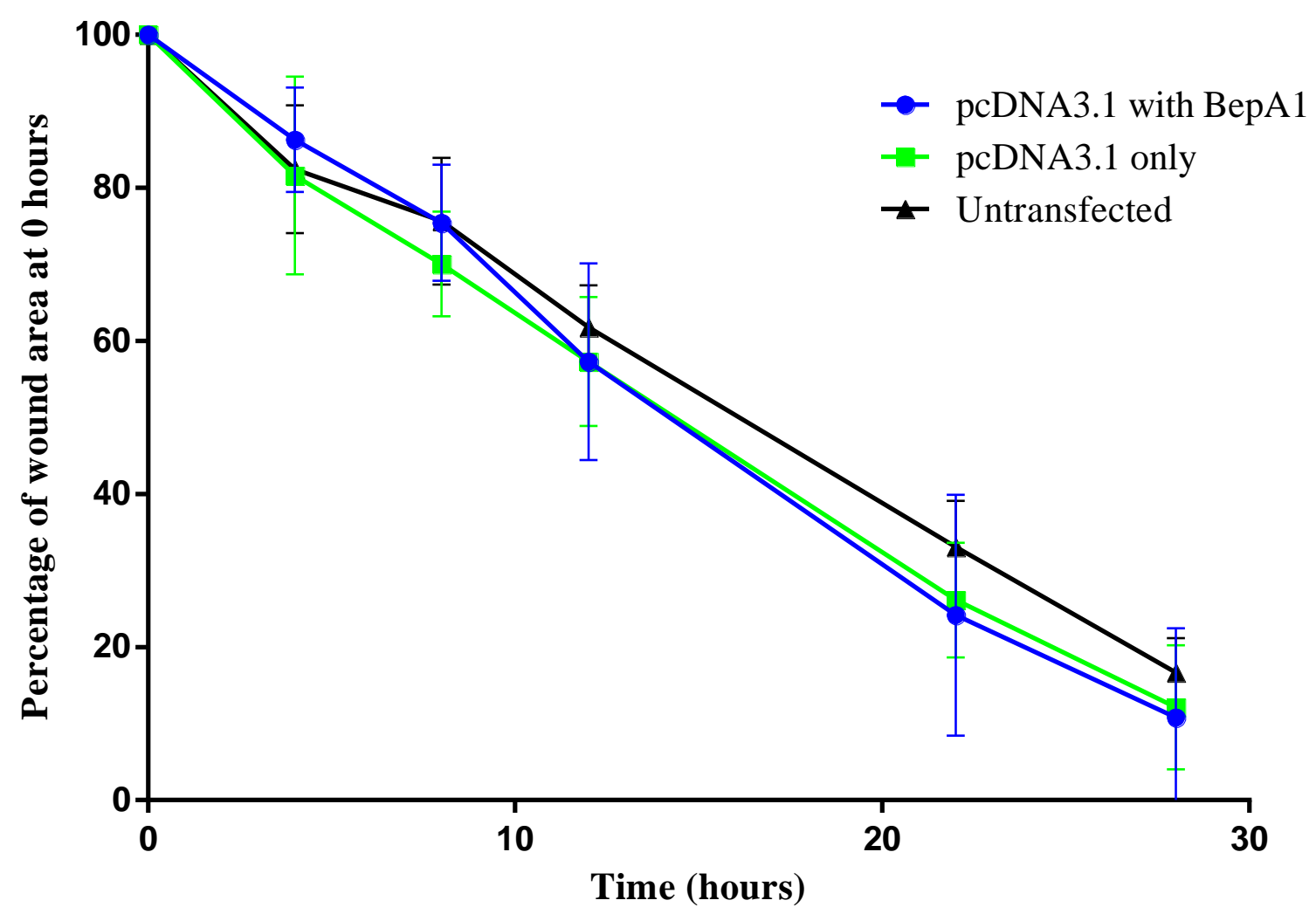

Figure 5-2: Cell migration assay showing no significant difference between BepAl transfected HeLa cells and untransfected. Ten biological replicates were tested for both pcDNA3.1 with BepA1 and pcDNA3.1 only conditions. Four biological replicates were tested for untransfected cells. Transfection efficiency of these cells was estimated at above $50 \%$ based on transfection of HeLa cells with a similar sized plasmid which encoded GFP, allowing assessment of transfection efficiency of the protocol by fluorescent microscopy.

No significant difference in the rate of wound repair was observed between untransfected HeLa cells, HeLa cells transfected with empty pcDNA3.1 plasmid, or HeLa cells transfected with BepA1. This suggests there is no dysregulation of the cytoskeleton occurring in HeLa cells transfected with BepA1 that is pronounced enough to significantly affect migration ability.

This experiment lacked a positive control, presenting the wound repair capabilities of cells incapable of actin rearrangement. Although the inclusion of this control would not have altered the results of this experiment, in future a latrunculin treatment group could be included, where actin polymerisation is inhibited. However, the role of RhoA and actin reorganisation in wound repair has been well established. 


\subsection{Cytokine Assays}

Myozap has been identified as a potential target of BepA1 activity in host cells, and Myozap can affect pro-inflammatory cytokine secretion through SRF and NF- $\kappa \mathrm{B}$ signalling modulation. Therefore, we investigated if BepA1 transfected HeLa cells differed from untransfected HeLa cells in the concentration of secreted IL- 6 and IL- 8 cytokines. Both IL-6 and IL- 8 are expressed as danger signals by host cells in response to pathogens and result in leukocyte recruitment to sites of infection. Inhibition of pro-inflammatory cytokine secretion in BepA1-transfected cells would indicate an immune suppressive function. $B$. quintana deploys numerous immunosuppressive mechanisms to survive within the host and establish acute and chronic infection, identifying the origin of these effects is essential in understanding B. quintana infection.

HeLa cells release very low concentrations of pro-inflammatory cytokines when unstimulated, so to determine if BepA1 inhibits cytokine production, the cells were stimulated to release cytokines, to reveal any inhibition. Transfected HeLa cells were seeded into 24- well plates and stimulated with TNF- $\alpha$, a potent pro-inflammatory signalling molecule that induces proinflammatory cytokine production and secretion through $\mathrm{NF}-\kappa \mathrm{B}$ stimulation. HeLa cells were left enough time to respond to the stimulus by producing and secreting pro-inflammatory cytokines; the following day, IL-6 and IL-8 concentrations in supernatants were quantified by ELISA. 


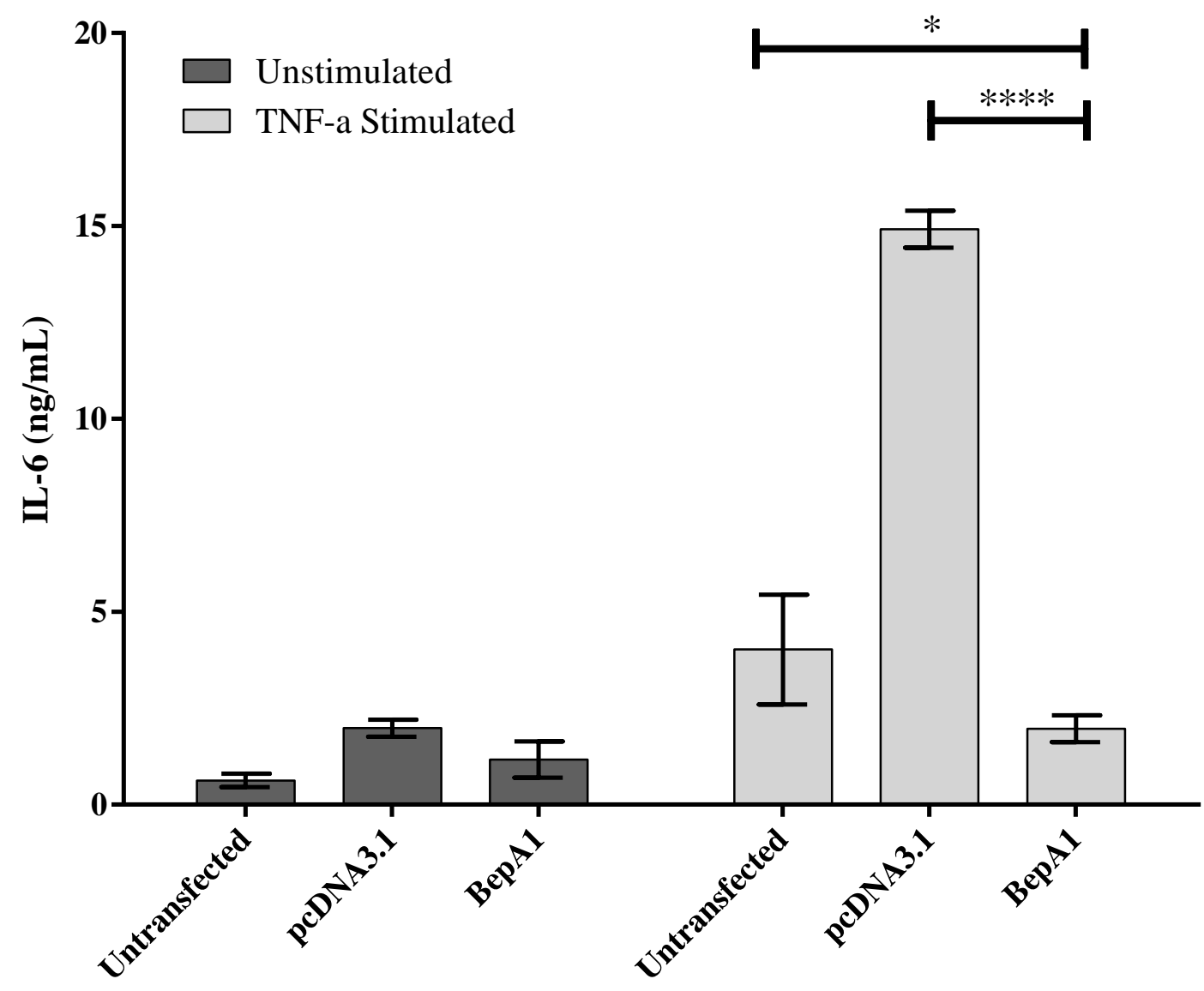

Figure 5-3: IL-6 ELISA displaying a significant difference between BepAl transfected HeLa cells and controls. A statistically significant decrease in IL-6 secretion was observed in BepA1-transfected HeLa cells compared with both controls: untransfected HeLa cells and pcDNA3.1 transfected HeLa cells. An increase in IL-6 secretion was observed in pcDNA3.1 transfected HeLa cells in response to stimulation, which was likely a reaction to the combination of TNF- $\alpha$ and the presence of dsDNA resulting from the mammalian plasmid. *p value $=0.0308$ comparing untransfected group to BepA1 transfected group. $* * * * \mathrm{p}$ value $=<0.0001$ comparing pcDNA3.1 transfected group to BepA1 transfected group. 


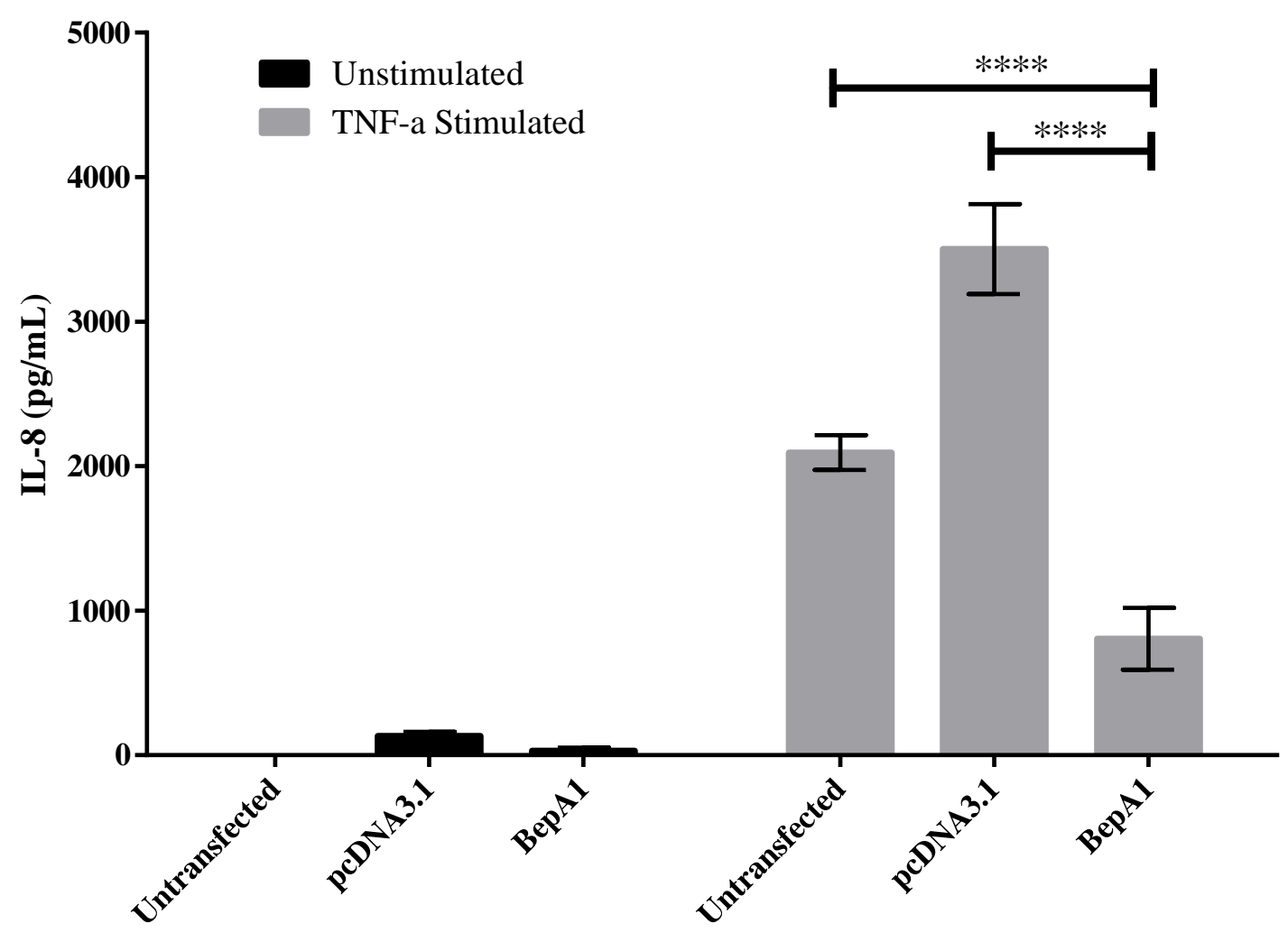

Figure 5-4: IL-8 ELISA displaying a significant difference between BepA-transfected HeLa cells and controls. A statistically significant decrease in IL-8 secretion was observed following TNF- $\alpha$ stimulation in BepA1-transfected HeLa cells compared with both controls, untransfected HeLa cells and pcDNA3.1-transfected HeLa cells. As with IL-6 secretion, an increase in IL-8 secretion was observed in pcDNA3.1 transfected HeLa cells, relative to untransfected HeLa cells, in response to stimulation, likely resulting from the presence of pcDNA3.1, as double-stranded cytoplasmic DNA can activate internal cell immune sensors. $* * * * p$ value $=<0.0001$ comparing untransfected group or pcDNA3.1 transfected group to BepA1 transfected group.

A reduction in pro-inflammatory cytokines IL-6 and IL-8 was observed between BepA1 transfected HeLa cells and pcDNA3.1 transfected and untransfected HeLa cells (see Figure 5-3 and Figure 5-4). These data suggest the expression of BepA1 in HeLa cells does cause NF- $\mathrm{B}$ inhibition, resulting in reduced pro-inflammatory cytokine secretion in response to stimulation. Although the interaction between Myozap and BepA1 has not been verified, a functional consequence of BepA1 activity is identified here, a finding that is compatible with our hypothesis that BepA1 AMPylates Myozap. If the quantification of pro-inflammatory cytokines was to be repeated, an additional control could be included. It would be useful to include HeLa cells transfected with a catalytic mutant BepA1, where the FIC domain is non-functional. This would confirm that the observed NF- $\mathrm{BB}$ inhibition is dependent on the enzymatic activity of BepA1. 
Chapter 5: Functional Assays of BepA1 Transfected Cells

\subsection{Discussion}

Protein interaction studies suggested that Myozap could be a possible target of BepA1; because BepA1 has a Fic motif, we wondered if Myozap could be covalently modified by BepA1, perhaps losing certain functions. Based on what is known about the signalling pathways that Myozap may influence, including RhoA signalling pathways and SRF and NF- $\mathrm{BB}$ transcription factors, we opted to assess cell migration and cytokine secretion from cells transfected with BepA1 and controls. Myozap may control actin dynamics through the binding of MRIP, which influences Rho signalling and subsequently alters actin rearrangements. Actin alterations may in turn modulate transcription factors MRTF-A and NF- $\kappa \mathrm{B}$, which control cytokine expression and secretion.

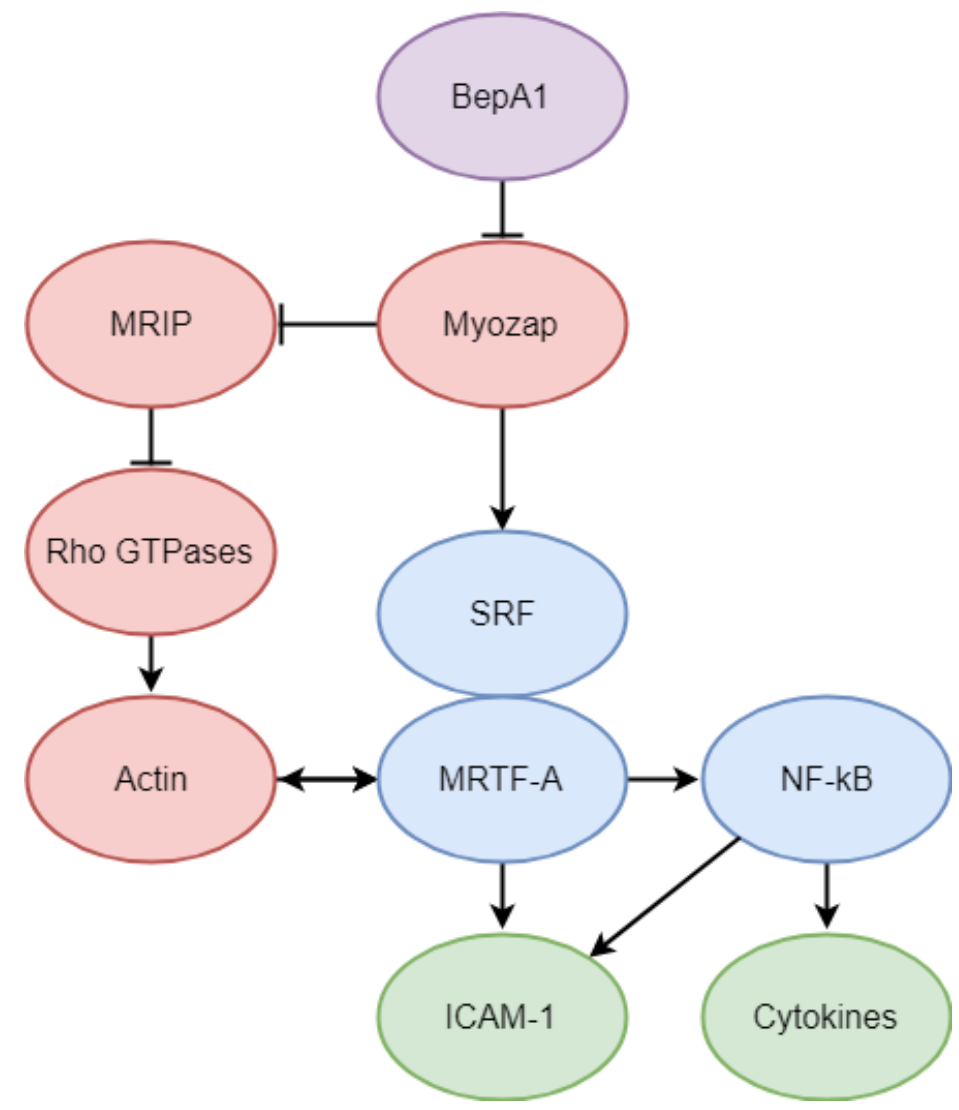

Figure 5-5: Possible signaling pathways affected by BepA1, resulting in inhibition of cytokine secretion. Arrows indicate stimulation of the target proteins while blunt lines indicate inhibition. We hypothesise that the B. quintana effector protein BepA1 (seen in purple) is inhibiting Myozap by AMPylation via the FIC domain. Physiologically, Myozap activity is involved in stimulation of transcription factors NF$\kappa \mathrm{B}$ and MRTF-A (seen in blue). This activity occurs either through interactions with the transcription factor SRF (seen in blue) and the cofactor MRTF-A, or by modulation of MRIP, influencing actin signaling proteins (seen in red). Actin signaling may influence cell motility in addition to regulation of

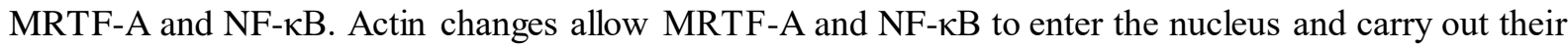
function. Cytokine secretion and ICAM-1 upregulation (seen in green) are functional effects resulting from this signaling pathway. 
As Myozap influences Rho signalling, which has ties to actin cytoskeletal signalling, cell migration was tested using a wound repair assay to determine if BepA1 modulation of Myozap had an effect. NF- $\mathrm{B}$ is central in responding to pathogens, upregulating pro-inflammatory cytokines to recruit leukocytes to the site of infection. Numerous bacterial pathogens have evolved a wide range of mechanisms to interfere with the $\mathrm{NF}-\kappa \mathrm{B}$ pathway at every step to inhibit its effective deployment by the host ${ }^{133}$. As Myozap interacts with SRF, which impacts NF- $\kappa \mathrm{B}$, pro-inflammatory cytokine secretion may be affected. Levels of pro-inflammatory cytokines IL-6 and IL-8, secreted by HeLa cells stimulated with TNF- $\alpha$, were assessed via ELISA.

We hypothesised that proinflammatory cytokines IL-6 and IL-8 would be significantly decreased in BepA1-transfected cells. In addition, cytoskeletal rearrangements required for migration would be impaired, reducing wound repair efficiency in BepA1 transfected cells.

\section{Effects of BepA1 on Migration}

The actin cytoskeleton is a frequent target of many bacterial pathogens ${ }^{134}$. These pathogens either directly inject virulence factors into host cells or secrete toxins into the surrounding environment. Bacterial toxins often target Rho-GTPases, catalysing covalent modifications to block their signalling capabilities, resulting in alterations to endothelial and epithelial cell barrier ${ }^{135}$. However, injected virulence factors commonly target Rho-GTPases, mimicking eukaryotic signalling proteins to subvert their function, resulting in modulation of the actindriven process such as phagocytosis or cell migration ${ }^{135}$. For example, in 2009 McLaughlin et al. used a cell migration assay in the study of Salmonella typhimurium and demonstrated the bacteria inhibit cell migration of macrophages and dendritic cells ${ }^{136}$. They further demonstrated this effect results from the effector protein Ssel, which inhibits host cell migration by affecting the host cell migration regulator IQGAP $1^{136}$. Another study published in 2015 by Brothers et al. determined Serratia marcescens inhibits cell migration of corneal cells in vivo via secreted $\operatorname{LPS}^{137}$.

The actin cytoskeleton is regulated in part by MRIP, a target of Myozap. As Myozap has been identified as a possible target of BepA1, transfected cells containing BepA1 may have impaired actin organisation processes, which affect migration. A wound repair assay was deployed to test this hypothesis. A wound repair assay involves scratching a cell monolayer in a uniform manner, then recording the amount of migration the cells undergo to fill the gap, in a certain period. By comparing the BepA1 transfected cells with untransfected cells any difference can 
Chapter 5: Functional Assays of BepA1 Transfected Cells

be recorded. No significant difference was observed between the two groups suggesting the actin cytoskeleton is not greatly impacted by BepA1. This observation may be due to BepA1 not affecting signalling proteins involved in the regulation of actin polymerisation, influencing cell migration. Alternatively, BepA1 may affect these signalling proteins; however, redundancy systems within the signalling network may prevent significant deviation from normal function. It's also possible that even if Myozap is modified by BepA1, the MRIP-binding domain may not be affected, allowing Myozap to retain some signalling activity (see Figure 5-5).

\section{Effects of BepA1 on Cytokine Release}

External stimuli, such as LPS, stimulate PAMPs on the surface of certain types of cells, which activate the MAPK signalling cascade, resulting in NF- $\mathrm{KB}$ expression and activity. NF- $\mathrm{BB}$ activation in endothelial or epithelial cells results in expression and secretion of proinflammatory cytokines such as IL-6 and IL-8. IL-6 and IL-8 secretion in turn attracts immune cells, particularly neutrophils, to the site of infection. To assess if BepA1 affected the secretion of pro-inflammatory cytokines following stimulation, BepA1 transfected HeLa cells were stimulated with TNF- $\alpha$, a strong pro-inflammatory stimulus. A large increase in cytokine production was observed in simulated HeLa cells transfected with an empty plasmid; this effect was blocked in HeLa cells transfected with BepA1. This points towards BepA1 as a virulence factor of B. quintana, deployed not to directly suppress immune cells but to inhibit immune cell recruitment to a site of infection by inhibiting cytokine secretion by epithelial and/or endothelial cells. Endothelial cells are a likely target for this activity as Bartonella spp. are thought to be closely associated with the vascular endothelium during infection. A common strategy of bacterial pathogens is the inhibition of pro-inflammatory cytokines to inhibit appropriate immune cell recruitment to sites of infection. For example, in 2003 Zhou et al. demonstrated Yersinia pestis deploys the effector protein YopJ against bronchial epithelial cells to inhibit proinflammatory cytokine secretion, blocking the innate immune response ${ }^{73}$. B. quintana may be deploying BepA1 in a similar fashion to block an immune response, inhibiting the ability of endothelial cells to effectively raise the alarm in the vascular endothelium.

We also observed that HeLa cells transfected with an empty pcDNA3.1 plasmid showed a significantly increased response in IL-6 and IL- 8 secretion following TNF- $\alpha$ stimulation compared to untransfected controls. This suggests HeLa cells reacted to the transfection protocol with a pro-inflammatory response. We used the transfection reagent Viafect, which is a cationic lipid transfection method where dsDNA in introduced into cells. It is possible that this process mimics viral infection, particularly those caused by DNA viruses that replicate 
Chapter 5: Functional Assays of BepA1 Transfected Cells

outside of the nucleus, e.g., pox viruses. The cells likely sense the double stranded cytoplasmic DNA via intracellular immune sensors; this signal, combined with TNF- $\alpha$ stimulation, resulted in much higher levels of IL-6 and IL-8, compared to untransfected cells. This may explain why these cells responded with an amplified immune response, increasing pro-inflammatory cytokine production.

The results of these cytokine production experiments provide evidence that BepA1 was successfully transfected into HeLa cells, as there was a functional effect in BepA1-transfected cells compared to cells transfected with an empty plasmid. In addition, pcDNA3.1 mammalian expression plasmid containing BepA1 was transfected alongside pcDNA3.1 with GFP as a positive control and showed good expression levels, at least $50 \%$ of cells expressing. Both plasmids were similar sizes therefore transfection efficiency would not differ significantly between the two, allowing confidence that pcDNA3.1-BepA1 was transfected into HeLa cells and resulted in BepA1 expression. 


\section{Chapter 6 Research Motivations, Key Findings, and Future Directions}

\subsection{Research Motivation}

My primary motivation for this research was to better understand the interactions between $B$. quintana and the human immune system, particularly those occurring during the early stages of infection. While other Bartonella spp. such as B. henselae, have been the focus of some modern research, the pathogenesis mechanisms of $B$. quintana are difficult to study because reservoir host infection is limited to humans and Rhesus macaque (Macaca mulatta) monkeys. Therefore, animal studies are often not possible, or at least very expensive and difficult, in the case of $B$. quintana. We studied B. quintana using in vitro methods, including cell lines and ectopic expression of effector protein function. These methods are complimented with published in vitro studies of related Bartonella spp. with their reservoir hosts. For example, in 2016 Hong et al. used B. tribocorum as a model to better understand Bartonella transit from the dermal tissue, via the lymphatic system, to the bloodstream ${ }^{60}$. This research also deployed a gentamicin protection assay to assess internalisation of $B$. tribocorum by rat peritoneal macrophages. Hong et al. similarly observed low internalisation of B. tribocorum by macrophages; at an MOI-10, a mean of $136 \mathrm{CFU}$ were engulfed over the experimental duration ${ }^{60}$ compared to an average of 42 in our experiment.

Bartonella infections differ significantly between species; research into B. quintana infection in humans is essential to understand its pathology for the development of better diagnostic screening techniques and treatments. B. quintana is understudied and the impact of B. quintana infection in our community, particularly in vulnerable populations, is not fully appreciated. Treatment and diagnosis of $B$. quintana infection is very difficult and must be improved to lessen the substantial burden on those affected and the healthcare system. Discerning the interactions between Bartonella and host cells essential to fully understand the challenges faced in diagnosis and treatment of the infection.

We directed our focus towards the interactions between $B$. quintana and human macrophages to gain a better understanding of $B$. quintana dissemination from the dermal inoculation site. This research provides insight into the early stages of $B$. quintana infection by characterising previously unexplored interactions between this pathogen and the human immune system. Our 
results revealed that human macrophages take up B. quintana poorly; however, technical hurdles made further research using live B. quintana impossible in the given time frame. Our focus then turned to investigation of a specific Bartonella effector protein, BepA1, as a possible culprit for the apparent lack of phagocytosis by macrophages. We identified a potential target of BepA1, Myozap, a cell signalling protein expressed in cardiac, endothelial, and epithelial tissues, but not immune cells. This indicated BepA1 was likely not the cause of macrophage modulation, but the impact of Myozap targeting was studied further to better understand the role of BepA1 during infection. Our data revealed BepA1 activity in endothelial cells resulted in an inhibitory effect of pro-inflammatory cytokine secretion. This suggests BepA1 is deployed as an indirect immune suppressive virulence factor. Further research could help determine the full implication of BepA1 activity during infection.

\subsection{Key Findings}

Although Bartonella spp. have been studied for decades, research into B. quintana dropped drastically after the 1960s as trench fever was no longer seen as a priority. Some researchers did continue the study of B. quintana past the 1960s; J Vinson worked to fulfil Koch's postulates by culturing the bacteria in a laboratory setting and infecting volunteers ${ }^{138,139}$. $B$. quintana has re-emerged as a dangerous pathogen infecting socially disadvantaged populations causing the disease urban trench fever, as well as other clinical syndromes. B. quintana infections remain difficult to diagnose for multiple reasons, including the effective immune evasion mechanisms of the bacteria and a poor understanding of where in the body the bacteria persist during chronic infection. Treatments of B. quintana infection are not particularly sophisticated, relying on combination antibacterial therapy. This thesis aimed to provide insights into how B. quintana interacts with the innate immune system during early infection. This knowledge builds upon previous studies of other Bartonella spp. and extends our collective understanding of this human pathogen. It is our hope that the key findings of this thesis can influence diagnosis and treatment of B. quintana infection, either directly or by assisting further research.

A large hurdle in the study of B. quintana is the technical considerations of growing the bacteria under laboratory conditions. Bartonella are fastidious, slow growing bacteria, and this is particularly the case with $B$. quintana, which is extremely slow growing and sensitive to environmental perturbation and contamination. Research into B. quintana activity requires the culture of the live bacteria for experimentation, as with the macrophage interaction experiments of this thesis. During this research we determined chocolate agar solid media should be prepared 
as fresh as possible for $B$. quintana growth and should not be used beyond seven days. $B$. quintana must be grown in candle jars, preferably with multiple plates to increase humidity levels in the jars. Finally, B. quintana stocks should be regularly checked for contamination through colony PCR amplification of $16 \mathrm{~S}$ sequence for phylogenetic analysis. Cultivation of $B$. quintana requires lengthy culture on rich media and is very susceptible to contamination issues. It is likely that the difficulties associated with $B$. quintana culture hamper research efforts for this species; hopefully the culture condition optimisation required for this thesis can reduce the burden for subsequent research.

Preliminary macrophage interaction experiments revealed that B. quintana largely resists phagocytosis by human macrophages. As we hypothesised B. quintana would persist intracellularly within macrophages, this was an unexpected result. This suggests B. quintana evades macrophages when contacted in the skin, allowing the bacteria to invade deeper tissues in the host and establish infection. Specifically, evasion of phagocytes would be particularly advantageous for the bacteria, which may encounter large numbers of macrophages during their transit through the lymphatic system. Due to the technical hurdles of culturing B. quintana combined with contamination issues within the lab, further experimentation with live $B$. quintana was not possible. Therefore, we decided to focus on a specific Bartonella effector protein, BepA1. BepA1 contains a FIC domain, which target Rho-GTPases ${ }^{92,96}$, host cell signalling proteins with ties to the actin cytoskeleton. We hypothesised the effect of BepA1 may be causing inhibited phagocytosis in THP-1 cells.

A yeast two-hybrid system assay was deployed to identify specific protein-protein interactions between BepA1 and host cell proteins. We identified the host cell protein Myozap as a potential target of BepA1. BepA1 contains a FIC domain, an enzymatic domain that catalyses AMPylation of target residues. We hypothesise that BepA1 may AMPylate Myozap, modulating its signalling ability. Further research could help determine if this is the case. Myozap expression has been demonstrated in cardiac, epithelial, and endothelial tissues, but not immune cells. Our data revealed a target of BepA1 that is not expressed in immune cells, but instead is expressed in other host cells, such as endothelial cells. B. quintana is a bloodborne pathogen that infects the host, seeding the bloodstream; literature often describes Bartonella spp. interacting with endothelial cells ${ }^{140,141}$. BepA1 may inhibit pro-inflammatory cytokine signalling by endothelial cells to prevent an appropriate response by the immune system, allowing the bacteria to persist in association with these endothelial cells. This activity 
Chapter 6 Research Motivations, Key Findings, and Future Directions

may be a contributing factor in the development of chronic infection, enabling $B$. quintana to survive in the host long periods and to persist as a successful stealth pathogen.

\subsection{Future Directions}

\section{B. quintana Interactions with Other Innate Immune Cells}

Future research into the interactions between B. quintana and immune cells could be expanded to investigate interactions with other cell types. Host-pathogen interaction studies are not wellestablished for the study B. quintana. Little is known about the interactions between $B$. quintana and human immune cells. The first step in understanding how Bartonella behaves during early infection is to investigate these interactions with human cells. The gentamicin protection assay protocol used for these studies could be adapted to a range of other host cell types. We would have extended our research to include many other cells types; however, due to technical hurdles and time constraints this option was not available.

Neurological symptoms have been reported in patients with Bartonellosis ${ }^{142,143}$; a future experiment could investigate microglia cells physiologically resident in the brain, exploring their interactions with $B$. quintana and B. henselae. Microglia have been suggested as a possible reservoir for Bartonella during chronic infection, making them an interesting target for investigation $^{144}$.

Further research into the interactions between Bartonella and dendritic cells would also be valuable to better understand bacteria-host cell interactions within the dermis of the skin. Research into BepE has highlighted dendritic cells as an essential player for B. tribocorum dissemination from dermal tissues into the bloodstream ${ }^{57}$; perhaps further investigation could identify the causative mechanism behind this activity. The mechanisms behind B. quintana dissemination throughout the body are poorly understood; it's possible the bacteria are internalised by dendritic cells and the migration of dendritic cells aids bacterial dissemination. Investigation into internalisation and intracellular persistence of B. quintana within human dendritic cells could help answer these questions.

Neutrophils would also be an interesting target for further investigation. Neutrophils are the most abundant immune cell in the bloodstream, which is the natural habitat of B. quintana. Our results identified a mechanism of BepA1-driven inhibition of pro-inflammatory cytokines, IL6 and IL-8, which are secreted by endothelial and other cells as an effective distress signal during infection. In large part these cytokines recruit numerous neutrophils to a site of infection; 
Chapter 6 Research Motivations, Key Findings, and Future Directions

neutrophils are typically the first responders that flood the infected area in high numbers. It would be interesting to see how effective neutrophils are in the clearance of $B$. quintana, though the degree to which neutrophils encounter $B$. quintana during infection is still uncertain. The ability of neutrophils to internalise and kill B. quintana, through both intra- and extracellular mechanisms, has not been assessed and is a rich area of future research. Neutrophil-mediated killing of B. quintana could be assessed in vitro, and the ability of B. quintana to persist within neutrophils could be determined. Although neutrophils are short lived and are less mobile after encountering pathogens, compared with dendritic cells, pathogens as diverse as Ehrlichia, Anaplasma and Neisseria gonorrheae replicate inside neutrophils, delaying neutrophil apoptosis and extending their lifespan. Further research into the interactions between $B$. quintana and innate immune cells will aid in the understanding of the early stages of infection but also identify numerous questions for further investigation.

\section{B. quintana null mutation Experiments}

Site-directed null mutant bacterial strains are routinely used to assess function of a protein of interest; removing or inactivating the selected protein helps to identify the differences in activity compared to the wild-type strain, granting insight into the functionality of the protein. For example, in 2011 Truttmann et al. used a BepA-G null mutant B. henselae strain to study invasome formation in HUVEC and HeLa cells ${ }^{105}$. A similar approach could be used in the study of BepA1 in B. quintana. A T4SS mutant B. quintana strain that lacks a functional secretion apparatus could be developed to identify B. quintana effects on host cells that are due to the secreted Beps; in the absence of the T4SS, Beps could not be injected into host cells. A BepA1 mutant strain could be used for numerous experiments in the investigation of BepA1 dependent cellular modulation. Finally, a BepA2 mutant strain could be used to determine the degree to which BepA1 and BepA2 interact or complex together to carry out function.

An example of an experiment utilising these mutant strains, the wild-type, T4SS mutant, and BepA2 mutant strains could be used to infect host cells, with BepA1 appropriately tagged to identify translocation. BepA1 does not contain a BID domain; therefore, it potentially lacks the required sequence for recognition by VirB4 and subsequent secretion directly into host cells. BepA1 may complex with BepA2 to pass through the T4SS, pass through without complexing with BepA2, or enter host cells through another mechanism. A suitable tagging option would be a Myc tag for anti-myc antibody targeting; this would allow for western blot screening of host cells for the presence of BepA1. An attached GFP tag could also be used to assess transfer of the effector protein by microscopy or florescence cytometry. There would likely be low 
abundance of BepA1 within host cells, demanding a sensitive microscopy option for detection, such as a confocal microscopy. One consideration of these approaches is false positives from any $B$. quintana remaining in or around the cells, which would contain BepA1. Numerous washes and a gentamicin treatment would kill extracellular B. quintana, but confident clearance of all intracellular bacteria would be more difficult. My results show internalisation of $B$. quintana by THP-1 cells is limited, but nevertheless background signal from the bacteria should be considered. Another option would be FRET-based detection, where BepA1 is expressed as a fusion with TEM-1 beta-lactamase which is cleaved upon entry into host cells freeing the fluorescent FRET dye ${ }^{145}$. This approach has been used in the study of Yersinia effector proteins in vivo, to identify host cells that are injected by the Type III secretion system ${ }^{145}$. If BepA1 can be translocated in the absence of BepA2 and the T4SS, there may be an alternative mechanism at work. The likely result of this experiment would be BepA1 cannot enter host cells in the absence of the T4SS or BepA2, which would strongly suggest BepA1 and BepA2 complex together to allow BepA1 to pass through the T4SS. If BepA1 translocate in the BepA2 mutant strain, it would suggest BepA1 can pass through the T4SS even lacking a BID domain.

\section{Phagocytosis Assay}

The data generated during this project demonstrated B. quintana is not effectively internalised by THP-1 cells; the role of the Bartonella effector protein BepA1 was investigated as a possible cause. It was discovered that BepA1 likely interacts with Myozap, which influences the host actin cytoskeleton. Myozap is not known to be expressed in immune cells. Therefore, our data did not support the hypothesis that BepA1 was responsible for modulation of THP-1 phagocytic activity, although other Beps may be involved. A T4SS mutant strain could be used to identify if other Beps effect phagocytic activity of human immune cells; infecting host cells with a T4SS mutant or the wild type parent strain and comparing numbers of bacteria internalised would reveal differences in levels of phagocytosis. This experiment could be paired with a phagocytosis assay, where pHrodo particles are utilised as a measure for phagocytosis and lysosomal fusion in cells. pHrodo particles are small E. coli fragments with an attached fluorescent bead that fluoresce under low $\mathrm{pH}$ conditions, measured using flow cytometry. As the particles only fully fluoresce when the lysosome fuses with the phagosome, background from extracellular pHrodo particles is minimal. pHrodo particles would be added to THP-1 cells with or without pre-exposure to $B$. quintana. If the phagocytic activity of THP-1 cells is inhibited by B. quintana, the cells will internalise fewer fluorescent particles, giving off a weaker signal. A negative latrunculin control should be included, which almost entirely inhibits phagocytosis. In addition, a heat-treated B. quintana control could be included, distinguishing 
Chapter 6 Research Motivations, Key Findings, and Future Directions

between actin modulation of phagocytic activity vs a passive effect resulting from macrophage contact with $B$. quintana material. Although this experiment would not identify the specific Beps responsible, if any, it would be a starting point for future research by identifying these effector proteins as the root of this immune suppressive mechanism.

\section{Conditioned Media Experiment}

To determine if BepA1-mediated inhibition of pro-inflammatory cytokine secretion is physiologically relevant, an experiment could be conducted where Human Umbilical Vein Endothelial Cells (HUVEC) cells are exposed to wild-type B. quintana and a BepA1 mutant $B$. quintana strain. EA hy926 immortalised endothelial-like cells would also be a suitable option for this experiment and are lower-cost and easier to maintain. Given the results reported here, HUVEC cells exposed to wild-type B. quintana would be expected to secrete a lower concentration of pro-inflammatory cytokines compared to the BepA1 mutant strain, as we have demonstrated BepA1 activity in host cells reduces secretion of these cytokines. Additionally, investigating cytokine secretion by HUVEC cells exposed to BepA1 mutant B. quintana, would be interesting because it's comparable to the transfected HeLa cell cytokine quantification of this thesis but without the dsDNA from transfection stimulating a pro-inflammatory reaction. Cytokines would be quantified by ELISA to determine the concentration of each cytokine in the media; however, this concentration may not be physiologically relevant enough to alter immune cell activity. The acellular supernatant from these cells would be then transferred onto THP-1 cells, representing human macrophages, or primary human peripheral blood monocyte cells. If the difference in cytokine concentration is sufficient enough to cause activation monocyte cells from the media incubated with BepA1 mutant B. quintana, but not from wildtype B. quintana, you could conclude the BepA1-mediated pro-inflammatory cytokine inhibitory effect is physiologically relevant. This would point towards BepA1 as a significant virulence factor for the establishment of chronic B. quintana infection, allowing the bacteria to survive for longer periods of time associated with endothelial cells, hidden from the immune system. Immune cell activation could be measures by flow cytometry, identifying human leukocyte antigen as a measure of activation as well as CD62L and CD44 as a measure of migratory state and homing. Conditioned media experiments are common in studies investigating the physiological consequences of released agents. For example, Nanjundaiah et al. used conditioned media from Lactobacillus culture on J774 murine macrophages, discovering released components enhanced beneficial reactive oxygen species and decreased harmful nitrogen species build-up ${ }^{146}$. 
Chapter 6 Research Motivations, Key Findings, and Future Directions

\section{Animal Models}

Animals models could be implemented to gain a better understanding of B. quintana infection, although there is a significant cost associated. The mouse model is often used for in vivo study of pathogens; however, B. quintana does not infect mice, therefore it would not be the best choice. Rhesus macaques (Macaca mulatta) are naturally infected by B. quintana in the wild, and have been used as a pre-clinical infection model for the study of human pathogens, including B. quintana ${ }^{147}$. Experimental infection of Rhesus macaque monkeys could provide useful insight into Bartonella infection due to similarity to humans, although the disease progression differs in some respects. Experiments with the macaque model could include infecting the animal with a BepA1 mutant B. quintana strain compared to a wild-type strain and assessing the differences in disease progression. We hypothesise BepA1 is deployed by $B$. quintana to aid the bacteria in establishing chronic infection; it would be interesting to see if an inoculation by a BepA1 mutant B. quintana strain results progresses to chronic infection. Blood samples from animals infected with the wild type or a BepA1 mutant would be plated to assess the level of bacteraemia in infected animals. Alternatively, a competition experiment could be carried out, with the animal co-infected with both wild type and BepA1 mutant; the viable bacteria recovered from the animal's blood could be characterised to see if the wild type has a competitive advantage over the BepA1 mutant.

\section{BepA1-Myozap Protein Interaction Validation Studies}

Confirming the interaction between BepA1 and Myozap is essential to further research efforts discerning BepA1 activity in host cells. Cloning BepA1 into a mammalian expression vector with an attached tagging sequence, such as Myc, would provide a reliable target. A coimmunoprecipitation experiment, with proposed target, Myozap, precipitated, followed by a western blot to detecting BepA1 would support the validity of the proposed interaction.

To determine if BepA1 AMPylates Myozap via its FIC domain, the following experiment could be carried out. Myozap could be enriched from BepA1-transfected HeLa cell lysates by immunoprecipitation with an anti-Myozap antibody. Mass spectrometry could be used to detect the presence or absence of an AMP moiety on the purified Myozap protein from BepA1 transfected cells, compared to lysates from untransfected cells. In addition, mass spectrometry analysis could be used to identify the specific AMPylated Myozap residue, providing insight into the domain and downstream interactions that are interrupted. Not only would this aid in the validation of the interaction between BepA1 and Myozap, but identify the specific alternation made to Myozap by BepA1, altering its signalling capability. Determining the key 
residue targeted by BepA1 may provide some insight into the exact consequences the alteration has. To confirm this alteration is FIC domain-dependent, a site-directed BepA1 catalytic mutant could be produced, with the critical histidine residue in the conserved FIC domain mutated to an alanine residue. Immunoprecipitation and mass spectrometry analysis of Myozap could be repeated with this catalytic mutant to determine if AMPylation still occurs. If AMPylation was not observed in the presence of the catalytic mutant, it could be concluded that BepA1 AMPylates Myozap specifically at the identified residue in a FIC domain-dependent manner.

\section{BepA1-BepA2 Protein Interaction Studies}

The interaction between BepA1 and BepA2 remains uncharacterised. While researching the enzymatic activity of BepA1, the involvement of BepA2 should not be ignored. BepA1 does not contain a BID domain, essential for transportation through the VirB/D4 T4SS into host cells. BepA2 does contain a BID domain; it's possible BepA1 and BepA2 interact, complexing together for transportation through the VirB/D4 T4SS. This complexing may alter the function of either BepA1 or BepA2. The first step in answering these questions is to test for interaction between BepA1 and BepA2. A bacterial two-hybrid system could be implemented to screen for any interactions, in a similar manner as the yeast two-hybrid system was implemented during this research ${ }^{148}$.

During our research, an alternative vector was used to clone BepA2 under the low copy number Col E1 origin of replication. However, the only colonies recovered had undergone rearrangements that prevented protein expression. Due to time constraints, further cloning alternatives could not be trialled. For future cloning, ensuring the correct restriction sites are used might be enough to retrieve a successful clone. If further attempts to clone BepA2 result in recombinant events, preventing transcription, a conditional gene expression approach utilising a tightly regulated promoter would be recommended ${ }^{149}$. BepA2 could be cloned into E. coli under an inducible promoter, such as an arabinose-inducible protein, in the absence of arabinose. The promoter could be activated by the addition of arabinose, expressing BepA2 only when needed. Arabinose-inducible expression systems have become widely used in E. coli cloning; for example, Carroll et al. utilised an arabinose-inducible promoter in the investigation of Mycobacterium tuberculosis Rv1991c protein as a potent toxin ${ }^{150}$. It's also possible BepA2 functions differently in the presence of BepA1, resulting from the two proteins complexing together. 
Chapter 6 Research Motivations, Key Findings, and Future Directions

\section{HAS2 Antisense-RNA Investigation}

Another consideration stemming from the yeast two-hybrid system was the identification of the HAS2 antisense-RNA as a possible interacting partner of BepA1. Four hits were obtained identifying this interaction, strengthening its validity as each occurred independently. Due to time constraints, following up this finding was not possible during this thesis; however, this is potentially a physiologically relevant interaction that can be further investigated. HAS2 was identified from a BLAST search of the sequence recovered from the prey library plasmid. The major consideration in following up this result would be the possibility that this is an incorrectly annotated protein target, not an anti-sense RNA. If this is a cellular protein, nothing is known of its normal function, which makes it difficult to form testable hypotheses about the effect its inactivation would have on the cells. The first step would be to determine if the sequence produces a functional protein; second, we would investigate whether this protein is AMPylated by BepA1. A bioinformatics analysis of the predicted amino acid sequence could be performed using the software such as ProteinPredict, or similar software, to gain more insight into the structure and function of the protein. The goal is to determine if this sequence could form a functional protein and, if this is possible, identify it to validate the protein interaction with BepA1.

\section{NF-кB Localisation Assay}

Research into the downstream effects of BepA1 activity in host cells is an exciting future direction. Myozap interacts with SRF and MRIP; investigating changes in expression and activity of these downstream proteins in the presence of BepA1 would strengthen the validity of the interaction between BepA1 and Myozap. In addition, identifying changes in downstream transcription factor activity would help determine the cellular effects of BepA1 in host cells, driving pathogenesis. The results of this thesis showed a decrease in pro-inflammatory cytokine secretion in BepA1-transfected HeLa cells compared to untransfected cells. We theorise this decreased resulted from inhibition of the NF- $\kappa$ B signalling pathway as a downstream effect of Myozap targeting by BepA1. Once stimulated, the NF- $\mathrm{B}$ p65 subunit is released from its complex with the $\mathrm{I} \kappa \mathrm{B}$ inhibitor, and is translocated into the nucleus, where it acts as a transcription factor, stimulating expression of pro-inflammatory cytokine genes. To confirm the hypothesis that NF- $\mathrm{KB}$ is inhibited in the presence of BepA1, a localisation assay could be utilised to determine whether the NF- $\kappa \mathrm{B}$ p65 subunit moves to the nucleus following stimulation. The observed NF- $\kappa$ B inhibition either occurs by modulation of the Myozap-SRF pathway or by the Myozap-MRIP pathway leading to Rho-dependent actin modifications which prevent NF-kB entry into the nucleus. One consideration of this experiment is that the 
Chapter 6 Research Motivations, Key Findings, and Future Directions

interaction between SRF and NF- $\kappa B$ occurs via complex within the nucleus ${ }^{151}$. Therefore, it's likely that if BepA1-driven NF- $\kappa$ B inhibition occurs through the Myozap-SRF pathway, NF$\kappa \mathrm{B}$ translocation into the nucleus would not be impacted. If this were the case, typical NF- $\kappa \mathrm{B}$ translocation into the nucleus following stimulation would not dismiss the possibility that BepA1 was still driving NF- $\mathrm{BB}$ inhibition. Nuclear localisation of the transcription factor MRTF-A would also be of interest as we theorise Myozap modulation alters MRTF-A activity as well. Additionally, transcription of NF- $\kappa \mathrm{B}$ and MRTF-A in the presence of absence of BepA1, following stimulation, could be assessed utilising RT-qPCR. Downstream NF-kBdependent cytokine and SRF-dependent genes could also be assessed. Together these results would help determine the functional effects of BepA1 activity in host cells. These interesting experiments would further clarify the consequences of BepA1-driven signalling modulation within host cells, discerning the specific signalling proteins affected.

\section{ICAM-1 Whole Cell ELISA}

The results of this thesis suggest BepA1 inhibits IL- 6 and IL- 8 cytokine production by affected host cell, perhaps this action occurs through modulation of Myozap and by extension NF- $\mathrm{BB}$ and MRTF-A. One effect of MRTF-A and NF- $\kappa B$ activity on endothelial cells is the upregulation of Intercellular Adhesion Molecule 1 (ICAM-1). ICAM-1 is expressed on endothelial cells and immune cells. Cytokine stimulation results in high levels of ICAM-1 expression on the cell surface. ICAM-1 plays an important role in leukocyte binding and facilitates leukocyte endothelial transmigration, allowing leukocytes to be recruited from the blood to the site of infection. Determining if BepA1 results in downregulation of ICAM-1 would be valuable in the effort to uncover its role in pathogenesis. Human Umbilical Vein Endothelial Cells (HUVEC) could be used in future research regarding ICAM-1, representing vascular endothelial cells. HUVEC transfected with BepA1 could be compared to untransfected cells using a whole cell ELISA method. Alternatively, HeLa cells could be infected with wildtype $B$. quintana or BepA1 mutant B. quintana, then ICAM-1 quantified through whole cell ELISA. If BepA1 results in decreased ICAM-1 expression in HeLa cells, the whole cell ELISA will detect the decreased cell surface ICAM-1, compared to untransfected cells. Decreased ICAM-1 on endothelial cells would reduce immune cell adhesion, inhibiting the recruitment of neutrophils to the site of infection. This would identify another immune suppression mechanism of $B$. quintana, to better understand how chronic infection is established. 


\subsection{Concluding Remarks}

Bartonella cause infectious disease in humans, often in homeless and immune compromised individuals. Understanding the interactions between Bartonella and host cells is pivotal for the development and improvement of diagnostic techniques and treatments. The survival of $B$. quintana during early stages of infection is dependent on numerous immune evasion and possibly suppression mechanisms that ultimately allow the bacteria to disseminate and cause disease. The cellular interactions between B. quintana and the human immune system are largely unknown, and the survival of B. quintana in the presence of human immune cells is essential for the bacteria. This research aimed to investigate the interactions between $B$. quintana and human macrophages encountered in the skin during the early stages of infection. We present novel findings; we discovered human macrophages take up B. quintana poorly, identifying this as a possible immune evasion interaction. We further identified how B. quintana pathogenically modulates host cell signalling though the Bartonella effector protein, BepA1. Myozap was identified as a potential host cell target of BepA1, and the downstream cellular effects of BepA1 exposure were explored. This revealed BepA1 downregulates the host cell immune response through inhibition of pro-inflammatory cytokine secretion. While further experiments are required to validate and expand on these findings, this research advances the field of Bartonella quintana study, providing much-needed insight into the early stages of infection and how the pathogen interacts with the immune system. With this enhanced understanding of how B. quintana establishes chronic infection, further research could develop new techniques for diagnosis and treatment. 


\section{References}

1 Jones, K. E., Patel, N. G., Levy, M. A., Storeygard, A., Balk, D., Gittleman, J. L. \& Daszak, P. Global Trends in Emerging Infectious Diseases. Nature 451, 990-993, (2008).

2 Kosoy, M., Hayman, D. T. \& Chan, K. S. Bartonella bacteria in Nature: Where does Population Variability End and a Species Start? Infection, Genetics and Evolution 12, 894-904, (2012).

3 Maggi, R. G., Mascarelli, P. E., Pultorak, E. L., Hegarty, B. C., Bradley, J. M., Mozayeni, B. R. \& Breitschwerdt, E. B. Bartonella spp. Bacteremia in High-risk Immunocompetent Patients. Diagnostic Microbiology and Infectious Disease 71, 430-437, (2011).

Angelakis, E. \& Raoult, D. Pathogenicity and Treatment of Bartonella Infections. International Journal of Antimicrobial Agents 44, 16-25, (2014).

5 Minnick, M. F. \& Anderson, B. E. Molecular Medical Microbiology: Chapter 105 Bartonella. Second edn, (Academic Press, 2015).

Fournier, P. E., Taylor, C., Rolain, J. M., Barrassi, L., Smith, G. \& Raoult, D. Bartonella australis sp. nov. from Kangaroos, Australia. Emerging Infectious Diseases 13, 1961-1962, (2007).

Carrasco, S. E., Chomel, B. B., Gill, V. A., Kasten, R. W., Maggi, R. G., Breitschwerdt, E. B., Byrne, B. A., Burek-Huntington, K. A., Miller, M. A., Goldstein, T. \& Mazet, J. A. K. Novel Bartonella Infection in Northern and Southern Sea Otters (Enhydra lutris kenyoni and Enhydra lutris nereis). Veterinary Microbiology 170, 325-334, (2014).

Chomel, B. B., Kasten, R. W., Sykes, J. E., Boulouis, H. J. \& Breitschwerdt, E. B. Clinical Impact of Persistent Bartonella Bacteremia in Humans and Animals. Annals of the New York Academy of Sciences 990, 267-278, (2003).

Eicher, S. C. \& Dehio, C. Bartonella Entry Mechanisms into Mammalian Host Cells. Cellular Microbiology 14, 1166-1173, (2012).

10 Boulouis, H. J., Chang, C. C., Henn, J. B., Kasten, R. W. \& Chomel, B. B. Factors Associated with the Rapid Emergence of Zoonotic Bartonella Infections. Veterinary Research 36, 383-410, (2005).

11 Guy, L., Nystedt, B., Toft, C., Zaremba-Niedzwiedzka, K., Berglund, E. C., Granberg, F., Näslund, K., Eriksson, A.-S. \& Andersson, S. G. E. A Gene Transfer Agent and a Dynamic Repertoire of Secretion Systems Hold the Keys to the Explosive Radiation of the Emerging Pathogen Bartonella. PLOS Genetics 9, e1003393, (2013).

12 Harms, A. \& Dehio, C. Intruders Below the Radar: Molecular Pathogenesis of Bartonella spp. Clinical Microbiology Reviews 25, 42-78, (2012).

13 Sanchez Clemente, N., Ugarte-Gil, C. A., Solórzano, N., Maguiña, C., Pachas, P., Blazes, D., Bailey, R., Mabey, D. \& Moore, D. Bartonella bacilliformis: A Systematic Review of the Literature to Guide the Research Agenda for Elimination. Plos Neglected Tropical Diseases 6, e1819, (2012).

14 Maguiña, C., Guerra, H. \& Ventosilla, P. Bartonellosis. Clinics in Dermatology 27, 271-280, (2009). 
References

15 Allison, M. J., Pezzia, A., Gerszten, E. \& Mendoza, D. A Case of Carrion's Disease Associated with Human Sacrifice from the Huari Culture of Southern Peru. American Journal of Physical Anthropology 41, 295-300, (1974).

16 Breitschwerdt, E. B. Bartonellosis: One Health Perspectives for an Emerging Infectious Disease. ILAR Journal 55, 46-58, (2014).

17 Chomel, B. B., Kasten, R. W., Floyd-Hawkins, K., Chi, B., Yamamoto, K., Roberts-Wilson, J., Gurfield, A. N., Abbott, R. C., Pedersen, N. C. \& Koehler, J. E. Experimental Transmission of Bartonella henselae by the Cat Flea. Journal of Clinical Microbiology 34, 1952-1956, (1996).

18 Foil, L., Andress, E., Freeland, R. L., Roy, A. F., Rutledge, R., Triche, P. C. \& O'Reilly, K. L. Experimental Infection of Domestic Cats with Bartonella henselae by Inoculation of Ctenocephalides felis (Siphonaptera: Pulicidae) Feces. Journal of Medical Entomology 35, 625-628, (1998).

19 Stutzer, B. \& Hartmann, K. Chronic Bartonellosis in Cats What are the Potential Implications? Journal of Feline Medicine and Surgery 14, 612-621, (2012).

20 Kordick, D. L. \& Breitschwerdt, E. B. Intraerythrocytic Presence of Bartonella henselae. Journal of Clinical Microbiology 33, 1655-1656, (1995).

21 Kordick, D. L., Wilson, K. H., Sexton, D. J., Hadfield, T. L., Berkhoff, H. A. \& Breitschwerdt, E. B. Prolonged Bartonella Bacteremia in Cats Associated with Cat-scratch Disease Patients. Journal of Clinical Microbiology 33, 3245-3251, (1995).

22 Kordick, D. L., Brown, T. T., Shin, K. \& Breitschwerdt, E. B. Clinical and Pathologic Evaluation of Chronic Bartonella henselae or Bartonella clarridgeiae Infection in Cats. Journal of Clinical Microbiology 37, 1536-1547, (1999).

23 Nelson, C. A., Saha, S. \& Mead, P. S. Cat-Scratch Disease in the United States, 2005-2013. Emerging Infectious Diseases 22, 1741-1746, (2016).

24 Lantos, P. M., Maggi, R. G., Ferguson, B., Varkey, J., Park, L. P., Breitschwerdt, E. B. \& Woods, C. W. Detection of Bartonella Species in the Blood of Veterinarians and Veterinary Technicians: a Newly Recognized Occupational Hazard? Vector-Borne and Zoonotic Diseases 14, 563-570, (2014).

25 Mosepele, M., Mazo, D. \& Cohn, J. Bartonella Infection in Immunocompromised Hosts: Immunology of Vascular Infection and Vasoproliferation. Clin Dev Immunol 2012, (2012).

26 Atenstaedt, R. L. Trench Fever: the British Medical Response in the Great War. Journal of the Royal Society of Medicine 99, 564-568, (2006).

27 Anstead, G. M. The Centenary of the Discovery of Trench Fever, an Emerging Infectious Disease of World War 1. The Lancet Infectious Diseases 16, 164-172, (2016).

28 Wright, T. S. Some Notes on Trench Fever. The British Medical Journal 2, 136-138, (1916).

29 Maurin, M. \& Raoult, D. Bartonella (Rochalimaea) quintana infections. Clinical Microbiology Reviews 9, 273-292, (1996).

30 O'Connor, S. P., Dorsch, M., Steigerwalt, A. G., Brenner, D. J. \& Stackebrandt, E. 16S rRNA Sequences of Bartonella bacilliformis and Cat Scratch Disease Bacillus Reveal Phylogenetic Relationships with the Alpha-2 Subgroup of the Class Proteobacteria. Journal of Clinical Microbiology 29, 2144-2150, (1991). 


\section{References}

31 Raoult, D., Dutour, O., Houhamdi, L., Jankauskas, R., Fournier, P. E., Ardagna, Y., Drancourt, M., Signoli, M., La, V. D., Macia, Y. \& Aboudharam, G. Evidence for Louse-transmitted Diseases in Soldiers of Napoleon's Grand Army in Vilnius. The Journal of Infectious Diseases 193, 112-120, (2006).

32 Nguyen-Hieu, T., Aboudharam, G., Signoli, M., Rigeade, C., Drancourt, M. \& Raoult, D. Evidence of a Louse-borne Outbreak Involving Typhus in Douai, 1710-1712 During the War of Spanish Succession. PLoS One 5, e15405, (2010).

33 Drancourt, M., Tran-Hung, L., Courtin, J., Lumley, H. \& Raoult, D. Bartonella quintana in a 4000-year-old Human Tooth. The Journal of Infectious Diseases 191, 607-611, (2005).

34 Ohl, M. E. \& Spach, D. H. Bartonella quintana and Urban Trench Fever. Clinical Infectious Diseases 31, 131-135, (2000).

35 Cutler, S., Abdissa, A., Adamu, H., Tolosa, T. \& Gashaw, A. Bartonella quintana in Ethiopian Lice. Comparative Immunology Microbiology and Infectious Diseases 35, 17-21, (2012).

36 Sasaki, T., Poudel, S. K., Isawa, H., Hayashi, T., Seki, N., Tomita, T., Sawabe, K. \& Kobayashi, M. First Molecular Evidence of Bartonella quintana in Pediculus humanus capitis (Phthiraptera: Pediculidae), Collected from Nepalese Children. Journal of Medical Entomology 43, 110-112, (2006).

37 Telford, S. R. \& Wormser, G. P. Bartonella spp. Transmission by Ticks Not Established. Emerging Infectious Diseases 16, 379-384, (2010).

38 Byam, W., Carroll, J. H., Churchill, J. H., Dimond, L., Sorapure, V. E., Wilson, R. M. \& Lloyd, L. L. Trench Fever. A Louse-Borne Disease. 237-284 (Jf. Krowdc, Hodder \& Stoughton, Oxford University Press., 1919).

39 Bonilla, D. L., Cole-Porse, C., Kjemtrup, A., Osikowicz, L. \& Kosoy, M. Risk Factors for Human Lice and Bartonellosis Among the Homeless, San Francisco, California, USA. Emerging Infectious Diseases 20, 1645-1651, (2014).

40 Karem, K. L., Paddock, C. D. \& Regnery, R. L. Bartonella henselae, B. quintana, and B. bacilliformis: Historical Pathogens of Emerging Significance. Microbes and Infection 2, 11931205, (2000).

41 Cédric, F., Philippe, B. \& Didier, R. Bartonella quintana Characteristics and Clinical Management. Emerging Infectious Disease journal 12, 217-223, (2006).

42 Spach, D. H., Kanter, A. S., Dougherty, M. J., Larson, A. M., Coyle, M. B., Brenner, D. J., Swaminathan, B., Matar, G. M., Welch, D. F. \& Root, R. K. Bartonella (Rochalimaea) quintana Bacteremia in Inner-city Patients with Chronic Alcoholism. The New England Journal of Medicine 332, 424-428, (1995).

43 Foucault, C., Barrau, K., Brouqui, P. \& Raoult, D. Bartonella quintana Bacteremia Among Homeless People. Clinical Infectious Diseases 35, 684-689, (2002).

44 Cahill, T. J. \& Prendergast, B. D. Infective Endocarditis. The Lancet 387, 882-893.

45 Naber, C. K. \& Erbel, R. Diagnosis of Culture Negative Endocarditis: Novel Strategies to Prove the Suspect Guilty. Heart 89, 241-243, (2003).

46 Drancourt, M., Mainardi, J. L., Brouqui, P., Vandenesch, F., Carta, A., Lehnert, F., Etienne, J., Goldstein, F., Acar, J. \& Raoult, D. Bartonella (Rochalimaea) quintana Endocarditis in Three Homeless Men. The New England Journal of Medicine 332, 419-423, (1995). 


\section{References}

47 Spach, D. H., Kanter, A. S., Daniels, N. A., Nowowiejski, D. J., Larson, A. M., Schmidt, R. A., Swaminathan, B. \& Brenner, D. J. Bartonella (Rochalimaea) Species as a Cause of Apparent "Culture-negative" Endocarditis. Clinical Infectious Diseases 20, 1044-1047, (1995).

48 Raoult, D., Fournier, P. E., Drancourt, M., Marrie, T. J., Etienne, J., Cosserat, J., Cacoub, P., Poinsignon, Y., Leclercq, P. \& Sefton, A. M. Diagnosis of 22 New Cases of Bartonella endocarditis. Annals of Internal Medicine 125, 646-652, (1996).

49 Spach, D. H., Callis, K. P., Paauw, D. S., Houze, Y. B., Schoenknecht, F. D., Welch, D. F., Rosen, H. \& Brenner, D. J. Endocarditis Caused by Rochalimaea quintana in a Patient Infected with Human Immunodeficiency Virus. Journal of Clinical Microbiology 31, 692-694, (1993).

50 Fournier, P. E., Lelievre, H., Eykyn, S. J., Mainardi, J. L., Marrie, T. J., Bruneel, F., Roure, C., Nash, J., Clave, D., James, E., Benoit-Lemercier, C., Deforges, L., Tissot-Dupont, H. \& Raoult, D. Epidemiologic and Clinical Characteristics of Bartonella quintana and Bartonella henselae Endocarditis: a Study of 48 Patients. Medicine 80, 245-251, (2001).

51 Fournier, P. E., Thuny, F., Richet, H., Lepidi, H., Casalta, J. P., Arzouni, J. P., Maurin, M., Celard, M., Mainardi, J. L., Caus, T., Collart, F., Habib, G. \& Raoult, D. Comprehensive Diagnostic Strategy for Blood Culture-negative Endocarditis: a Prospective Study of $819 \mathrm{New}$ Cases. Clinical Infectious Diseases 51, 131-140, (2010).

La Scola, B. \& Raoult, D. Serological Cross-reactions Between Bartonella quintana, Bartonella henselae, and Coxiella burnetii. Journal of Clinical Microbiology 34, 2270-2274, (1996). 937-962, (2002). Recommendations for Treatment of Human Infections Caused by Bartonella Species. Antimicrobial Agents and Chemotherapy 48, 1921-1933, (2004).

Foucault, C., Raoult, D. \& Brouqui, P. Randomized Open Trial of Gentamicin and Doxycycline for Eradication of Bartonella quintana from Blood in Patients with Chronic Bacteremia. Antimicrobial Agents and Chemotherapy 47, 2204-2207, (2003).

56 Kyme, P. A., Haas, A., Schaller, M., Peschel, A., Iredell, J. \& Kempf, V. A. Unusual Trafficking Pattern of Bartonella henselae-containing Vacuoles in Macrophages and Endothelial Cells. Cellular Microbiology 7, 1019-1034, (2005).

57 Okujava, R., Guye, P., Lu, Y. Y., Mistl, C., Polus, F., Vayssier-Taussat, M., Halin, C., Rolink, A. G. \& Dehio, C. A Translocated Effector Required for Bartonella Dissemination from Derma to Blood Safeguards Migratory Host Cells from Damage by Co-translocated Effectors. Plos Pathogens 10, e1004187, (2014).

58 Schulein, R., Seubert, A., Gille, C., Lanz, C., Hansmann, Y., Piemont, Y. \& Dehio, C. Invasion and Persistent Intracellular Colonization of Erythrocytes. A Unique Parasitic Strategy of the Emerging Pathogen Bartonella. Journal of Experimental Medicine 193, 1077-1086, (2001).

59 Dehio, C. Molecular and Cellular Basis of Bartonella Pathogenesis. Annual Review of Microbiology 58, 365-390, (2004).

60 Hong, J., Li, Y., Hua, X., Bai, Y., Wang, C., Zhu, C., Du, Y., Yang, Z. \& Yuan, C. Lymphatic Circulation Disseminates Bartonella Infection Into Bloodstream. The Journal of Infectious Diseases 215, 303-311, (2017).

61 Dehio, C. Bartonella-host-cell Interactions and Vascular Tumour Formation. Nature Reviews Microbiology 3, 621-631, (2005). 


\section{References}

62 Koesling, J., Aebischer, T., Falch, C., Schulein, R. \& Dehio, C. Cutting Edge: Antibodymediated Cessation of Hemotropic Infection by the Intraerythrocytic Mouse Pathogen Bartonella grahamii. Journal of Immunology 167, (2001).

63 Minnick, M. F. \& Battisti, J. M. Pestilence, Persistence and Pathogenicity: Infection Strategies of Bartonella. Future Microbiology 4, 743-758, (2009).

64 Chiaraviglio, L., Duong, S., Brown, D. A., Birtles, R. J. \& Kirby, J. E. An Immunocompromised Murine Model of Chronic Bartonella Infection. The American Journal of Pathology 176, 27532763, (2010).

65 Popa, C., Abdollahi-Roodsaz, S., Joosten, L. A. B., Takahashi, N., Sprong, T., Matera, G., Liberto, M. C., Foca, A., van Deuren, M., Kullberg, B. J., van den Berg, W. B., van der Meer, J. W. M. \& Netea, M. G. Bartonella quintana Lipopolysaccharide Is a Natural Antagonist of Toll-Like Receptor 4. Infection and Immunity 75, 4831-4837, (2007).

Capo, C., Amirayan-Chevillard, N., Brouqui, P., Raoult, D. \& Mege, J. L. Bartonella quintana Bacteremia and Overproduction of Interleukin-10: Model of Bacterial Persistence in Homeless People. The Journal of Infectious Diseases 187, 837-844, (2003).

Pappalardo, B. L., Brown, T. T., Tompkins, M. \& Breitschwerdt, E. B. Immunopathology of Bartonella vinsonii (berkhoffii) in Experimentally Infected Dogs. Veterinary Immunology and Immunopathology 83, 125-147, (2001). Bartonella Species to their Hosts and Vectors. Veterinary Research 40, (2009).

70 Vayssier-Taussat, M., Le Rhun, D., Deng, H. K., Biville, F., Cescau, S., Danchin, A., Marignac, G., Lenaour, E., Boulouis, H. J., Mavris, M., Arnaud, L., Yang, H., Wang, J., Quebatte, M., Engel, P., Saenz, H. \& Dehio, C. The Trw Type IV Secretion System of Bartonella Mediates Host-specific Adhesion to Erythrocytes. Plos Pathogens 6, e1000946, (2010).

71 Deng, H. K., Le Rhun, D., Le Naour, E., Bonnet, S. \& Vayssier-Taussat, M. Identification of Bartonella Trw Host-Specific Receptor on Erythrocytes. PLoS One 7, e41447, (2012).

Alsmark, C. M., Frank, A. C., Karlberg, E. O., Legault, B. A., Ardell, D. H., Canback, B., Eriksson, A. S., Naslund, A. K., Handley, S. A., Huvet, M., La Scola, B., Holmberg, M. \& Andersson, S. G. The Louse-borne Human Pathogen Bartonella quintana is a Genomic Derivative of the Zoonotic Agent Bartonella henselae. Proceedings of the National Academy of Sciences of the USA 101, 9716-9721, (2004).

73 Zhou, L., Tan, A. \& Hershenson, M. B. Yersinia YopJ Inhibits Pro-inflammatory Molecule Expression in Human Bronchial Epithelial Cells. Respiratory Physiology \& Neurobiology 140, 89-97, (2004).

74 Iwaki-Egawa, S. \& Ihler, G. M. Comparison of the Abilities of Proteins from Bartonella bacilliformis and Bartonella henselae to Deform Red Cell Membranes and to Bind to Red Cell Ghost Proteins. FEMS Microbiology Letters 157, 207-217, (1997).

75 Xu, Y.-H., Lu, Z.-Y. \& Ihler, G. M. Purification of Deformin, an Extracellular Protein Synthesized by Bartonella bacilliformis Which Causes Deformation of Erythrocyte Membranes. BBA Biomembranes 1234, 173-183, (1995). 
77 Quebatte, M., Dehio, M., Tropel, D., Basler, A., Toller, I., Raddatz, G., Engel, P., Huser, S., Schein, H., Lindroos, H. L., Andersson, S. G. \& Dehio, C. The BatR/BatS Two-component Regulatory System Controls the Adaptive Response of Bartonella henselae During Human Endothelial Cell Infection. Journal of Bacteriology 192, 3352-3367, (2010).

78 Zhang, P., Chomel, B. B., Schau, M. K., Goo, J. S., Droz, S., Kelminson, K. L., George, S. S., Lerche, N. W. \& Koehler, J. E. A Family of Variably Expressed Outer-membrane Proteins (Vomp) Mediates Adhesion and Autoaggregation in Bartonella quintana. Proceedings of the National Academy of Sciences of the USA 101, 13630-13635, (2004).

79 Müller, N. F., Kaiser, P. O., Linke, D., Schwarz, H., Riess, T., Schäfer, A., Eble, J. A. \& Kempf, V. A. J. Trimeric Autotransporter Adhesin-Dependent Adherence of Bartonella henselae, Bartonella quintana, and Yersinia enterocolitica to Matrix Components and Endothelial Cells under Static and Dynamic Flow Conditions. Infection and Immunity 79, 2544-2553, (2011).

80 Schulte, B., Linke, D., Klumpp, S., Schaller, M., Riess, T., Autenrieth, I. B. \& Kempf, V. A. J. Bartonella quintana Variably Expressed Outer Membrane Proteins Mediate Vascular Endothelial Growth Factor Secretion but Not Host Cell Adherence. Infection and Immunity 74, 5003-5013, (2006).

81 Scherer, D. C., DeBuron-Connors, I. \& Minnick, M. F. Characterization of Bartonella bacilliformis Flagella and Effect of Antiflagellin Antibodies on Invasion of Human Erythrocytes. Infection and Immunity 61, 4962-4971, (1993).

82 Wallden, K., Rivera-Calzada, A. \& Waksman, G. Type IV Secretion Systems: Versatility and Diversity in Function. Cellular Microbiology 12, 1203-1212, (2010).

83 Schroder, G., Schuelein, R., Quebatte, M. \& Dehio, C. Conjugative DNA Transfer into Human Cells by the VirB/VirD4 Type IV Secretion System of the Bacterial Pathogen Bartonella henselae. Proceedings of the National Academy of Sciences of the USA 108, 14643-14648, (2011).

84 Pulliainen, A. T. \& Dehio, C. Bartonella henselae: Subversion of Vascular Endothelial Cell Functions by Translocated Bacterial Effector Proteins. The International Journal of Biochemistry \& Cell Biology 41, 507-510, (2009).

85 Siamer, S. \& Dehio, C. New Insights into the Role of Bartonella Effector Proteins in Pathogenesis. Current Opinion in Microbiology 23, 80-85, (2015).

Schulein, R., Guye, P., Rhomberg, T. A., Schmid, M. C., Schroder, G., Vergunst, A. C., Carena, I. \& Dehio, C. A Bipartite Signal Mediates the Transfer of Type IV Secretion Substrates of Bartonella henselae into Human Cells. Proceedings of the National Academy of Sciences of the USA 102, 856-861, (2005).

87 Schmid, M. C., Schulein, R., Dehio, M., Denecker, G., Carena, I. \& Dehio, C. The VirB Type IV Secretion System of Bartonella henselae Mediates Invasion, Proinflammatory Activation and Antiapoptotic Protection of Endothelial Cells. Molecular Microbiology 52, 81-92, (2004).

Schmid, M. C., Scheidegger, F., Dehio, M., Balmelle-Devaux, N., Schulein, R., Guye, P., Chennakesava, C. S., Biedermann, B. \& Dehio, C. A Translocated Bacterial Protein Protects Vascular Endothelial Cells from Apoptosis. Plos Pathogens 2, 1083-1097, (2006).

89 Scheidegger, F., Ellner, Y., Guye, P., Rhomberg, T. A., Weber, H., Augustin, H. G. \& Dehio, C. Distinct Activities of Bartonella henselae Type IV Secretion Effector Proteins Modulate Capillary-like Sprout Formation. Cellular Microbiology 11, 1088-1101, (2009).

90 Harms, A., Segers, F. H. I. D., Quebatte, M., Mistl, C., Manfredi, P., Körner, J., Chomel, B. B., Kosoy, M., Maruyama, S., Engel, P. \& Dehio, C. Evolutionary Dynamics of Pathoadaptation 
References

Revealed by Three Independent Acquisitions of the VirB/D4 Type IV Secretion System in Bartonella. Genome Biology and Evolution 9, 761-776, (2017).

91 Rhomberg, T. A., Truttmann, M. C., Guye, P., Ellner, Y. \& Dehio, C. A Translocated Protein of Bartonella henselae Interferes with Endocytic Uptake of Individual Bacteria and Triggers Uptake of Large Bacterial Aggregates via the Invasome. Cellular Microbiology 11, 927-945, (2009).

92 Roy, C. R. \& Mukherjee, S. Bacterial FIC Proteins AMP Up Infection. Sci Signal 2, 1-3, (2009).

93 Pulliainen, A. T., Pieles, K., Brand, C. S., Hauert, B., Böhm, A., Quebatte, M., Wepf, A., Gstaiger, M., Aebersold, R., Dessauer, C. W. \& Dehio, C. Bacterial Effector Binds Host Cell Adenylyl Cyclase to Potentiate Gas-dependent cAMP production. Proceedings of the National Academy of Sciences of the USA 109, 9581-9586, (2012).

94 Stadtman, E. R., Ginsburg, A., Ciardi, J. E., Yeh, J., Hennig, S. B. \& Shapiro, B. M. Multiple Molecular Forms of Glutamine Synthetase Produced by Enzyme Catalyzed Adenylation and Deadenylylation Reactions. Adv Enzyme Regul 8, 99-118, (1970).

95 Brown, M. S., Segal, A. \& Stadtman, E. R. Modulation of Glutamine Synthetase Adenylylation and Deadenylylation Is Mediated by Metabolic Transformation of the P(II)-Regulatory Protein. Proceedings of the National Academy of Sciences of the USA 68, 2949-2953, (1971).

96 Worby, C. A., Mattoo, S., Kruger, R. P., Corbeil, L. B., Koller, A., Mendez, J. C., Zekarias, B., Lazar, C. \& Dixon, J. E. The Fic Domain: A New Paradigm for Adenylylation. Molecular Cell 34, 93-103, (2009).

97 Engel, P., Goepfert, A., Stanger, F. V., Harms, A., Schmidt, A., Schirmer, T. \& Dehio, C. Adenylylation Control by Intra- or Intermolecular Active-site Obstruction in Fic Proteins. Nature 482, 107-110, (2012).

98 Pieles, K., Glatter, T., Harms, A., Schmidt, A. \& Dehio, C. An Experimental Strategy for the Identification of AMPylation Targets from Complex Protein Samples. Proteomics 14, 10481052 , (2014).

99 Regnery, R. L., Anderson, B. E., Clarridge Iii, J. E., Rodriguez-Barradas, M. C., Jones, D. C. \& Carr, J. H. Characterization of a Novel Rochalimaea species, $R$. henselae sp. nov., Isolated from Blood of a Febrile, Human Immunodeficiency Virus-positive Patient. Journal of Clinical Microbiology 30, 265-274, (1992).

100 Musso, T., Badolato, R., Ravarino, D., Stornello, S., Panzanelli, P., Merlino, C., Savoia, D., Cavallo, R., Ponzi, A. N. \& Zucca, M. Interaction of Bartonella henselae with the Murine Macrophage Cell Line J774: Infection and Proinflammatory Response. Infection and Immunity 69, 5974-5980, (2001).

101 Flannagan, R. S., Heit, B. \& Heinrichs, D. E. Intracellular Replication of Staphylococcus aureus in Mature Phagolysosomes in Macrophages Precedes Host Cell Death, and Bacterial Escape and Dissemination. Cellular Microbiology 18, 514-535, (2016).

102 Arabski, M., Wegierek-Ciuk, A., Czerwonka, G., Lankoff, A. \& Kaca, W. Effects of Saponins Against Clinical E. coli Strains and Eukaryotic Cell Line. Journal of Biomedicine and Biotechnology 2012, (2012).

103 Subashchandrabose, S., Smith, S. N., Spurbeck, R. R., Kole, M. M. \& Mobley, H. L. Genomewide Detection of Fitness Genes in Uropathogenic Escherichia coli During Systemic Infection. Plos Pathogens 9, e1003788, (2013). 
104 Dehio, C., Meyer, M., Berger, J., Schwarz, H. \& Lanz, C. Interaction of Bartonella henselae with Endothelial Cells Results in Bacterial Aggregation on the Cell Surface and the Subsequent Engulfment and Internalisation of the Bacterial Aggregate by a Unique Structure, the Invasome. Journal of Cell Science 110 ( Pt 18), 2141-2154, (1997).

105 Truttmann, M. C., Rhomberg, T. A. \& Dehio, C. Combined Action of the Type IV Secretion Effector Proteins BepC and BepF Promotes Invasome Formation of Bartonella henselae on Endothelial and Epithelial Cells. Cellular Microbiology 13, 284-299, (2011).

106 Malgorzata-Miller, G., Heinbockel, L., Brandenburg, K., van der Meer, J. W., Netea, M. G. \& Joosten, L. A. Bartonella quintana Lipopolysaccharide (LPS): Structure and Characteristics of a Potent TLR4 antagonist for in-vitro and in-vivo Applications. Scientific Reports 6, 34221, (2016).

107 Johnson, M. B. \& Criss, A. K. Fluorescence Microscopy Methods for Determining the Viability of Bacteria in Association with Mammalian Cells. Journal of Visualized Experiments : JoVE, e50729 (2013).

108 Riess, T., Andersson, S. G. E., Lupas, A., Schaller, M., Schäfer, A., Kyme, P., Martin, J., Wälzlein, J.-H., Ehehalt, U., Lindroos, H., Schirle, M., Nordheim, A., Autenrieth, I. B. \& Kempf, V. A. J. Bartonella Adhesin A Mediates a Proangiogenic Host Cell Response. Journal of Experimental Medicine 200, 1267-1278, (2004).

109 China, B., N'Guyen, B. T., de Bruyere, M. \& Cornelis, G. R. Role of YadA in Resistance of Yersinia enterocolitica to Phagocytosis by Human Polymorphonuclear Leukocytes. Infection and Immunity 62, 1275-1281, (1994).

110 Popa, C. M., Tabuchi, M. \& Valls, M. Modification of Bacterial Effector Proteins Inside Eukaryotic Host Cells. Frontiers in Cellular and Infection Microbiology 6, (2016).

111 Galán, J. E. Common Themes in the Design and Function of Bacterial Effectors. Cell Host \& Microbe 5, 571-579, (2009).

112 Ke, Y., Chen, Z. \& Yang, R. Yersinia pestis: Mechanisms of Entry into and Resistance to the Host Cell. Frontiers in Cellular and Infection Microbiology 3, (2013).

113 Yarbrough, M. L., Li, Y., Kinch, L. N., Grishin, N. V., Ball, H. L. \& Orth, K. AMPylation of Rho GTPases by Vibrio VopS Disrupts Effector Binding and Downstream Signaling. Science 323, 269-272, (2009).

114 Schmid, M. C., Scheidegger, F., Dehio, M., Balmelle-Devaux, N., Schulein, R., Guye, P., Chennakesava, C. S., Biedermann, B. \& Dehio, C. A Translocated Bacterial Protein Protects Vascular Endothelial Cells from Apoptosis. Plos Pathogens 2, e115, (2006).

115 Seeger, T. S., Frank, D., Rohr, C., Will, R., Just, S., Grund, C., Lyon, R., Luedde, M., Koegl, M., Sheikh, F., Rottbauer, W., Franke, W. W., Katus, H. A., Olson, E. N. \& Frey, N. Myozap, a Novel Intercalated Disc Protein, Activates Serum Response Factor-dependent Signaling and is Required to Maintain Cardiac Function in vivo. Circulation Research 106, 880-890, (2010).

116 Luong, P., Kinch, L. N., Brautigam, C. A., Grishin, N. V., Tomchick, D. R. \& Orth, K. Kinetic and Structural Insights into the Mechanism of AMPylation by VopS Fic Domain. Journal of Biological Chemistry 285, 20155-20163, (2010).

117 Barth, H., Fischer, S., Möglich, A. \& Förtsch, C. Clostridial C3 Toxins Target Monocytes/Macrophages and Modulate Their Functions. Frontiers in Immunology 6, (2015).

118 Schulein, R. \& Dehio, C. The VirB/VirD4 Type IV Secretion System of Bartonella is Essential for Establishing Intraerythrocytic Infection. Molecular Microbiology 46, 1053-1067, (2002). 
119 Kim, Y. J., Lin, N.-C. \& Martin, G. B. Two Distinct Pseudomonas Effector Proteins Interact with the Pto Kinase and Activate Plant Immunity. Cell 109, 589-598, (2002).

120 Bernal-Bayard, J. \& Ramos-Morales, F. Salmonella Type III Secretion Effector SlrP Is an E3 Ubiquitin Ligase for Mammalian Thioredoxin. Journal of Biological Chemistry 284, 2758727595, (2009).

121 Riddick, N., Ohtani, K. i. \& Surks, H. K. Targeting by Myosin Phosphatase-RhoA Interacting Protein Mediates RhoA/ROCK Regulation of Myosin Phosphatase. Journal of Cellular Biochemistry 103, 1158-1170, (2008).

122 Surks, H. K., Richards, C. T. \& Mendelsohn, M. E. Myosin Phosphatase-Rho Interacting Protein: A New Member of the Myosin Phosphatase Complex that Directly Binds RhoA. Journal of Biological Chemistry 278, 51484-51493, (2003).

123 Yu, L., Weng, X., Liang, P., Dai, X., Wu, X., Xu, H., Fang, M., Fang, F. \& Xu, Y. MRTF-A Mediates LPS-induced Pro-inflammatory Transcription by Interacting with the COMPASS Complex. Journal of Cell Science 127, 4645-4657, (2014).

124 Sharif, O., Bolshakov, V. N., Raines, S., Newham, P.\& Perkins, N. D. Transcriptional Profiling of the LPS Induced NF-kappaB Response in Macrophages. BMC Immunol 8, (2007).

125 Roebuck, K. A. \& Finnegan, A. Regulation of Intercellular Adhesion Molecule-1 (CD54) Gene Expression. Journal of Leukocyte Biology 66, 876-888, (1999).

126 Hayashi, K., Murai, T., Oikawa, H., Masuda, T., Kimura, K., Muehlich, S., Prywes, R. \& Morita, T. A Novel Inhibitory Mechanism of MRTF-A/B on the ICAM-1 Gene Expression in Vascular Endothelial Cells. Scientific Reports 5, 10627, (2015).

127 Wesslen, L., Ehrenborg, C., Holmberg, M., McGill, S., Hjelm, E., Lindquist, O., Henriksen, E., Rolf, C., Larsson, E. \& Friman, G. Subacute Bartonella Infection in Swedish Orienteers Succumbing to Sudden Unexpected Cardiac Death or Having Malignant Arrhythmias. Scandinavian Journal of Infectious Diseases 33, 429-438, (2001).

128 McGill, S., Wesslen, L., Hjelm, E., Holmberg, M., Rolf, C. \& Friman, G. Serological and Epidemiological Analysis of the Prevalence of Bartonella spp. Antibodies in Swedish Elite Orienteers 1992-93. Scandinavian Journal of Infectious Diseases 33, 423-428, (2001).

129 Santilli, R. A., Battaia, S., Perego, M., Tursi, M., Grego, E., Marzufero, C. \& Gianella, P. Bartonella-associated Inflammatory Cardiomyopathy in a Dog. Journal of Veterinary Cardiology 19, 74-81, (2017).

130 Forbes, M. S. \& Sperelakis, N. Intercalated Discs of Mammalian Heart: a Review of Structure and Function. Cell Tissue Res 17, 605-648, (1985).

131 Rickelt, S., Kuhn, C., Winter-Simanowski, S., Zimbelmann, R., Frey, N. \& Franke, W. W. Protein Myozap - a Late Addition to the Molecular Ensembles of Various Kinds of Adherens Junctions. Cell Tissue Res 346, 347-359, (2011).

132 Desai, L. P., Aryal, A. M., Ceacareanu, B., Hassid, A. \& Waters, C. M. RhoA and Rac1 are Both Required for Efficient Wound Closure of Airway Epithelial Cells. American Journal of Physiology-Lung Cellular and Molecular Physiology 287, 1134-1144, (2004).

133 Johannessen, M., Askarian, F., Sangvik, M. \& Sollid, J. E. Bacterial Interference with Canonical NFкB Signalling. Microbiology 159, 2001-2013, (2013).

134 Bugalhão, J. N., Mota, L. J. \& Franco, I. S. Bacterial Nucleators: Actin' on Actin. Pathogens and Disease 73, (2015). 
135 Popoff, M. R. Bacterial Factors Exploit Eukaryotic Rho GTPase Signaling Cascades to Promote Invasion and Proliferation within their Host. Small GTPases 5, e28209, (2014).

136 McLaughlin, L. M., Govoni, G. R., Gerke, C., Gopinath, S., Peng, K., Laidlaw, G., Chien, Y. H., Jeong, H. W., Li, Z. G., Brown, M. D., Sacks, D. B. \& Monack, D. The Salmonella SPI2 Effector SseI Mediates Long-Term Systemic Infection by Modulating Host Cell Migration. Plos Pathogens 5, e1000671, (2009).

137 Brothers, K. M., Stella, N. A., Hunt, K. M., Romanowski, E. G., Liu, X., Klarlund, J. K. \& Shanks, R. M. Q. Putting on the Brakes: Bacterial Impediment of Wound Healing. Scientific Reports 5, 14003, (2015).

138 Vinson, J. W., Varela, G. \& Molina-Pasquel, C. Trench Fever. Induction of Clinical Disease in Volunteers Inoculated with Rickettsia quintana Propagated on Blood Agar. The American Journal of Tropical Medicine and Hygiene 18, 713-722, (1969).

139 Vinson, J. W. In vitro Cultivation of the Rickettsial Agent of Trench Fever. Bulletin of the World Health Organization 35, 155-164, (1966).

140 Liberto, M. C., Matera, G., Lamberti, A. G., Barreca, G. S., Foca, D., Quirino, A., Soria, M. R. \& Foca, A. Bartonella quintana-induced Apoptosis Inhibition of Human Endothelial Cells is Associated with p38 and SAPK/JNK Modulation and with Stimulation of Mitosis. Diagnostic Microbiology and Infectious Disease 50, 159-166, (2004).

141 Dehio, C. Bartonella Interactions with Endothelial Cells and Erythrocytes. Trends in Microbiology 9, 279-285, (2001).

142 Breitschwerdt, E. B., Maggi, R. G., Nicholson, W. L., Cherry, N. A. \& Woods, C. W. Bartonella sp. Bacteremia in Patients with Neurological and Neurocognitive Dysfunction. Journal of Clinical Microbiology 46, 2856-2861, (2008).

143 Breitschwerdt, E. B., Maggi, R. G., Lantos, P. M., Woods, C. W., Hegarty, B. C. \& Bradley, J. M. Bartonella vinsonii subsp. berkhoffii and Bartonella henselae Bacteremia in a Father and Daughter with Neurological Disease. Parasites \& Vectors 3, (2010).

144 Munana, K. R., Vitek, S. M., Hegarty, B. C., Kordick, D. L. \& Breitschwerdt, E. B. Infection of Fetal Feline Brain Cells in Culture with Bartonella henselae. Infection and Immunity 69, 564$569,(2001)$.

145 Wolters, M., Zobiak, B., Nauth, T. \& Aepfelbacher, M. Analysis of Yersinia enterocolitica Effector Translocation into Host Cells Using Beta-lactamase Effector Fusions. Journal of Visualized Experiments : JoVE, e53115, (2015).

146 Seenappanahalli Nanjundaiah, Y., Wright, D. A., Baydoun, A. R., O’Hare, W. T., Ali, Z., Khaled, Z. \& Sarker, M. H. Lactobacillus rhamnosus GG Conditioned Media Modulates Acute Reactive Oxygen Species and Nitric Oxide in J774 Murine Macrophages. Biochemistry and Biophysics Reports 6, 68-75, (2016).

147 Messaoudi, I., Estep, R., Robinson, B. \& Wong, S. W. Nonhuman Primate Models of Human Immunology. Antioxidants \& Redox Signaling 14, 261-273, (2011).

148 Karimova, G., Pidoux, J., Ullmann, A. \& Ladant, D. A Bacterial Two-hybrid System Based on a Reconstituted Signal Transduction Pathway. Proceedings of the National Academy of Sciences of the USA 95, 5752-5756, (1998).

149 Ham, T. S., Lee, S. K., Keasling, J. D. \& Arkin, A. P. A Tightly Regulated Inducible Expression System Utilizing the Fim Inversion Recombination Switch. Biotechnology and Bioengineering 94, (2006). 


\section{References}

150 Carroll, P., Brown, A. C., Hartridge, A. R. \& Parish, T. Expression of Mycobacterium tuberculosis Rv1991c Using an Arabinose-inducible Promoter Demonstrates its Role as a Toxin. FEMS Microbiology Letters 274, 73-82, (2007).

151 Franzoso, G., Carlson, L., Brown, K., Daucher, M. B., Bressler, P. \& Siebenlist, U. Activation of the Serum Response Factor by p65/NF-kappaB. The EMBO Journal 15, 3403-3412, (1996). 


\section{Appendix}

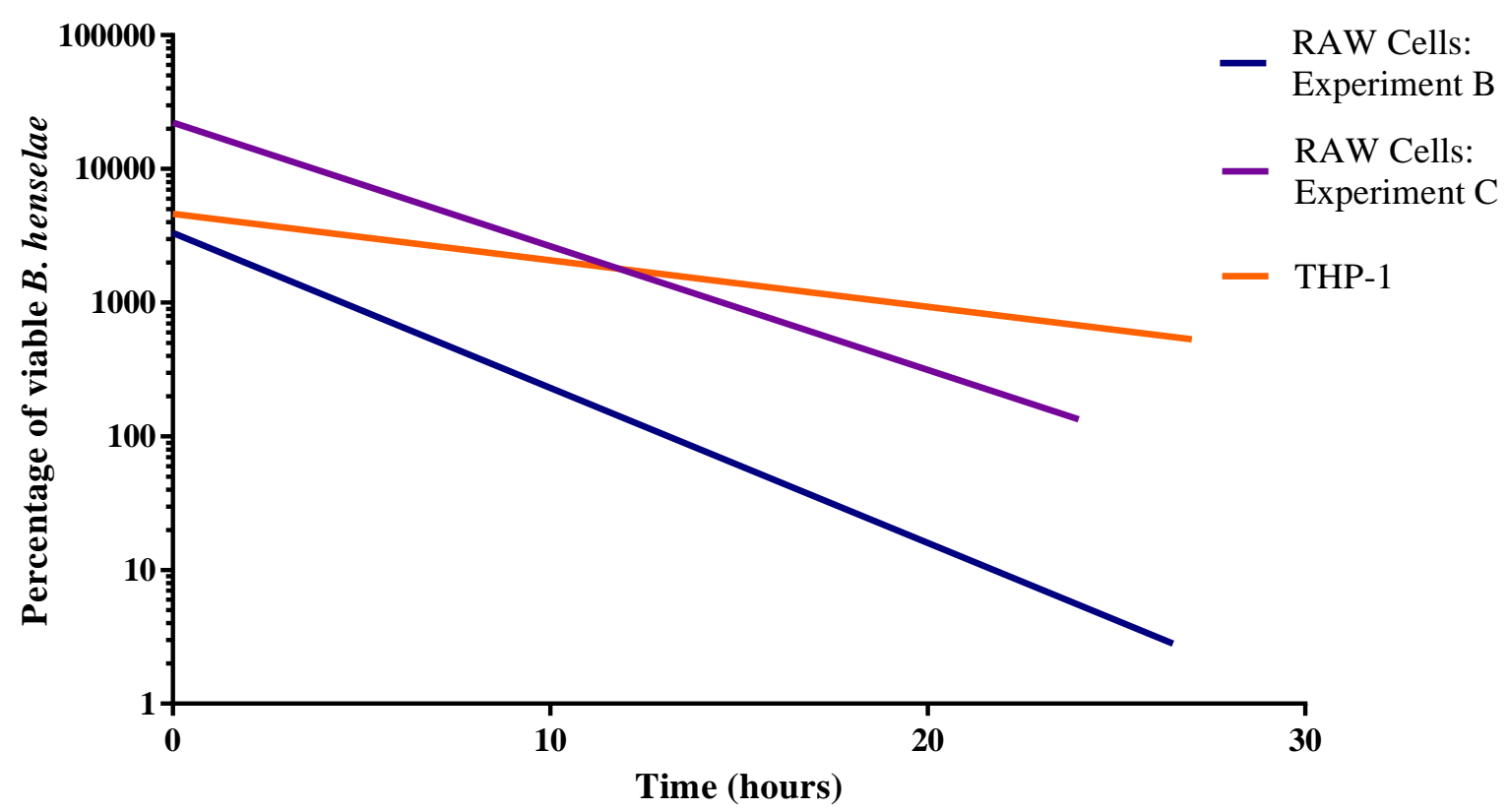

Appendix Figure 1: RAW 267.4 cell killing of intracellular B. henselae with a logarithmic y-axis. RAW 267.4 cells were infected with $B$. henselae, then extracellular $B$. henselae were killed by gentamicin treatment. Intracellular $B$. henselae were quantified over the hours following internalization, to determine the rate at which the bacteria could persist intracellularly. B. henselae killing appears faster in the two RAW 267.4 cell experiments, compared to infection of THP-1 cells, although statistical analysis cannot confirm this is significant due to low biological replicates. 
Appendix

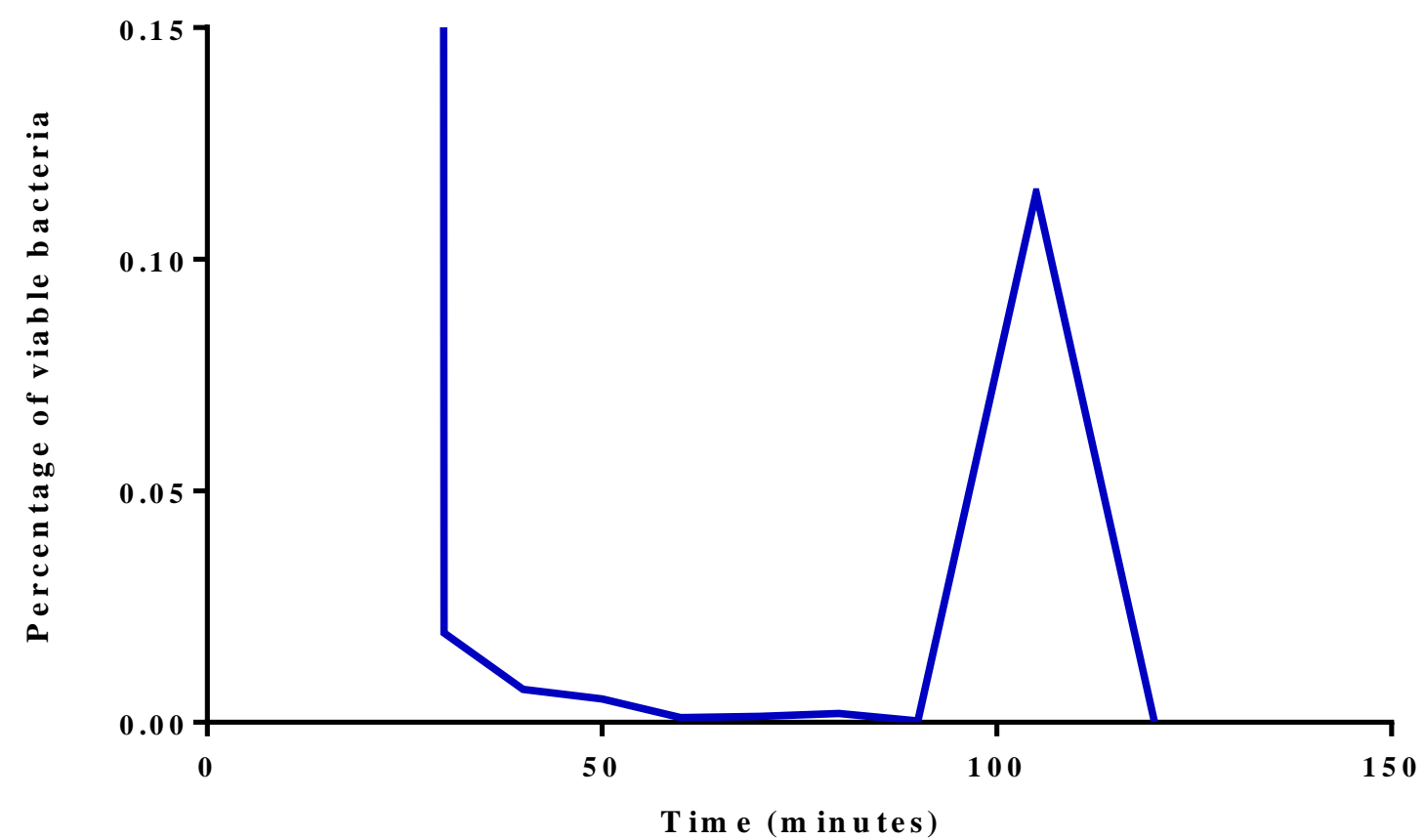

Appendix Figure 2: B. quintana recovered after gentamicin exposure, includes outlier. This figure shows the effective killing of B. quintana by the antibiotic gentamicin, with $99.999 \%$ of bacteria killed after one hour of treatment; after another hour no viable bacteria could be detected. An outlier can see seen at 105 minutes of gentamicin treatment which resulted in 320 visible colonies, indicating 3200 viable bacteria remained $(0.1143 \%$ of the total bacteria before gentamicin treatment). This was unexpectedly high and was possibly due to the formation of large bacterial aggregates that prevent gentamicin killing. However, viable bacteria were not detected thereafter. 
Appendix

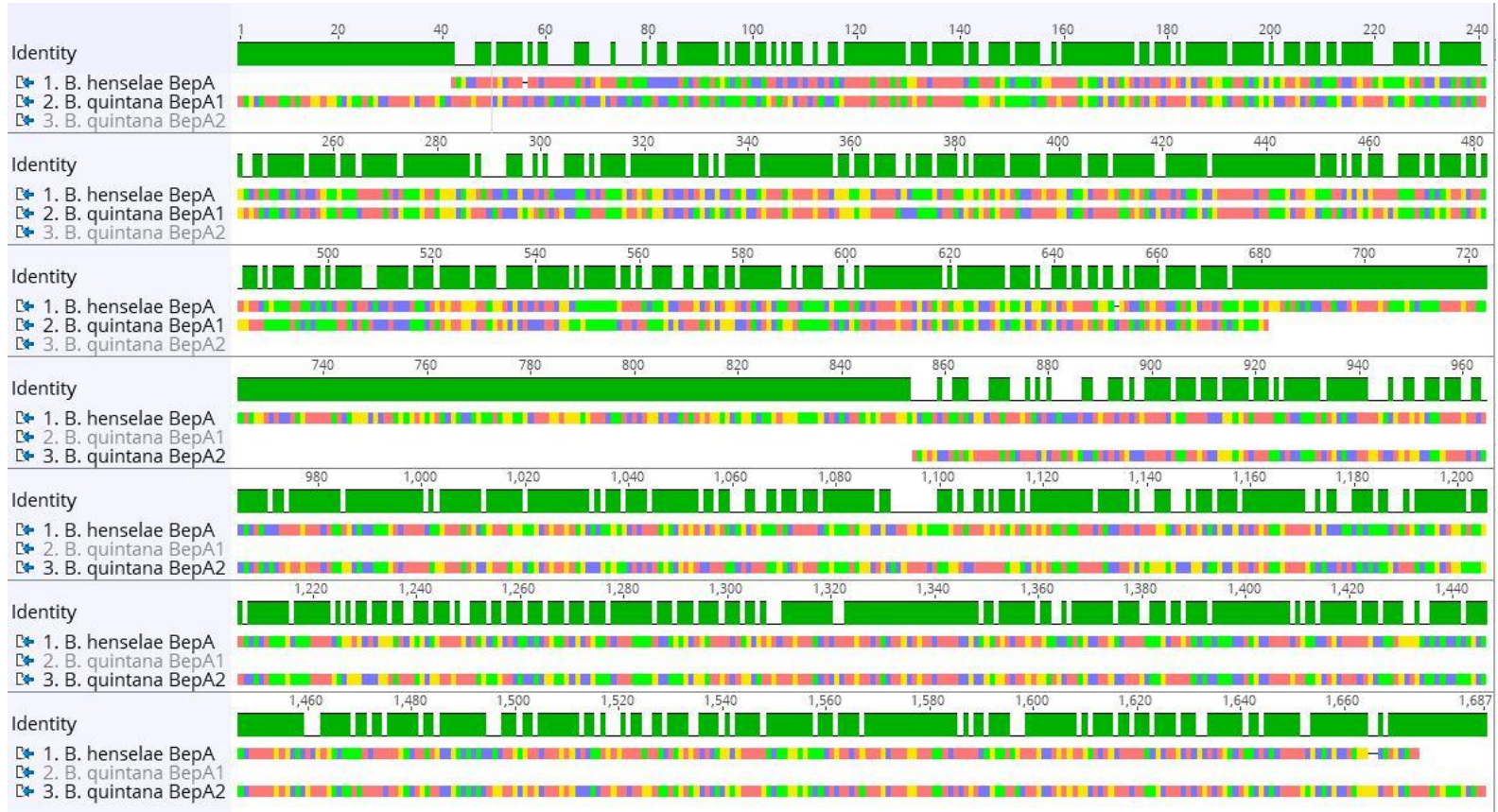

Appendix Figure 3: Homology between B. henselae BepA and B. quintana BepA1/2. When sequences of B. quintana BepA1 and BepA2 are compared to B. henselae BepA there is a 70\% sequence homology. Breaks in the green bar indicate areas of mismatch in amino acid sequence.

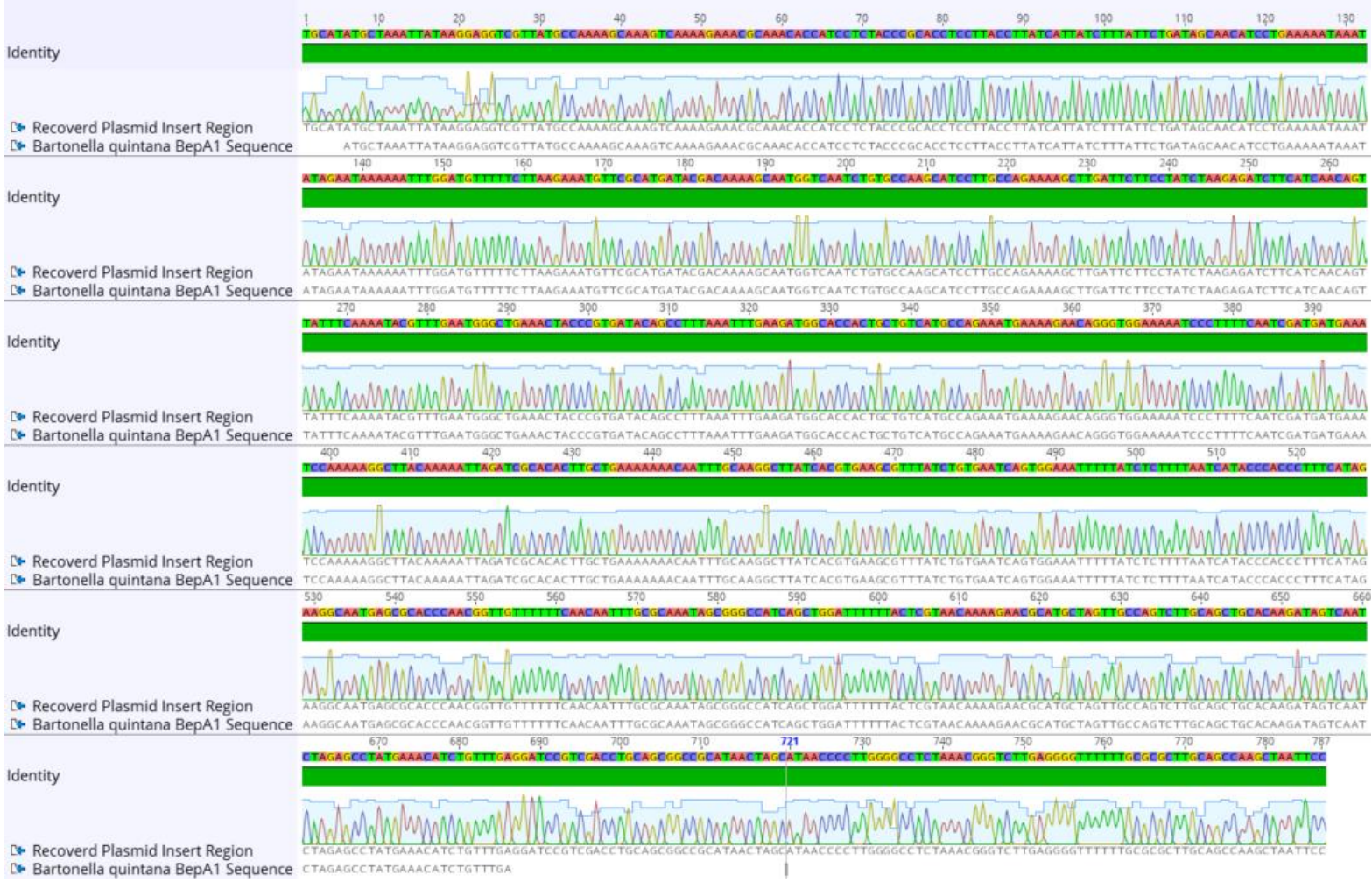

Appendix Figure 4: bepAl insert into pGBKT7. This figure shows the complete sequence identity (no breaks in the green bar) between the bepA1 from B. quintana JK31 genome and the sequence from pGBKT7 following cloning. This indicates there was no errors during PCR of the insert from the $B$. quintana genome and the insert was successfully inserted into pGBKT7. The insert is in frame and will therefore be translated into a functional BepA1 protein with the GAL4-BD translation fusion. 
Appendix

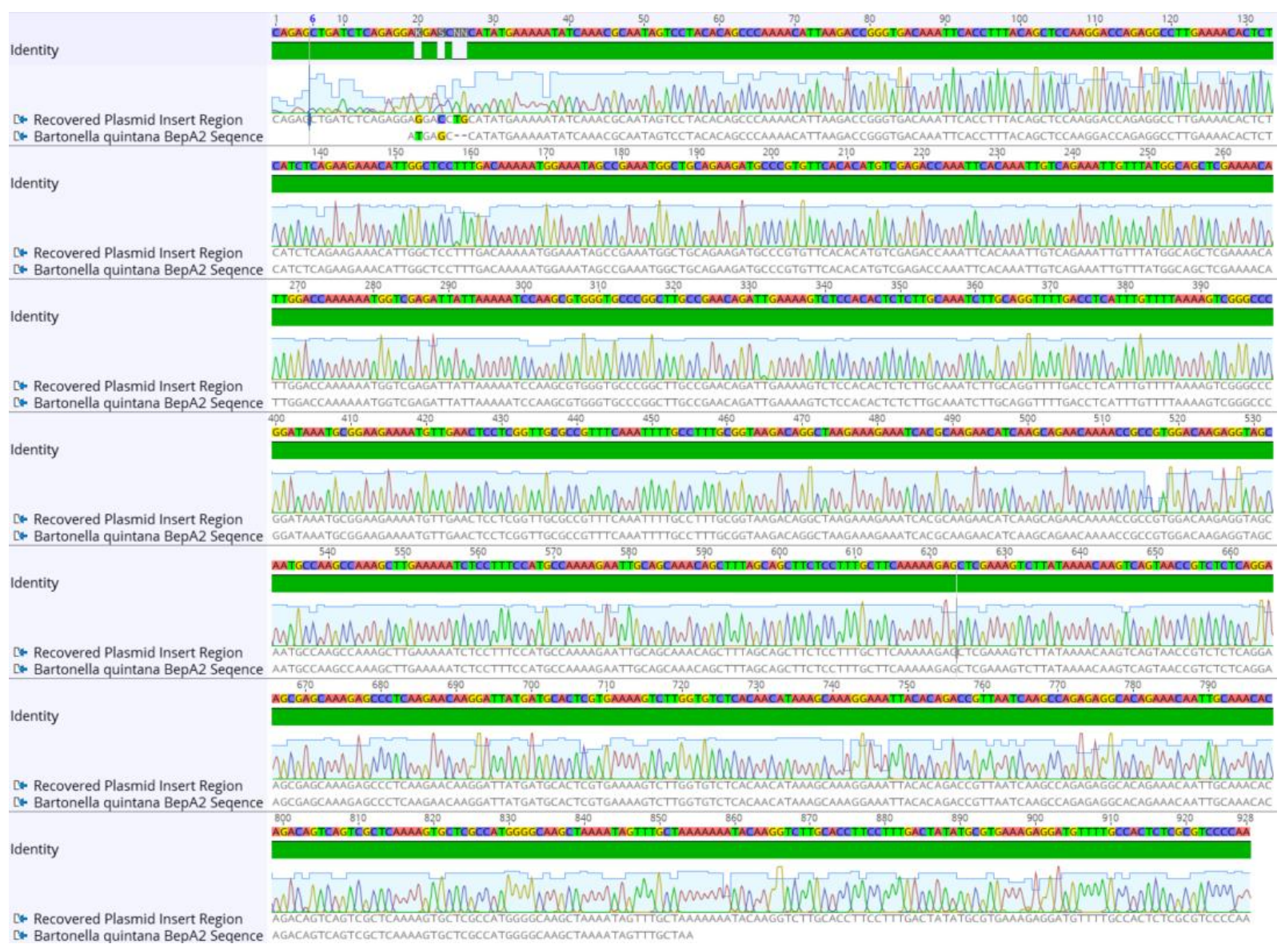

Appendix Figure 5: bepA2 insert in pGBKT7. This figure shows the incomplete sequence identity between the bepA2 from B. quintana JK31 genome and the sequence from pGBKT7 following cloning. This suggests the insert was altered following cloning, rendering the sequencing non-translatable. Although most of the recovered sequence matches the BepA2 sequence from the B. quintana JK31 genome, the beginning of the gene differs, with a mutation to the start codon and the addition of two nucleotides, rendering the gene non-translatable and out of frame. This recovered clone would not produce a function BepA2 protein with the GAL4-BD translational fusion. Breaks in the green bar indicate areas of mismatch in amino acid sequence.

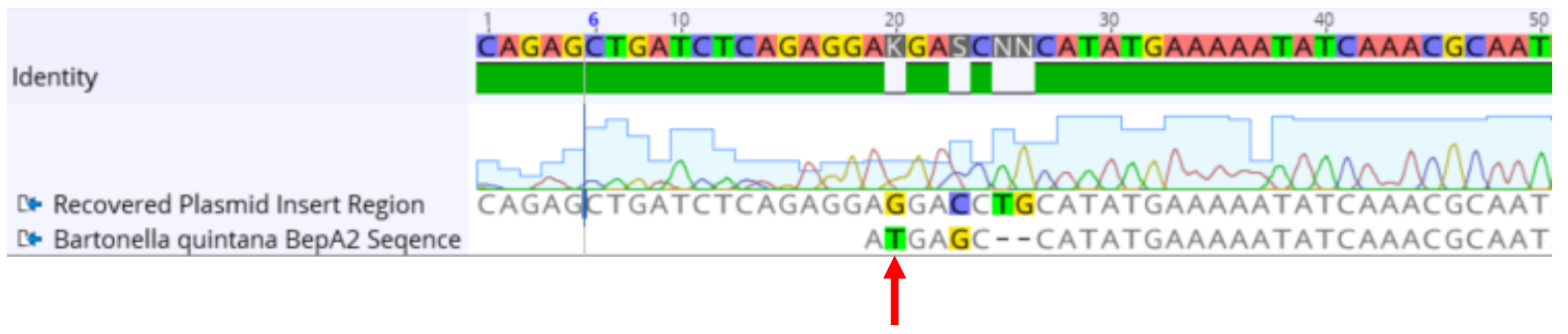

Appendix Figure 6: bepA2 in pGBKT7, Mutated Start Codon. This figure shows a magnified view showing of the mutated bases in the beginning of the bepA2 gene sequence recovered from pGBKT7, following cloning. An alteration of the start codon ATG to AGG, can be seen, noted with the red arrow. 
Appendix

Additionally, the addition of a $\mathrm{T}$ and $\mathrm{G}$ nucleotide shift the remaining gene out of frame. Together these mutations would prevent correct translation of the BepA2 protein with the GAL4-BD translational fusion. Breaks in the green bar indicate areas of mismatch in amino acid sequence.
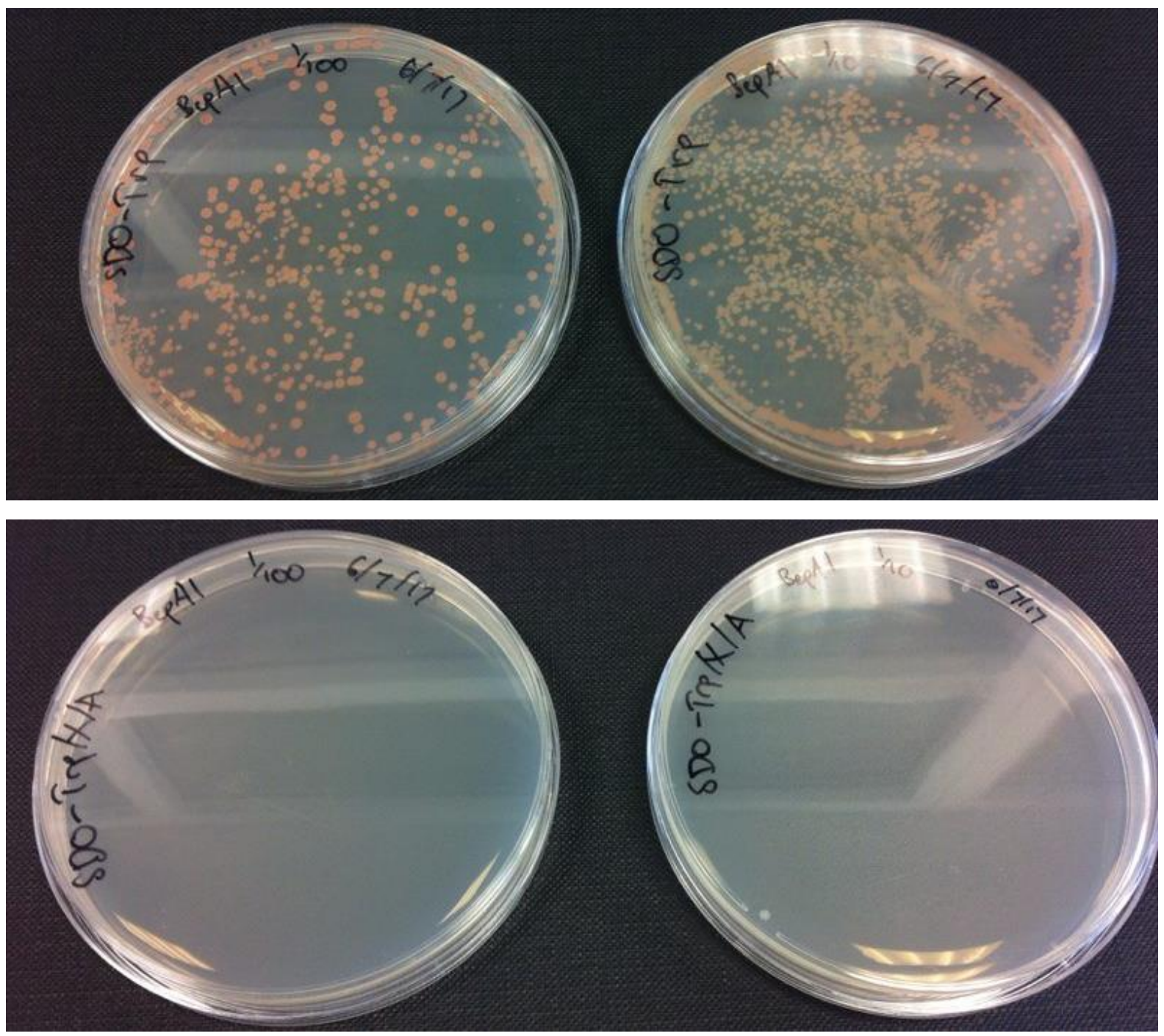

Appendix Figure 7: BepA1 auto-activation test. The auto-activation test ensures the bait protein (BepA1 in this case) does not activate the Y2HS reporters in the absence of an interacting partner. Y2HGold containing BepA1 grow successfully on single drop out plates (seen on the top two plates). The single drop out media only acts to maintain the bait plasmid, without the requirement of reporter activation for survival. Y2HGold containing BepA1 does not grow on plates with the antibiotic aureobasidin-A and $\mathrm{X}-\alpha-\mathrm{Gal}$ (no colonies can be seen on the bottom two plates). Survival in the presence of aureobasidinA results from reporter activation; lack of survival indicates BepA1 is not capable of activating the reporter genes without an interacting partner. 


\section{Appendix}

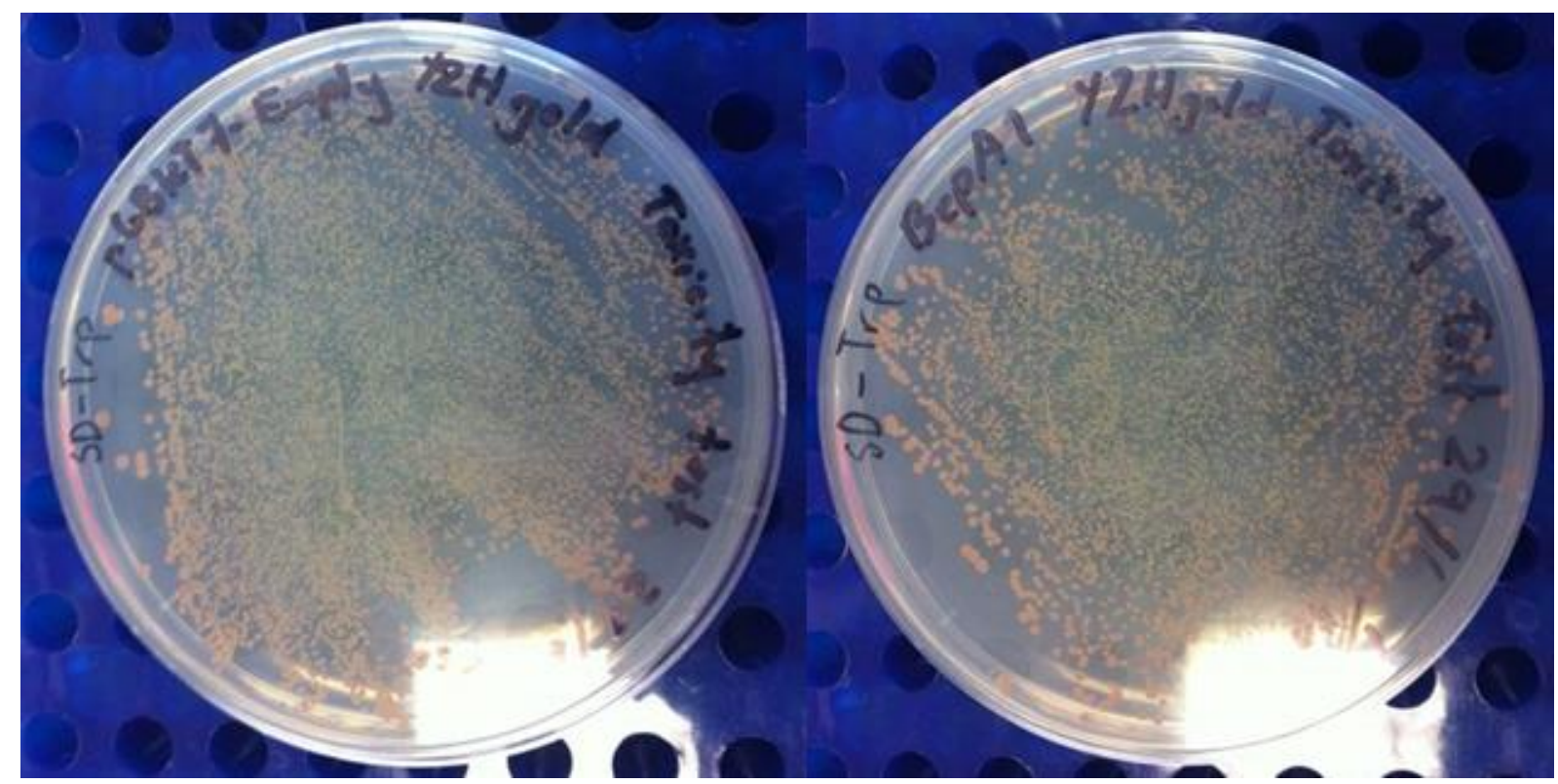

Appendix Figure 8: BepA1 toxicity test. The toxicity test ensures the BepA1 does not have a toxic effect on Y2HGold yeast, impairing growth.Y2HGold containing the empty pGBKT7 vector (seen on the left) grows equally well as pGBKT7 with BepA1 (seen on the right). Colonies are similar sizes on both plates indicating the presence of BepA1 within Y2HGold yeast does not have a toxic effect, reducing growth. 
Appendix

Homo sapiens HAS2 antisense RNA 1 (HAS2-AS1), antisense RNA

Sequence ID: NR_002835.2 Length: 2300 Number of Matches: 1

Range 1: 1294 to 2015 GenBank Graphics

Next Match A Previous Match

\begin{tabular}{lllll}
\hline Score & Expect & Identities & Gaps & Strand \\
1312 bits(710) & 0.0 & $718 / 722(99 \%)$ & $0 / 722(0 \%)$ & Plus/Plus \\
\hline
\end{tabular}

Query 50 GTACCTTATGGAACCCAAACCTGTTATGTTTTCACCTGTACAAGGCAACAGAGAAAACAT 109

sbjet 1294 GTACCTTATGGAACCCATACCTGTTATGTTTCACCTGTACAAGGCAACAGAGAAAACAT 1353

Query 110 GGTAGTTTTTATTTGAGTAGATGTGACTTAGCAATATGTTGTTGCTGACCTCCATCCCAC 169

Sbjet 1354 GGTAGTTTTAACTTGAGAGATGTGACTTAGCAATATGTTGTTGCTGACCTCCATCCCAC 1413

Query 170 AgCACAAAACATATGTTTATATGTCTGACAGACAAAGTATCCAGAACAAGGATTTAGTAC 229

Sbjet 1414 AGCACAAAACATATGTTTATATGTCTGACAGACAAAGTATCCAGAACAAGGATTTAGTAC 1473

Query 230 CTTCATAATCCTCTGTATATGTGGTTCTAGAACTTTTGGTAAAGAAACTCTTTACACTCT 289

Sbjet 1474 CTTCATAATCCTCTGTATATGTGGTCTAGACTTTGGTAAGAACTCTTATACTCT 1533

Query 290 TATTAATTATTGAgACCTCaaaaaagtTTATATgTTATATGTATCAATATTTACCACA 349

Sbjet 1534 TATTAATTATTGAGACCTCAAAAAAGTTATATGTATATGTATCAATATTTACCACA 1593

Query 350 TTAGACATTAAAGCTGACTTAAAAAGTTAAAATACTTAAGTCATTTGAAGTAATAATAAA 409

Sbjet 1594 TTAGACATTAAAGCTGACTTAAAAAGTAAAATACTTAAGTCATTGAAGTAATAATAAA 1653

Query 410 TGCAGTTACATACCAACTTAATAACATTTTGCGAAAATAGCTATTTTTCAAAACCTAAAG 469

sbjet 1654 TGCAGTTACATACCAACTTAATAACATTTTGCGAAAATAGCTATTTTTCAAAACCTAAAG 1713

Query 470 TGAGAAAAGTGGCAGTTTTCCATTTTTGCAAATCTCTTTGCTGTCTAGCTGATAGAAGAC 529

Sbjct 1714 TGAGAAAAGTGCAGTTTCCATTTTGCAATCTCTTGCTGTCTAGCTGATAGAAGAC 1773

Query 530 AGCTGGATTCTCCTATTTGCTTTTGCATTCAATTGTTGTTTTGATTGAAGTATATGaaaa 589

Sbjct 1774 AGCTGGATTCTCCTATTGCTTTTGCATTCAATTGTTGTTTTGATTGAAGTATATGAAAA 1833

Query 590 aaaaTCTGGTGTCATACACATATATAATTGGGAATGGAAGAGTATTTACATAGGCTTTT 649

sbjet 1834 AAAATCTGGTGTCATACACATATATAATTGGGAAATGAAGAGTATTTACACAGGCTTTT 1893

Query 650 TTAGATATTCTTCCTTTATACTACATCAAAACTTGATAAGTTGCAATGTTGTGAATTGGC 709

sbjet 1894 TTAGATATTCTTCCTTATACTACATCAAACTTGATAAGTTGCAATGTTGTGAATGGC 1953

Query 710 CCAGGGCTTGGCCCTCTGAAGATTTGCTGAAAATCACTGACATGAAGTAGATTAATAGGA 769

sbjet 1954 CCAGGGCTTGGCCTCTGAAGATTTGCTGAAAATCACTGACATGAAGTAGATTAATAGGA 2013

Query $770 \quad$ TA 771

Sbjct 2014 TA 2015

Appendix Figure 9: BLAST match of Y2HS prey sequence - HAS2 antisense RNA 1 \#1. This figure shows the $99 \%$ identity between the sequence recovered from a library plasmid during the Y2HS assay and the HAS2 antisense RNA 1 sequence. This suggests there was an independent interaction between the BepA1 bait protein and the prey protein translated from this sequence, during Y2HS assay. 
Appendix

Homo sapiens HAS2 antisense RNA 1 (HAS2-AS1), antisense RNA

Sequence ID: NR_002835.2 Length: 2300 Number of Matches: 1

Range 1: 1294 to 2051 GenBank Graphics

$\nabla$ Next Match A Previous Match

\begin{tabular}{lllll}
\hline Score & Expect & Identities & Gaps & Strand \\
1375 bits(744) & 0.0 & $753 / 758(99 \%)$ & $0 / 758(0 \%)$ & Plus/Plus \\
\hline
\end{tabular}

Query 60 GTACCTTATGGAACCCAAACCTGTTATGTTTTCACCTGTACAAGGCAACAGAGAAAACAT 119

Sbjct 1294 GTACCTTATGGAACCCATACCTGTTATGTTTTCACCTGTACAAGGCAACAGAGAAAACAT 1353

Query 120 GGTAGTTTTATTTGAGTAGATGTGACTTAGCAATATGTTGTTGCTGACCTCCATCCCAC 179

Sbjct 1354 GGTAGTTTTTACTTGAGTAGATGTGACTTAGCAATATGTTGTTGCTGACCTCCATCCCAC 1413

Query 180 AgCACAAACATATGTTTATATGTCTGACAGACAAAGTATCCAGAACAAGGATTTAGTAC 239

sbjet 1414 AGCACAAAACATATGTTTATATGTCTGACAGACAAAGTATCCAGAACAAGGATTTAGTAC 1473

Query 240 CTTCATAATCCTCTGTATATGTGGTTCTAGAACTTTTGGTAAAGAACTCTTTACACTCT 299

Sbjet 1474 CTTCATAATCCTCTGTATATGTGGTCTAGAACTTTGGTAAAGAACTCTTTATACTCT 1533

Query 300 TATTAATTATTGAgAaCctCaaaaaagtTTATATGTTATATGTATCAATATTTACCACA 359

sbjct 1534 TATTAATTATTGAGACCTCAAAAAAGTTTATATGTTATATGTATCAATATTTACCACA 1593

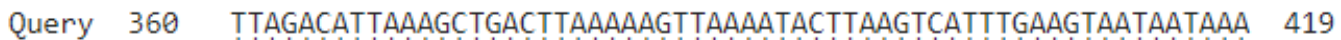

Sbjct 1594 TTAGACATTAAAGCTGACTTAAAAAGTTAAAATACTTAAGTCATTTGAAGTAATAATAAA 1653

Query 420 TGCAGTTACATACCAACTTAATAACATTTTGCGAAAATAGCTATTTTTCAAAACCTAAAG 479

Sbjct 1654 TGCAGTACATACCAACTTAATAACATTTTGCGAAAATAGCTATTTTTCAAAACCTAAAG 1713

Query 480 TGAGAAAAGTGGCAGTTTTCCATTTTTGCAAATCTCTTTGCTGTCTAGCTGATAGAAGAC 539

Sbjet 1714 TGAGAAAAGTGGCAGTTTCCATTTTGCAATCTCTTGCTGTCTAGCTGATAGAAGAC 1773

Query 540 AGCTGGATTCTCCTATTTGCTTTTGCATTCAATTGTTGTTTTGATTGAAGTATATGaaaa 599

sbjct 1774 AGCTGGATCTCCTATTGGTTTGCATCAATTGTGTTTGATTGAAGTATATGAAA 1833

Query 600 aaaaTCTGGTGTCATACACATATATNATTGGGAATGGAGGGTATTTACATAGGCTTTT 659

sbjct 1834 AAAATCTGGTGTCATACACATATATAATTGGGAAATGGAAGAGTATTTACACAGGCTTTT 1893

Query 660 TTAGATATTCTTCCTTTATACTACATCAAAACTTGATAAGTTGCAATGTTGTGAATTGGC 719

sbjet 1894 TTAGATATTCTTCCTTATACTACATCAAAACTTGATAAGTTGCAATGTTGTGAATTGGC 1953

Query 720 CCAGGGCTTGGCCCTCTGAAGATTTGCTGAAAATCACTGACATGAAGTAGATTAATAGGA 779

sbjet 1954 CCAGGGCTTGGCCCTCTGAAGATTTGCTGAAAATCACTGACATGAAGTAGATTAATAGGA 2013

Query 780 TAAAAGGCATATACATTTATTTAATGTGTATACACAGC 817

sbjct 2014 TAAAAGGCATATACATTTATTTAATGTGTATACACAGC 2051

Appendix Figure 10: BLAST match of Y2HS prey sequence - HAS2 antisense RNA 1 \#2. Refer to Appendix Figure 9 for a description of this figure. 
Appendix

Homo sapiens HAS2 antisense RNA 1 (HAS2-AS1), antisense RNA

Sequence ID: NR_002835.2 Length: 2300 Number of Matches: 1

Range 1: 1294 to 2093 GenBank Graphics

$\nabla$ Next Match A Previous Match

\begin{tabular}{lllll}
\hline Score & Expect & Identities & Gaps & Strand \\
1456 bits $(788)$ & 0.0 & $796 / 800(99 \%)$ & $0 / 800(0 \%)$ & Plus/Plus \\
\hline
\end{tabular}

Query 59 GTACCTTATGGAACCCAAACCTGTTATGTTTTCACCTGTACAAGGCAACAGAGAAAACAT 118

sbjet 1294 GTACCTTATGGAACCCATACCTGTTATGTTTCACCTGTACAAGGCAACAGAGAAAACAT 1353

Query 119 GGTAGTTTTTATTTGAGTAGATGTGACTTAGCAATATGTTGTTGCTGACCTCCATCCCAC 178

Sbjet 1354 GGTAGTTTTAACTTGAGTAGATGTGACTTAGCAATATGTTGTTGCTGACCTCCATCCCAC 1413

Query 179 AgCACAAAACATATGTTTATATGTCTGACAGACAAAGTATCCAGAACAAGGATTTAGTAC 238

Sbjet 1414 AGCACAAAACATATGTTTATATGTCTGACAGACAAAGTATCCAGAACAAGGATTTAGTAC 1473

Query 239 CTTCATAATCCTCTGTATATGTGGTTCTAGAACTTTTGGTAAAGAAACTCTTTACACTCT 298

Sbjet 1474 CTTCATAATCCTCTGTATATGTGGTCTAGACTTTGGTAAAGAACTCTTATACTCT 1533

Query 299 TATTAATTATTGAGACCTCaaaaaagtTTATATGTTATATGTATCAATATTTACCACA 358

Sbjet 1534 TATTAATTATTGAGACCTCAAAAAAGTTATATGTATATGTATCAATATTTACCACA 1593

Query 359 TTAGACATTAAAGCTGACTTAAAAAGTTAAAATACTTAAGTCATTTGAAGTAATAATAAA 418

Sbjct 1594 TTAGACATTAAAGCTGACTTA| || || 1653

Query 419 TgCAGTTACATACCAACTTAATAACATTTTGCGAAAATAGCTATTTTTCAAAACCTAAAG 478

Sbjet 1654 TGCAGTTACATACCAACTTAATAACATTTTGCGAAAATAGCTATTTTTCAAAACCTAAAG 1713

Query 479 TGAGAAAAGTGGCAGTTTTCCATTTTTGCAAATCTCTTTGCTGTCTAGCTGATAGAAGAC 538

Sbjct 1714 TGAGAAAAGTGCAGTTTCCATTTTGCAATCTCTTGCTGTCTAGCTGATAGAAGAC 1773

Query 539 AGCTGGATTCTCCTATTTGCTTTTGCATTCAATTGTTGTTTTGATTGAAGTATATGaaaa 598

sbjet 1774 AGCTGGATTCTCCTATTTGCTTTTGCATTCAATTGTTGTTTGATTGAAGTATATGAAAA 1833

Query 599 aaaaTCTGGTGTCATACACATATATAATTGGGAATGGAAGAGTATTTACATAGGCTTTT 658

Sbjct 1834 AAAATCTGGTGTCATACACATATATAATTGGGAAATGGAAGAGTATTTACACAGGCTTTT 1893

Query 659 TTAGATATTCTTCCTTTATACTACATCAAAACTTGATAAGTTGCAATGTTGTGAATTGGC 718

sbjct 1894 TTAGATATTCTTCCTTATACTACATCAAAACTTGATAAGTTGCAATGTTGTGAATTGG 1953

Query 719 CCAGGGCTTGGCCCTCTGAAGATTTGCTGAAAATCACTGACATGAAGTAGATTAATAGGA 778

sbjet 1954 CCAGGGCTTGCCCTCTGAAGATTTGCTGAAATCACTGACATGAAGTAGATTAATAGGA 2013

Query 779 TAAAAGGCATATACATTTATTTAATGTGTATACACAGCGGCCTTTAGAATGAAGACCCAA 838

sbjet 2014 TAAAAGGCATATACATTATTTAATGGTATACACAGCGGCCTTTAGAATGAaGACCCAA 2073

Query 839 AGATACAGAAGAATTGTCT 858

Sbjct 2074 AGATACAGAAGAAATTGTCT 2093

Appendix Figure 11: BLAST match of Y2HS prey sequence - HAS2 antisense RNA 1 \#3. Refer to Appendix Figure 9 for a description of this figure. 
Appendix

Homo sapiens HAS2 antisense RNA 1 (HAS2-AS1), antisense RNA Sequence ID: NR_002835.2 Length: 2300 Number of Matches: 1

Range 1: 1294 to 1943 GenBank Graphics V Next Match A Previous Match

Score Expect Identities Strand 1179 bits(638) 0.0 $646 / 650(99 \%)$ Gaps Plus/Plus

Query 44 GTACCTTATGGAACCCAAACCTGTTATGTTTTCACCTGTACAAGGCAACAGAGAAAACAT 103

sbjet 1294 GTACCTTATGGAACCCATACCTGTTATGTTTTCACCTGTACAAGGCAACAGAGAAAACAT 1353

Query 104 GGTAGTTTTTATTTGAGTAGATGTGACTTAGCAATATGTTGTTGCTGACCTCCATCCCAC 163

sbjet 1354 GGTAGTTTTTACTTGAGTAGATGTGACTTAGCAATATGTTGTTGCTGACCTCCATCCCAC 1413

Query 164 AgCACAAAACATATgTTTATATgTCTGACAGACAAAgTATCCAGAACAAGGATTTAGTAC 223

Sbjet 1414 AGCACAAAACATATGTTATATGTCTGACAGACAAAGTATCCAGAACAAGGATTAGTAC 1473

Query 224 CTTCATAATCCTCTGTATATGTGGTTCTAGAACTTTTGGTAAAGAAACTCTTTACACTCT 283

Sbjet 1474 CTTCATAATCCTCTGTATATGTGGTCTAGACTTTGGTAAAGAACTCTTATACTCT 1533

Query 284 TATTAATTATTGAGACCTCaaaaaaaGTTTATATGTTATATGTATCAATATTTACCACA 343

sbjet 1534 TATTAATTATTGAGAACCTCAAAAAAAGTTATATGTTATATGTATCAATATTTACCACA 1593

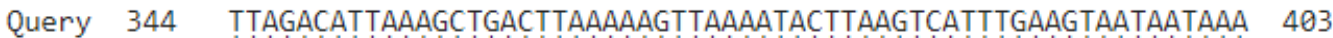

sbjet 1594 TTAGACATTAAAGCTGACTTAAAAAGTTAAAATACTTAAGTCATTTGAGGTAATAATAAA 1653

Query 404 TGCAGTTACATACCAACTTAATAACATTTTGCGAAAATAGCTATTTTTCAAAACCTAAAG 463

Sbjet 1654 TGCAGTTACATACCAACTTAATAACATTTGCGAAATAGCTATTTTCAAAACCTAAAG 1713

Query 464 TGAGAAAAGTGGCAGTTTTCCATTTTTGCAAATCTCTTTGCTGTCTAGCTGATAGAAGAC 523

sbjet 1714 TGAGAAAAGTGGCAGTTTTCCATTTTTGCAAATCTCTTTGCTGTCTAGCTGATAGAAGAC 1773

Query 524 AGCTGGATTCTCCTATTTGCTTTTGCATTCAATTGTTGTTTTGATTGAAGTATATGaaaa 583

sbjet 1774 AGCTGGATTCTCCTATTTGCTTTTGCATTCAATTGTTGTTTTGATTGAAGTATATGAAAA 1833

Query 584 aaaaTCTGGTGTCATACACATATATAATTGGGAAATGGAAGAGTATTTACATAGGCTTTT 643

sbjet 1834 AAAATCTGGTGTCATACACATATATAATTGGGAAATGGAAGAGTATTTACACAGGCTTT 1893

Query 644 TTAGATATTCTTCCTTTATACTACATCAAAACTTGATAAGTTGCAATGTT 693

sbjct 1894 TTAGATATTCTTCCTTTATACTACATCAAAACTTGATAAGTTGCAATGTT 1943

Appendix Figure 12: BLAST match of Y2HS prey sequence - HAS2 antisense RNA 1 \#4. Refer to Appendix Figure 9 for a description of this figure. 
Appendix

Homo sapiens myocardial zonula adherens protein (MYZAP), transcript variant 1, mRNA Sequence ID: NM_001018100.4 Length: 2408 Number of Matches: 1

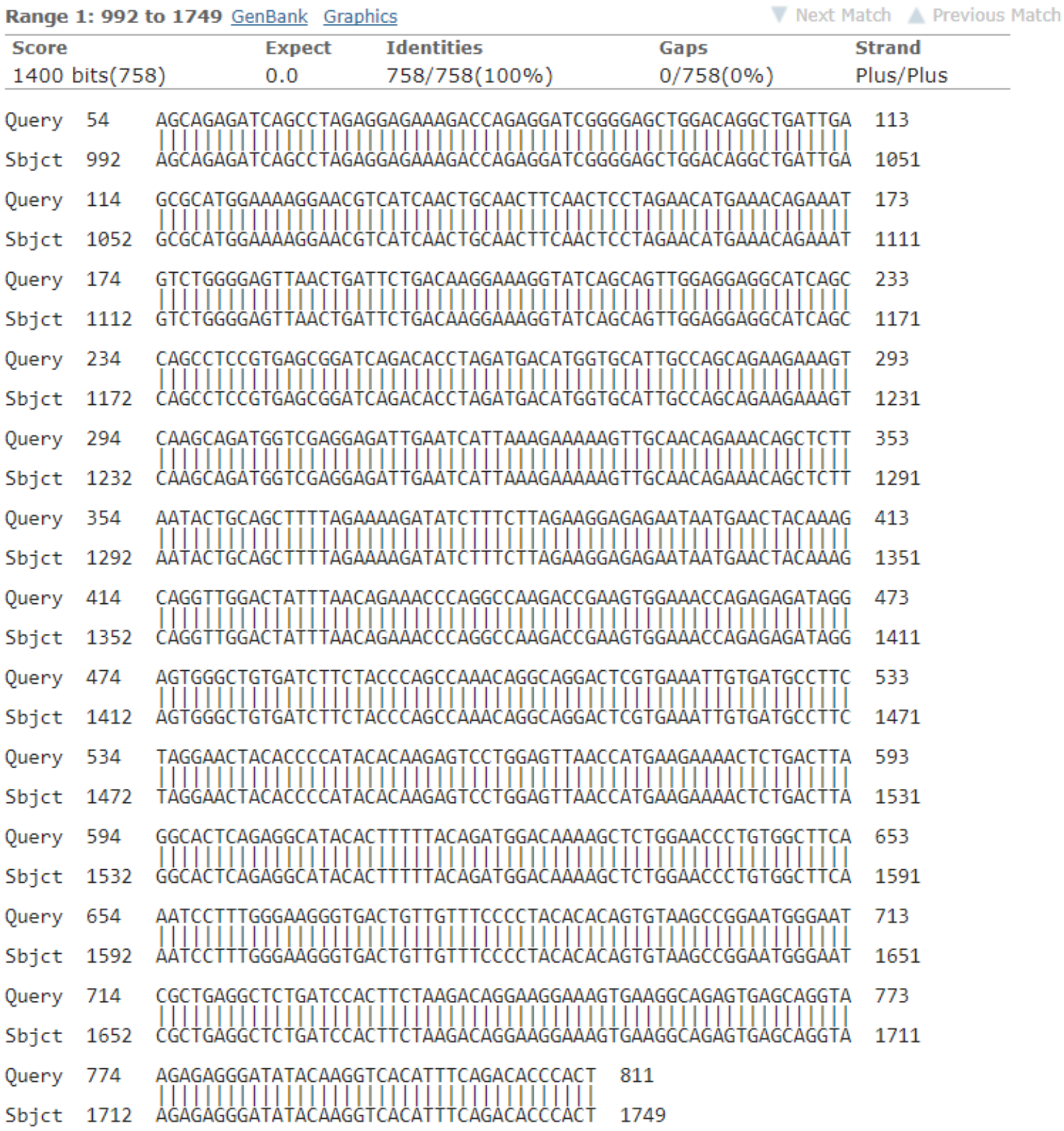

Appendix Figure 13: BLAST match of Y2HS prey sequence - Myozap protein \#1. This figure shows the $100 \%$ identity between the sequence recovered from a library plasmid during the Y2HS assay and the Myozap protein. This suggests there was an independent interaction between the BepAl bait protein and the prey Myozap protein, during Y2HS assay. 
Appendix

Homo sapiens myocardial zonula adherens protein (MYZAP), transcript variant 1, mRNA Sequence ID: NM_001018100.4 Length: 2408 Number of Matches: 1

Range 1: 992 to 1676 GenBank Graphics $\nabla$ Next Match A Previous Match

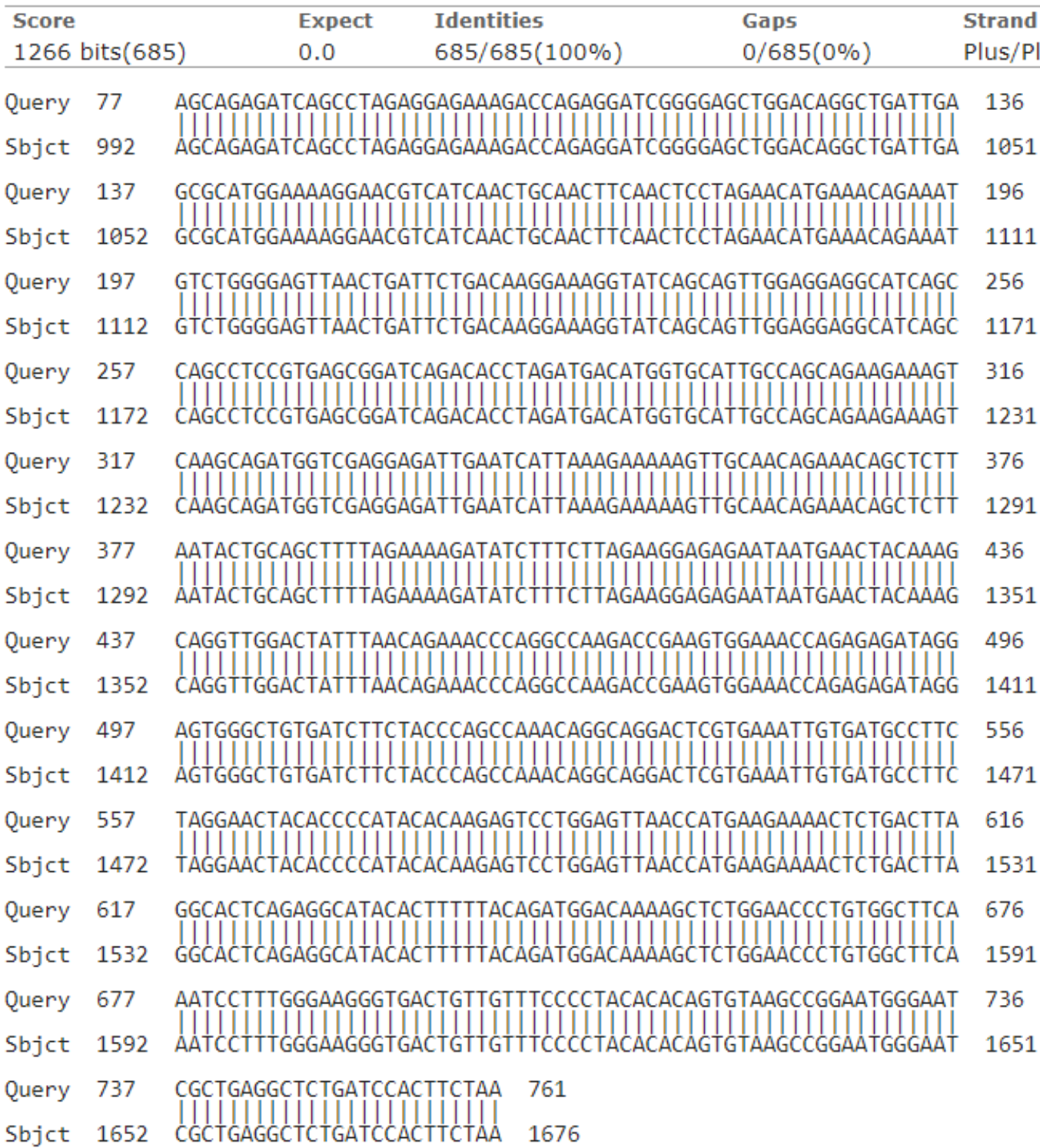

Appendix Figure 14: BLAST match of Y2HS prey sequence - Myozap protein \#2. Refer to Appendix Figure 13 for a description of this figure. 